\title{
CLINICAL AND FUNCTIONAL IMPACT OF COGNITIVE FLUCTUATIONS IN DEMENTIA
}

\author{
by \\ Brian Mainland \\ Master of Arts, Ryerson University, June 2011 \\ Bachelor of Science, Wilfrid Laurier University, June 2007
}

\author{
A dissertation \\ presented to Ryerson University \\ in partial fulfillment of the \\ requirements for the degree of \\ Doctorate of Philosophy \\ in the Program of \\ Psychology
}

Toronto, Ontario, Canada, 2015

(C) Brian Mainland 2015 


\section{Author's Declaration for Electronic Submission of a Dissertation}

I hereby declare that I am the sole author of this dissertation. This is a true copy of the dissertation, including any required final revisions, as accepted by my examiners.

I authorize Ryerson University to lend this dissertation to other institutions or individuals for the purpose of scholarly research.

I further authorize Ryerson University to reproduce this dissertation by photocopying or by other means, in total or in part, at the request of other institutions or individuals for the purpose of scholarly research.

I understand that my dissertation may be made electronically available to the public. 


\title{
Clinical and Functional Impact of Cognitive Fluctuations in Dementia \\ Doctorate of Philosophy 2015
}

\section{Brian Mainland}

\section{Psychology Program}

\section{Ryerson University}

\begin{abstract}
Cognitive fluctuations (CFs) are defined as spontaneous alterations in cognition, attention, and arousal, and are highly prevalent and disabling among people with dementia. CFs occur with a frequency of $80-90 \%$ in dementia with Lewy bodies (DLB), $40 \%$ in vascular dementia (VaD), and 20\% in Alzheimer's disease (AD). While CFs have been recognized as an important component of dementia, the majority of studies examining them have lacked objective methods of assessing their presence and severity, making it difficult to determine the degree of interference with other clinical features that can be attributable to fluctuations. The present study examined the nature and frequency of CFs in 55 individuals with dementia living in a long-term care facility. Participants underwent neuropsychological assessment to profile their current cognitive functioning. The Dementia Cognitive Fluctuation Scale (DCFS) was used to characterize CFs in this sample. Patients also completed brief cognitive measures on three separate occasions during a one-week period to obtain objective evidence of variability in cognitive performance. This study also assessed the association between CFs and informantbased measures of patients' quality of life, activities of daily living, and formal caregiver burden. Longitudinal cognitive data was analyzed retrospectively to determine patients' rate of cognitive decline over the past six months. Consistent with the limited research already completed in this area, this study found that increasing severity of CFs predicts lower cognitive performance and
\end{abstract}


reduced ability to complete activities of daily living. Also, this is the first study to demonstrate that CFs predict patients' overall quality of life and the degree of caregiver burden in primary nursing staff. Results of the current study suggest that CFs exert a broad range of influence over patients' functional abilities and wellbeing. Identifying which patients experience CFs could play an important role in developing individualized treatment plans best suited for patients specific care needs. 


\section{Acknowledgements}

First and foremost, I would like to thank my supervisor, Dr. Tisha Ornstein, for her continuing support and guidance throughout my graduate training. She has provided me with excellent guidance and ample opportunities throughout my development as a scientist practitioner. I would also like to thank Dr. Alexandra Fiocco, who provided support and feedback that has helped to strengthen this project. In addition, I would like to thank Dr. Nathan Herrmann and Dr. Kenneth Shulman, who provided guidance during my project development and assisted in securing access to study participants. I would like to thank Dr. Gwen Li Sin for her assistance with data collection. I would like to thank my classmates for their encouragement and advice, as well as for their cooperative collegial nature, which created memories that will last a lifetime. I would like to thank the patients who participated in this study, as well as primary nurses who graciously donated their time to complete study measures. Finally, I would like to thank my wife, family, and friends. Without your care, support and encouragement, none of this would have been possible. 
Dedication

To my mom and dad 


\section{Table of Contents}

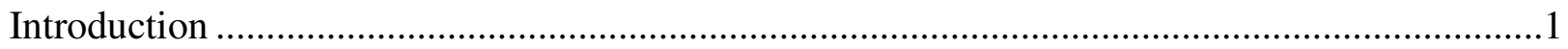

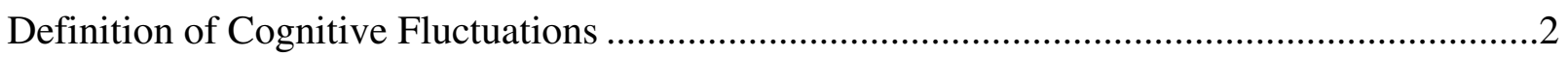

The Assessment of Cognitive Fluctuations ..................................................................

Clinician Assessment of Fluctuations (CAF) ............................................................

One Day Fluctuation Assessment (ODFAS) ...............................................................

Mayo Composite Fluctuations Scale (MCFS) ............................................................6

The Dementia Cognitive Fluctuation Scale (DCFS) ………………..........................

Characteristics of Patients With Cognitive Fluctuations ............................................................

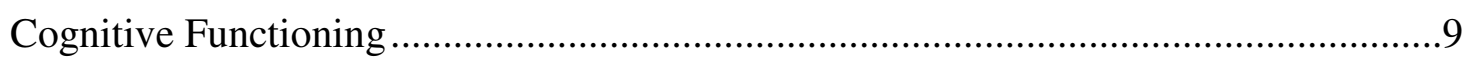

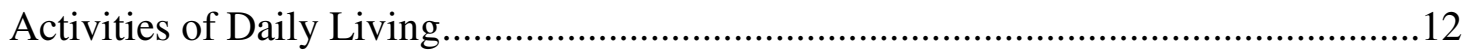

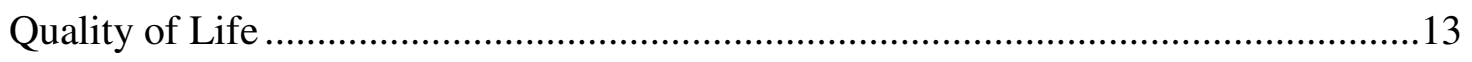

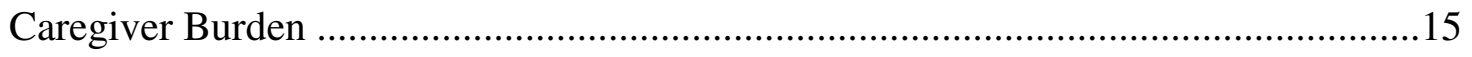

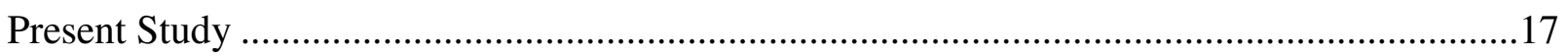

Study Aims and Hypotheses................................................................................. 18

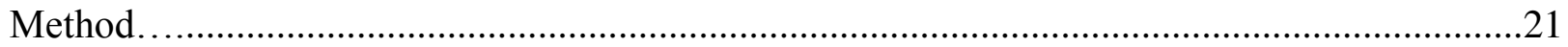

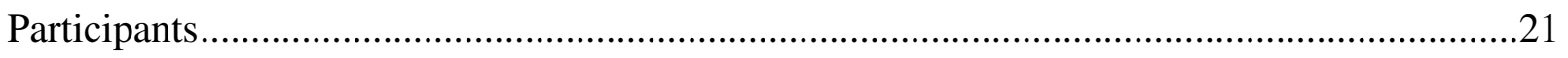

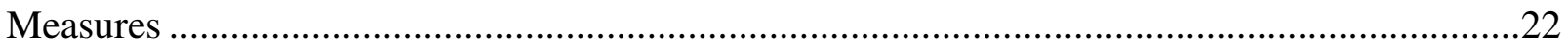

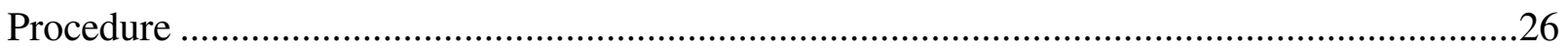

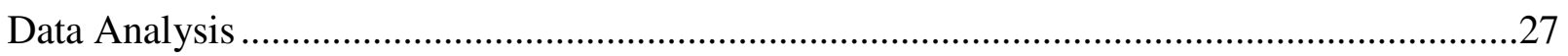

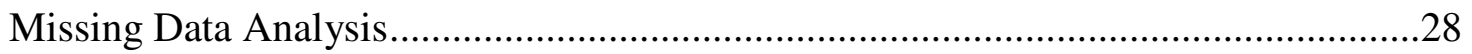

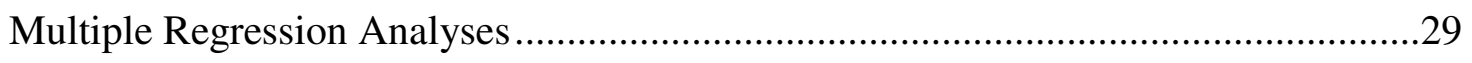

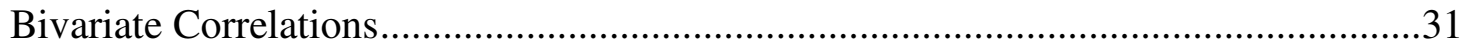


Results.

Descriptive Statistics.

Study Aim \#1a: Predicting Cognitive Test Performance.....................................................38

Study Aim \#1b: Cognitive Fluctuations and Variability in Cognitive Test Performance ..........44

Study Aim \#2a: Predicting Basic Activities of Daily Living .............................................46

Study Aim \#2b: Predicting Instrumental Activities of Daily Living ...................................50

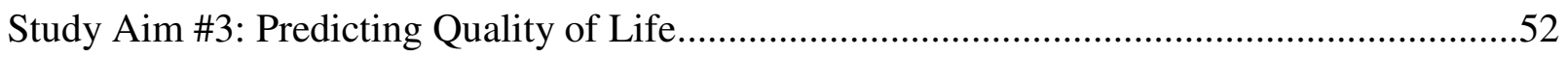

Study Aim \#4a: Predicting Primary Nurses’ Caregiver Burden Attitude Scores ....................56

Study Aim \#4b: Predicting Primary Nurses’ Caregiver Burden Strain Scores ......................62

Association Between Cognitive Test Variability and Other Study Measures .........................65

Post Hoc Analysis of Correlations Between Psychotropic Medications, Medical Comorbidity

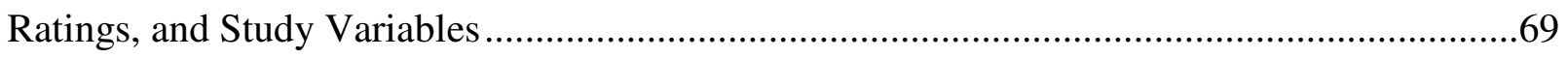

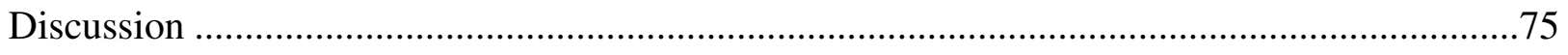

Cognitive Fluctuations and Level of Cognitive Functioning ...........................................75

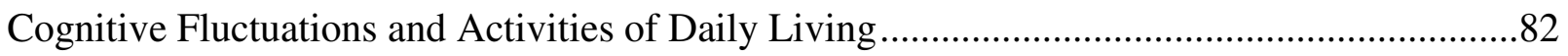

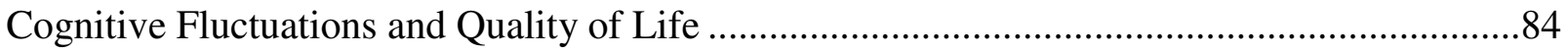

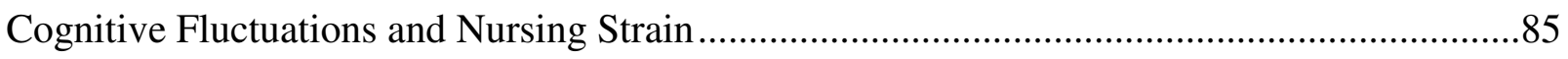

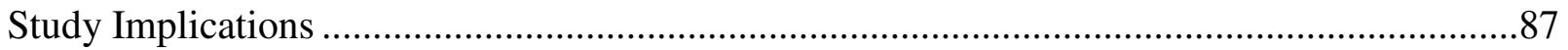

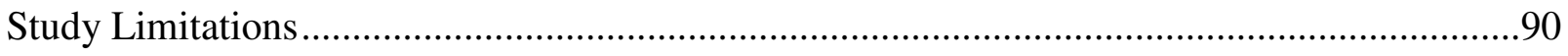

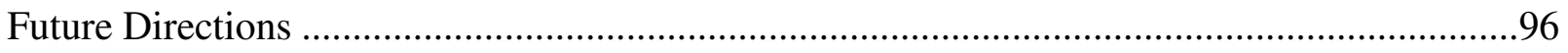

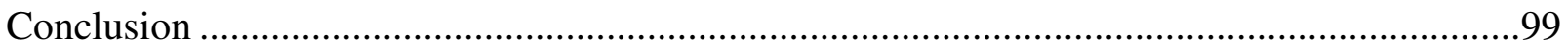

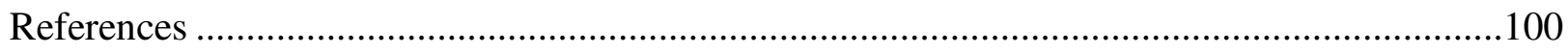




\section{List of Tables}

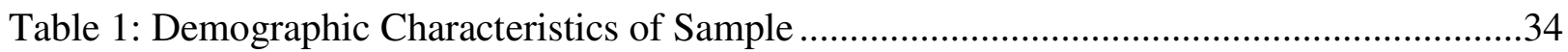

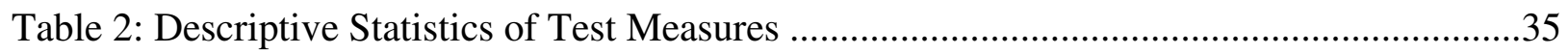

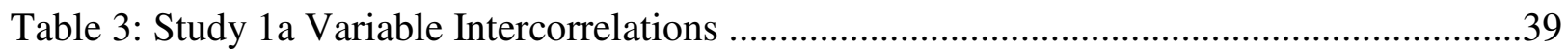

Table 4: Summary of Hierarchical Regression for Variables Predicting Changes in SIB Total by

Cognitive Fluctuation Severity ............................................................................. 41

Table 5: Study 1b Variable Intercorrelations ..............................................................45

Table 6: Study 2 Variable Intercorrelations .......................................................................47

Table 7: Summary of Hierarchical Regression for Variables Predicting Changes in ADFACS-

ADL Total by Cognitive Fluctuation Severity ............................................................48

Table 8: Summary of Hierarchical Regression for Variables Predicting Changes in ADFACS-

IADL Total by Cognitive Fluctuation Severity .............................................................51

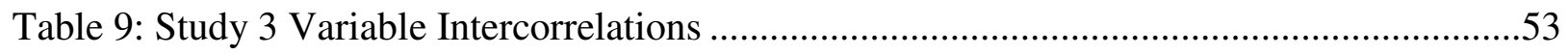

Table 10: Summary of Hierarchical Regression for Variables Predicting Changes in QUALID

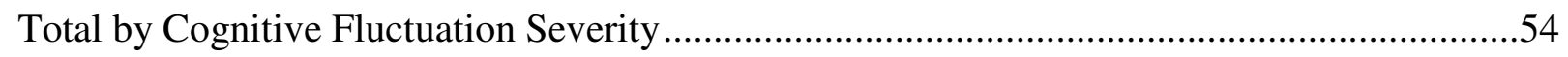

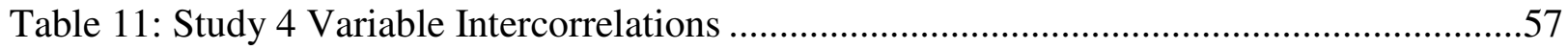

Table 12: Summary of Hierarchical Regression for Variables Predicting Changes in M-NCAS

Attitude Total by Cognitive Fluctuation Severity .......................................................60

Table 13: Summary of Hierarchical Regression for Variables Predicting Changes M-NCAS

Strain Total by Cognitive Fluctuation Severity .........................................................64

Table 14: Correlation of Variance Values and Scale Scores.............................................67

Table 15: Variable Intercorrelations for Medication Use and Charlson Comorbidity Index

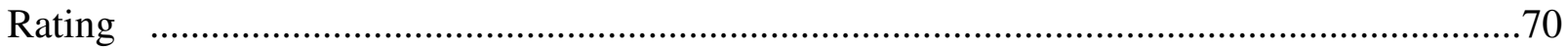


Table 16: Changes in Hierarchical Regression Models When 'SIB Total Score' is Added to Step

1 of Regression 


\section{Clinical and Functional Impact of Cognitive Fluctuations in Dementia}

According to the Alzheimer Society of Canada (2010) approximately 1 in 13 Canadians over the age of 65 years will develop Alzheimer's disease (AD) or a related dementia. Due to the rapidly increasing older adult population, the number of persons with dementia in Canada is projected to increase dramatically over the next several decades. Specifically, the proportion of the Canadian population aged 65 years and older has been steadily growing since 1960 , increasing from $8 \%$ at that time to $14 \%$ in 2009 . According to population projections, seniors are expected to comprise between 23-25\% of the population by 2036, and between 24-28\% by 2061 (Statistics Canada, 2010). By 2038, approximately 1,125,200 people will have dementia, which represents $2.8 \%$ of the Canadian population. This dramatic increase in dementia cases is projected to be associated with a cumulative economic burden of $\$ 872$ billion and a 10 -fold increase in the demand for long-term care (Alzheimer Society of Canada, 2010).

Cognitive fluctuations (CFs) are common among dementia patients, with an estimated frequency of occurrence of 80-90\% in dementia with Lewy bodies (DLB), $40 \%$ in vascular dementia (VaD) and 20\% in Alzheimer's disease (Lee, Taylor, \& Thomas, 2012). While the presence of CFs across dementia subtypes has been well established and has been characterized as debilitating, little is known about the characterization and clinical impact of these fluctuations in patients and their caregivers. The present study will investigate the nature of CFs in a sample of institutionalized patients with dementia, and will explore the extent to which these fluctuations are associated with patients' neuropsychological functioning, rate of cognitive decline, functional ability, and quality of life. This study will further examine the extent to which patients' CFs are associated with perceived distress among the institution's formal caregivers (i.e., nursing staff assigned to each patient). 


\section{Definition of Cognitive Fluctuations}

CFs are defined as spontaneous alterations in cognition, attention, and arousal (Escandon, Al-Hammadi, \& Galvin, 2010) that can range from transient black-outs to a delirious state and stupor (Franciotti et al., 2013). These episodes can occur with different frequencies, which vary from infrequent to several times per day (Franciotti et al., 2013). CFs are highly disabling, above and beyond the existing cognitive impairment, and can occur in most major subtypes of dementia; however, their prevalence is dependent on the dementia subtype, as noted above. Much of what is known about the clinical characteristics of CFs comes from DLB studies due to the particularly high prevalence of fluctuating cognition in this subgroup of patients with dementia. The Consensus Guidelines for the Clinical and Pathologic Diagnosis of Dementia with Lewy Bodies: Report of the Consortium on DLB International Workshop (McKeith et al., 2005) states that, in the earliest stages of DLB, patients may show CFs characterized by deficits in cognitive function and global performance that alternate with periods of normal (or nearnormal) performance. CFs often include pronounced variations in attention and alertness, as well as excessive daytime drowsiness. In contrast, some patients may experience improved performance in response to environmental novelty and increased arousal, but these positive affects are normally short lived (McKeith et al., 1996). Therefore, CFs can impact formal cognitive testing in some patients, making it difficult to provide a reliable assessment.

While common in dementia, the periodicity and amplitude of fluctuations are variable, both between subjects and within the same individual. They have been described as occurring rapidly (lasting minutes or hours), as well as more slowly (weekly or monthly). Therefore, substantial changes in mental status and behaviour can be observed both within the duration of a single interview and/or between consecutive examinations. Often, patients are able to identify the 
variable cognitive state themselves, but it is generally assumed that the most productive approach to identifying fluctuation is via a reliable informant. The differential diagnosis of CFs may include several conditions, including delirium due to medication toxicity or other concurrent illness. In addition, there are substantial difficulties inherent in defining and quantifying CFs, particularly later in the illness when CF variability may be hidden by progressive cognitive deterioration (McKeith et al., 1996).

CFs have been found to have a negative independent effect on activities of daily living in patients with dementia and are associated with an increased burden for caregivers (Lee et al., 2012). Although literary consensus supports the notion that CFs are common in patients with dementia, their accurate identification and assessment presents a major clinical challenge (Escandon et al., 2010; Lee et al., 2012). Recently, Lee et al. (2012) completed a comprehensive literature search to identify all the available literature on, and psychometric tests for, CFs in the major dementias of old age. Their search revealed a paucity of literature to guide clinicians in the accurate identification of CFs, which is surprising given that reviews and consensus criteria have long highlighted the importance of CFs in the diagnosis and differential diagnosis of dementia (Ballard et al., 2002; Escandon et al., 2010; Kolbeinsson \& Jónsson, 1993; McKeith et al., 2005; Robertsson, Blennow, Gottfries, \& Wallin, 1998). In fact, only three papers have reported on the development and validation of four psychometric instruments for identifying and rating CFs (Ferman, Smith, Boeve, \& Ivnik, 2004; Lee, McKeith, Mosimann, Ghosh-Nodial, et al., 2013; Walker, Ballard, et al., 2000). Three studies published prior to the development of these scales relied on experienced clinicians to identify CFs (Imamura et al., 1999; Walker et al., 1999; Walker, Ballard, et al., 2000) and nine studies used one or more of the three validated instruments for CFs to distinguish differences in the frequency of CFs between different groups 
of dementia patients (Ballard, O’Brien, Gray, \& Cormack, 2001; Bradshaw, Saling, Hopwood, Anderson, \& Brodtmann, 2004; Escandon et al., 2010; Ferman et al., 2004; Rongve, Brønnick, Ballard, \& Aarsland, 2010; Serrano \& García-Borreguero, 2004; Varanese, 2010; Walker, Ayre, et al., 2000; Walker, Ballard, et al., 2000).

\section{The Assessment of Cognitive Fluctuations}

Clearly, CFs are of significant importance due to their influence on patients' functioning and their diagnostic importance particularly in the early stages of dementia. As mentioned, very few measures are designed specifically to detect CFs in patients with dementia. This is partly due to mixed agreement regarding what constitutes fluctuations (Mega et al., 1996). However, several attempts have been made to develop reliable measures of CFs that can be used in clinical settings. Each new measure has built upon its predecessor in an attempt to increase the accuracy and user-friendliness of the scale. Each of these scales is described in detail below.

\section{Clinician Assessment of Fluctuation (CAF) and One Day Fluctuation Assessment}

(ODFAS). Walker, Ballard, et al. (2000) investigated the value of two scales developed to assess CFs in dementia by determining the agreement between a clinician's assessment of CFs and a scale completed by a non-clinician. In addition, they evaluated correlations of each of their scales with neuropsychological and electrophysiological markers of CFs. The CAF is the first of these two scales and is designed for use by experienced clinicians. The measure consists of a series of informant-directed screening questions regarding fluctuating confusion and impaired consciousness during the month prior to the assessment. Fluctuating confusion is rated 'present' if the informant provides a clear-cut example of an episode. If present, the frequency and duration of episodes of CFs are both rated on a scale of 0-4, and these scores are multiplied together to produce a severity score; a score of zero representing no CFs, 12 representing severe 
CFs, and a score of 16 representing a continuous clouded state, which, by definition, would denote no fluctuation but rather a stable state of confusion and impaired consciousness.

Their second scale, which is the non-clinician rated ODFAS, consists of seven items assessing confusional behaviours (i.e., falls, fluctuation, drowsiness, attention, disorganized thinking, altered level of consciousness, communication). Some items consist of multiple questions and scores are summed to produce a severity CF score ranging from 0 to 21 (Walker, Ballard, et al., 2000). The development of the ODFAS was based on a validated delirium assessment scale (Inouye et al., 1990), as well as the Barthel index (Mahoney, Wood, \& Wade, 1965) and the 'fluctuation' component of the DLB clinical diagnostic criteria (McKeith et al., 1996). The ODFAS asks informants to focus on the patient's level of fluctuating confusion over the day prior to the assessment and takes approximately 15 minutes to administer.

Since its initial publication, the CAF has been used in three other studies examining CFs (Bradshaw et al., 2004; Rongve et al., 2010; Varanese, 2010), but it has been reported to be difficult to use due to the descriptive and open-ended nature of several questions, and because it is heavily dependent on expert clinician skills to administer. As a result, the CAF is unlikely to gain popularity outside of its use in clinical research studies (Lee et al., 2012). The ODFAS appears to contain domains that overlap with CFs (e.g., attention, communication, level of consciousness), but others do not overlap at all (e.g., falls). In one study, the ODFAS was found to detect CFs in only $46 \%$ of patients with DLB (Bradshaw et al., 2004). Furthermore, high scores on the ODFAS may be influenced by fluctuations arising secondary to changes in medication regimens. Specifically, closer inspection of one DLB patient with an elevated score on the ODFAS in Bradshaw et al.'s (2004) study raised the possibility that the patient's fluctuations were actually related to the timing of levodopa administration in that patient. Finally, 
although both the ODFAS and CAF contain an open-ended question that allows for informantbased qualitative descriptions of CFs, which appear to differ between patients with AD and DLB, the quantitative scoring systems used does not take this type of information into account. Thus, the scoring methods used for these scales may miss important differences between patients with AD versus DLB (Bradshaw et al., 2004; Lee et al., 2012).

Mayo Composite Fluctuations Scale (MCFS). Ferman et al. (2004) examined whether certain aspects of fluctuations reliably distinguish DLB from AD and normal aging. They developed a 19-item questionnaire that is administered to informants and queries symptoms of fluctuations and delirium in the patient. The authors noted that, to some degree, most people can attest to experiencing or observing some variability in cognition, abilities, or alertness in themselves or others. Thus, for clinical purposes, identifying those aspects of fluctuations that are particularly salient for a particular dementia subtype and that can be consistently elicited from informant report could be very useful. To accomplish this task, Ferman et al. (2004) developed a brief questionnaire that asks the informant about the patient's behaviour during the past month. Initially, the questionnaire consisted of 19 items based on components of delirium and aspects of DLB fluctuations reported in previous studies (Inouye et al., 1990; McKeith et al., 1996; McKeith, Perry, Fairbairn, Jabeen, \& Perry, 1992). They found that the items that significantly differentiated AD from DLB included: 1) drowsiness and lethargy all the time or several times a day despite getting enough sleep the night before, 2) daytime sleep of 2 or more hours (before $7 \mathrm{pm}$ ), 3) staring into space for long periods, and 4) times when the patient's flow of ideas seem disorganized, unclear, and not logical. Thus, the authors incorporated only these four items into the final version of the MCFS. When using only these four items, normal older adults were unlikely to posses any of the four features of the fluctuations composite. One or two 
features were reported in over half of the patients with $\mathrm{AD}(58 \%)$ and in one-third (32\%) of the patients with DLB, indicating that both patient groups may have some of these characteristics. However, the presence of 3 or 4 features of the composite scale was found to be significantly more representative of the DLB patients (63\%) than AD patients (12\%) or normal elderly participants $(0.5 \%)$. Although rare, when features of the four-item scale were present in normal elderly participants, they were typically associated with very old age and lower global cognitive scores (Ferman et al., 2004).

In their initial study, Ferman et al. (2004) reported acceptable test-retest reliability for the four-item MCFS at a median interval of seven days for the DLB, AD, and normal elderly groups. This suggests that the informant's pattern of responding was consistent during a time frame short enough to assume a low likelihood of major changes occurring in the clinical feature of interest. However, the authors reported that clinician ratings of CFs were not correlated with the diagnoses of fluctuations based on the composite score. In response to this, the authors noted that the clinician's interview was unstandardized, and it was unknown exactly what questions were used, what aspects of fluctuations were specifically addressed, or whether there was unintended bias due to the clinician's knowledge of the patients' diagnostic group. This lack of congruence between standardized objective assessment of CFs and unstandardized clinicians' interviews further highlights the need for greater characterization of CFs that occur in various dementia subtypes. The authors noted that their findings point to a failure of their informant-based questionnaire format to tap into fluctuating cognitive abilities, despite it being fairly sensitive to other aspects of the phenomenon. The authors acknowledged that fluctuating cognition may be more amenable to an alternate measurement technique than that of fluctuating arousal and speech. However, other studies have suggested that patients with DLB perform significantly worse on 
tasks measuring attention compared with patients with $\mathrm{AD}$ of similar dementia severity (Ferman et al., 1999). Furthermore, DLB patients show greater variability within and between sessions on tasks of reaction time and vigilance compared to AD patients and normal controls (Ballard et al., 2001; Walker, Ayre, et al., 2000). Thus, it is possible that the MCFS is missing characteristics of fluctuations that can only be captured by more objective cognitive assessment, which can provide quantitative verification of task performance and variability with a degree of precision and objectivity that may not be possible with informant-based interviews or questionnaires.

The MCFS was further examined by Escandon et al. (2010), but the authors did not attempt to replicate the findings of the original paper and demonstrate its utility in identifying CFs in different dementia subgroups. Rather, they reported frequencies of positive responses to each of the four MCFS items in a mild AD group that were comparable to those in the AD group in Ferman et al. (2004), providing further support for the utility of these particular items in identifying CFs in patients with dementia.

The Dementia Cognitive Fluctuation Scale (DCFS). Lee et al. (2013) sought to develop a scale that is based on, but improves upon the CAF, ODFAS, and MCFS, in order to better facilitate the identification of CFs in dementia. MCFS items that sensitively distinguished between groups of patients with and without CFs were selected and included in the DCFS. The items included drowsiness, sleepiness, and staring into space. Items from the CAF and ODFAS that were considered to represent CFs (e.g., alertness, confusion, and daytime somnolence) were also included in the DCFS. Items that were not considered to relate to CFs (e.g., falls) were not included. The final version of the DCFS consists of 17-items under four domains: confusion, sleep, alertness and communication, which the authors applied cross-sectionally to patients with AD, VaD, DLB, dementia associated with Parkinson's disease (PDD), and normal controls. 
The authors found that four items from the original 17 -item version discriminated between patients with and without CFs. Their results suggested that CFs operate within three domains: 1) problems with consciousness (one item on the DCFS); 2) daytime sleepiness (two items on the DCFS); and 3) daytime functioning (one item on the DCFS). Furthermore, these particular test items are brief and can easily be incorporated into clinical practice.

Although this newly developed scale has not yet received widespread clinical or research application in the assessment of CFs, it does build on the most applicable items from previous scales and, thus, likely represents a superior measure for the presence of CFs. In the original report, Lee et al. (2013) found acceptable test-retest reliability for scale items, ranging from 0.51 to 0.84 , and inter-rater reliability, ranging from 0.52 to 0.95 . When the four test items are pooled to form a composite score, test-retest reliability is 0.71 and interrater reliability is 0.72 .

Early evidence suggests that the DCFS is a good measure for assessing the presence of CFs in patients with a range of dementia subtypes, but research examining the association between scores on the DCFS and other clinical factors related to care and quality of life, is currently lacking. However, previous research relying on other measures of CFs has revealed some important links between the presence of $\mathrm{CFs}$ and other central patient characteristics.

\section{Characteristics of Patients with CFs}

Cognitive functioning. The importance of CFs is evident from anecdotal reports identifying considerable changes in day to day functioning (Briel, McKeith, Barker, \& Hewitt, 1999; Hely, Reid, Halliday, \& McRitchie, 1996; Wagner \& Bachman, 1996). Previous work has shown a strong association between CFs and variability in attention and impairments in consciousness (Walker, Ayre, et al., 2000). However, many questions regarding the clinical impact of CFs remain unanswered. Because previous research on CFs has lacked objective 
methods of assessing their presence, it has been difficult to determine the degree of interference with cognitive performance that can be attributed to fluctuations. Most of what is known about the effect of fluctuations has been described in DLB (Escandon et al., 2010). Less is known about the extent to which CFs occur in other dementia subtypes and whether the presence of fluctuations impairs cognitive performance compared to patients with dementia who do not have features of fluctuations.

Ballard, Walker, O’Brien, and Rowan (2001) conducted a study comparing and characterizing the variability in cognitive performance in patients with DLB $(n=15)$ and AD $(n$ $=15)$, as well as healthy elderly controls $(n=10)$, over a 1-week period. Assessments were conducted in patients' homes using the Cognitive Drug Research Neuropsychological Test Battery, which is a well-validated computerized assessment (CDR; Simpson, Surmon, Wesnes, \& Wilcock, 1991). The version of the CDR used was specially designed for use in an elderly population and included measures of immediate verbal recognition, simple reaction time, choice reaction time, digit vigilance, spatial memory, memory scanning, and delayed verbal recognition (Simpson et al., 1991). Tasks were administered on three occasions across a 1-week period and CFs were assessed using the clinician administered CAF. They found that measures of variability (standard deviation and coefficient of variation) in measures of attentional performance (i.e., simple reaction time, choice reaction time, digit vigilance) across the 1 -week period were most strongly correlated with clinical CFs severity ratings. Variability in performance on measures of immediate and delayed verbal recognition, as well as numeric working memory, were also significantly correlated with CFs. Variability in spatial working memory was unrelated to severity of CFs. Ballard et al.'s (2001) results clearly demonstrate that variability in a number of 
cognitive domains can be detected using standardized neuropsychological assessment, confirming the broad impact of CFs on a range of cognitive tasks.

More recently, Escandon et al. (2010) examined the differences in neuropsychological performance in AD patients with and without CFs. Data were examined from 511 research participants aged 51 to 101 years who were enrolled in a longitudinal study of memory and aging. CFs were assessed using the MCFS (Ferman et al., 2004) and their neuropsychological battery assessed most of the major cognitive domains that are commonly compromised in $\mathrm{AD}$, including episodic memory, working memory, visuospatial skills, attention, and verbal fluency. They found an inverse correlation between the presence of CFs and composite score on the neuropsychological battery, as well as performance on the individual cognitive tests. Specifically, they found that the presence of CFs was associated with lower scores on measures of memory, visuospatial skills and working memory. The authors also examined partial correlations between each of the MCFS fluctuation variables and test performance and found that the item most strongly related to test performance was the 'illogical, disorganized thinking' variable. The weakest relationships were with the 'drowsy and lethargic' and 'stares into space' variables.

The pattern of cognitive deficits associated with CFs identified by Escandon et al. (2010) differs slightly from a similar study examining this association in a sample of patients with Parkinson's disease with dementia (Varanese, 2010). In this study, the authors found that the presence of $\mathrm{CFs}$, as assessed by the $\mathrm{CAF}$, was again associated with significantly reduced performance on a global measure of cognitive function (i.e., the Dementia Rating Scale Version 2). However, when performance on specific subscales of this measure was examined, they found that the presence of CFs was associated with impairment on measures of attention, memory, initiation, and perseveration. 
The results of these studies clearly demonstrate that the presence of CFs in patients with dementia worsen cognitive performance; however, the specific pattern of cognitive deficits remains unclear. Also, the use of the MCFS and CAF precluded the authors from an examination of the association between severity of CFs and degree of cognitive impairment because these scales do not include consideration of symptom severity. Instead, cut-off scores are used to classify participants into groups based on the presence or absence of CFs. Thus, additional research is needed to further characterize and assess the impact of CFs on patients' cognitive impairment.

Activities of daily living. Although limitations in cognitive capabilities are the most notable symptoms of degenerative dementias (Luttenberger, Schmiedeberg, \& Gräßel, 2012), patients' everyday practical capabilities are also important for independence (Robert et al., 2010; Sikkes, de Lange-de Klerk, Pijnenburg, \& Gillissen, 2012). These capabilities impact the timing of institutionalization and affect patient's quality of life, thereby influencing the degree of care that is required, and are thus significant contributors to the cost of health care (Jefferson, Paul, Ozonoff, \& Cohen, 2006). The ability to perform activities of daily living is considered a complex interaction of physical abilities, environmental conditions, and personal factors. Most studies examining everyday practical capabilities often differentiate between fundamental activities of daily living (ADLs; e.g. feeding one's self, bowel and bladder management, bathing, dressing, grooming, etc.) and instrumental ADLs (IADLs; e.g. housework, taking medications as prescribed, managing money, grocery shopping, etc.), the latter being more relevant in the context of mild dementia (Luttenberger et al., 2012; Robert et al., 2010; Sikkes et al., 2012).

Ballard, Walker, O’Brien, and Rowan (2001) completed the only study that examined the impact of CFs on ADLs. They examined an outpatient sample of 40 matched participants (15 
DLB, 15 AD, 10 elderly controls) who were assessed using the Activities of Daily Living Dependences (ADLD; Weintraub, Baratz, \& Mesulam, 1982). The authors found CFs, as assessed by the informant-based ODFAS (Walker, Ballard, et al., 2000), were strongly associated with total mean ADLD scores. Specifically, analysis of overall ADLD performance across all dementia groups revealed that CFs and dementia severity were important in influencing patients' ADLs; however, a diagnosis of AD or DLB did not significantly influence ADLD scores. Thus, irrespective of dementia subtype, CFs appear to represent an important predictor of lower ability to perform ADLs in patients with dementia.

Given the wide impact on a range of cognitive functions, it is not surprising that CFs are significantly associated with mean performance on ADLs. Although it could be argued that Ballard and colleagues' (2001) findings result from collinearity as it is possible that CFs and ADLs are both markers of dementia severity, this is unlikely as both CFs and Mini-mental State Examination (MMSE) score contributed independently to the level of impairment in activities of daily living (Ballard, Walker, et al., 2001). While this study was very informative, a more comprehensive assessment of specific symptoms of CFs and ADLs is required.

Quality of life. Recently, Mollenkopf and Walker (2007) described two broad categories of quality of life (QOL) indices. Objective indicators include standard of living and levels of chronic illness; the depth and breadth of interpersonal relationships with family and friends; and community resources, such as public transportation, good quality housing, and low crime rates. In contrast, subjective indicators are based in personal evaluations of life satisfaction, morale or happiness; perceived adequacy of food, financial resources, housing, and family relationships; and feeling valued or fully human. By virtue of the above-mentioned indicators, many patients with dementia would be considered to have poor QOL. Their objective resources are limited by 
their chronic illness, loss of personal relationships, and lack of connection to community resources. In addition, their losses can result in low morale and poor self-esteem (Keating \& Gaudet, 2012).

While QOL is an elusive concept that has been characterized and assessed in a variety of ways, health-related QOL is a somewhat narrower concept that relates to the impact of physical and mental disorders and disability on the general well being of a person. Boström, Jonsson, Minthon, and Londos (2007a) compared health-related QOL in patients with DLB and AD. A sample of 34 patients with DLB was matched to 34 patients with AD according to age, sex, and cognitive functioning. Participants were administered two widely used measures of QOL, one generic (the EQ-5D; The EuroQol Group, 1990) and one specifically developed to assess QOL in patients with dementia (the Quality of Life Scale - Alzheimer's disease; Logsdon, Gibbons, McCurry, \& Teri, 2002), either in their home or at a memory clinic together with their primary caregiver. They found that patients with DLB had significantly lower self-reported and informant-reported QOL compared to patients with AD. This finding is consistent with a number of previous studies assessing QOL in patients with dementia (Jönsson et al., 2006; Logsdon et al., 2002; Naglie et al., 2006; Sands, Ferreira, Stewart, Brod, \& Yaffe, 2004).

The results of Boström et al.'s (2007a) study, as well as another study of resource utilization in DLB versus AD (Boström et al., 2007b), indicate that the consequences of DLB and $\mathrm{AD}$ differ greatly in that a diagnosis of DLB predicts an almost 3-fold increase in resource utilization and a significantly lower QOL compared with AD. The authors identified several potential contributing factors that may explain this difference, including a greater presence of behavioural disturbances, reduced independence in IADLs and the presence of apathy and delusions. Another potential factor that may contribute to lower QOL in patients with DLB is the 
higher frequency of CFs in this dementia subtype. As mentioned, in the early stages of dementia CFs occur in approximately $20 \%$ of people with AD (Escandon et al., 2010; Lee et al., 2012) and upwards of $90 \%$ of people with DLB (Lee et al., 2012; McKeith et al., 2005). To date, no study has examined the association between severity of CFs and informant-based measures of QOL in patients with dementia.

\section{CFs and Burden of the Formal Caregiver.}

Providing care for institutionalized patients with dementia can be a daunting task for nursing staff (Beck, Ortigara, Mercer, \& Shue, 1999; Mahoney et al., 1999). Patients with dementia often exhibit disturbing behaviours, such as pacing, use of inappropriate behaviours, acting out, refusal of necessary care, hallucinations, and delusions (Herrmann et al., 2011; Kleinman, 2004). Thus, nursing staff caring for persons with dementia may have difficulty forming relationships with their patients, and the stress associated with care of such individuals has been proposed to contribute to high staff turnover (Kleinman et al., 2004). The turnover of regulated nursing staff and registered practical nurses in long-term care facilities is a pervasive problem in Canada (Chu, Wodchis, \& McGilton, 2013), but there is a scarcity of research examining this issue. The societal costs of nursing staff turnover are great, and can be expected to increase as the number of institutionalized older adults increases (Kleinman et al., 2004). Measuring caregiver burden in nurses caring for patients with dementia would allow for an investigation of specific factors, such as CFs, that could contribute to this burden. While such a

study has not yet been conducted in a long-term care setting, some work has been done focusing on informal caregivers (i.e., spouses and children of persons with dementia) in outpatient samples of patients with dementia. 
Lee et al. (2013) recently examined the specific impact of dementia subtype diagnosis and neuropsychiatric symptoms on the stress of a group of dementia caregivers. Study participants were community-dwelling caregivers (mainly spouses or children) of individuals with dementia recruited through dementia referrals from Old Age Psychiatry and Neurology National Health Services (NHS) in the North East of England. As part of their assessment, caregivers were administered the Carer Distress Scale (CDS) from The Neuropsychiatric Inventory (NPI; Cummings et al., 1994). The authors also administered the DCFS in order to examine the association between the presence of CFs in the individual with dementia and the presence and severity of caregiver distress. They found that caregivers of patients with dementia with a diagnosis of DLB or dementia associated with PDD reported significantly higher levels of caregiver stress than carers of patients with $\mathrm{AD}$ or $\mathrm{VaD}$. The high levels of caregiver stress were unrelated to age, gender, cognitive ability, activities of daily living, or motor functioning. Although psychosis and mood disturbances were most strongly associated with caregivers' distress, CFs also contributed significantly. As the authors note, the emergence of CFs is a common feature of dementia (McKeith et al., 2005) and the impact of these fluctuations is worthy of further investigation (Lee et al., 2013).

The significant impact of CFs on caregiver stress may be attributable to the unpredictability in the person's care needs from one moment to the next, as well as to the increased need to monitor the individual, increased difficulty in organizing and maintaining established care routines, and the need to remain flexible in terms of the level of support that is required (Lee et al., 2013). In Lee et al.'s (2013) study, many of the DCFS items assessing fluctuations did not correlate significantly with caregiver distress, as assessed by the CDS. However, the authors acknowledged that the use of the CDS as an assessment of caregiver 
distress may have limited their ability to adequately examine the contribution of factors other than neuropsychiatric symptoms (i.e., mood and psychotic symptoms) to caregiver distress because the use of the NPI necessarily focused caregivers' attention on mood and psychotic symptoms. Thus, a comprehensive assessment of caregiver distress is needed in order to more accurately identify the association between CFs and caregiver distress. Furthermore, while Lee et al. (2013) provide compelling evidence for an association between CFs and caregiver distress in a sample of individuals caring for dementia outpatients, the nature of this association remains untested in institutionalized care settings, where nurses act as the primary caregiver of long-term care patients.

\section{Present Study}

Given Canada's rapidly increasing older adult population and the projected dramatic increase in the number of patients with dementia over the next several decades, it is crucial to explore all factors that could potentially relate to patients' functional abilities and well-being. Gaining a better understanding of the clinical impact of CFs could lead to improved treatment planning for long-term care of patients. To this end, the purpose of the present study was to assess whether the presence and severity of CFs predicts a series of functional features in a sample of individuals with dementia living in a long-term care facility. To do this, the current study utilized the newly developed DCFS, which has been demonstrated to be a valid and reliable method for detecting the presence and severity of CFs. In addition, the clinical impact of CFs was explored by examining the association between CFs symptom severity and patients' current cognitive function, recent cognitive change, activities of daily living, and quality of life. This study also examined the impact of CFs on reports of caregiver burden from patients' primary nurses. The majority of previous studies examining this neurological phenomenon have 
been limited in that they did not assess the severity of CFs present in their samples. Thus, it has been difficult to determine the degree of interference with other clinical features, such as activities in daily living, which can be attributable to fluctuations. In addition, no previous study has examined the clinical impact of CFs on patients' primary nurses in an inpatient setting, where dementia severity is generally moderate-to-severe and patients require additional support.

Study aims and hypotheses. The current study has four primary aims. Given that literature examining the association between CFs and neuropsychological performance is sparse, and that those studies which have been published are troubled by their lack of symptom severity ratings (e.g., Ballard et al., 2001; Escandon et al., 2010; Varanese, 2010), the first goal of this study (Study Aim 1a) was to examine the association between CFs and cognitive test performance in a sample of institutionalized long-term care patients with dementia. It was expected that patients with more severe CFs ratings would exhibit greater deficits on cognitive measures. Specifically, it was hypothesized that: 1) patients who have more severe CFs would exhibit reduced performance on a measure of global cognitive function; and 2) subtest scores related to attention and memory skills would be most strongly associated with CFs symptom severity. In addition, retrospective longitudinal patient data collected from the Continuous Performance Scale (CPS) of the Minimum Data Set (MDS; Morris et al., 1994) was analyzed (Study Aim 1b) retrospectively to determine whether severity of CFs is associated with rate of recent cognitive decline. It was hypothesized that higher scores on the DCFS would be associated with a greater rate of decline on the CPS. Also, this study compared ratings on the DCFS to variability in objective measures of cognitive function. To do this, several measures known to be sensitive to CFs were administered to patients at three different time points within one week. These measures included the Trail Making Test A (TMT A; Reitan \& Wolfson, 1985), 
Digit Span Forward (DSF; Wechsler, 2008), and the Clock Drawing Test (CDT; Mendez, Ala, \& Underwood, 1992). The variability in each patient's performance across all three trials was compared to their score on the DCFS. It was hypothesized that higher scores on the DCFS would be associated with increased variability in cognitive test performance across the three trials.

The second aim of this study was to build on Ballard et al.'s (2001) work by taking a more comprehensive approach in exploring the influence of CFs on activities of daily living (Study Aim 2a) and instrumental ADLs (Study Aim 2b). To do this, patients' scores on the DCFS were compared to functional ratings recorded using an informant-rated measure of ADLs. This was the first study to examine the association between CFs and ADLs in an institutionalized setting. It was hypothesized that patients with more severe symptoms of CFs would exhibit greater impairment on measures of basic ADLs (e.g., bathing, feeding, etc.). Furthermore, it was hypothesized that greater severity of CFs would also predict greater impairment on measures of IADLs (e.g., preparing food, making phone calls, etc.).

The third aim of this study (Study Aim 3) was to explore whether CFs are a contributing factor in predicting patients' general QOL. Previous studies have found that DLB patients experience a lower QOL compared to patients with other forms of dementia, even when controlling for dementia severity (Boström, et al., 2007; Jönsson et al., 2006; Logsdon et al., 2002; Naglie et al., 2006; Sands et al., 2004). Given the finding that, in earlier disease stages, CFs occur much more frequently in DLB compared to other dementias (Escandon et al., 2010; Lee, McKeith, Mosimann, Ghosh-Nodial, et al., 2013; McKeith et al., 2005), it is possible that CFs may be accounting for some of the observed reduction of QOL in DLB sufferers. Extending this reasoning, it is possible that the presence of $\mathrm{CFs}$ is associated with lower QOL, regardless of dementia diagnosis. Therefore, it was hypothesized that greater severity of CFs would be 
negatively correlated with patients' QOL ratings. This was the first study to examine the relationship between CFs and quality of life in an institutionalized setting.

The fourth and final aim of this study was to investigate the extent to which the presence and severity of CFs is associated with caregivers' ratings of patients' attitude (Study Aim 4a) and the level of strain associated with their care (Study Aim 4b). Previous research has shown that caring for an individual with dementia can lead to significant levels of distress and negative health consequences for non-professional care providers (e.g., spouses, children; Buckner \& Yeandle, 2011; Hirst, 2005; Molyneux, McCarthy, McEniff, \& Cryan, 2008; Neil \& Bowie, 2008). Lee, McKeith, Mosimann, and Ghosh- Nodyal (2013) recently found that some symptoms of CFs (e.g., fluctuations in daytime somnolence), but not all, were associated with caregiver distress. However, their study focused on spouses, children, or friends of patients as the primary caregiver. The influence of CFs on self-reported caregiver burden has not been examined in institutionalized care settings. Thus, the current study assessed the relationship between CFs and caregiver burden by assessing distress levels in the primary nurse assigned to each patient. It was hypothesized that more severe CFs would predict a greater level of caregiver burden. 


\section{Method}

\section{Participants}

Although this study was exploratory in nature and many of the proposed comparisons had never been examined in an inpatient sample of patients with dementia, a previous study found medium to large effect sizes when examining the correlation between scores on the CAF and cognitive measures assessing verbal and visual memory, attention, reaction time, and numeric working memory in a sample of patients with AD or DLB (Ballard et al., 2001). Thus, for the main analysis, in order to similarly detect a medium effect size $\left(f^{2}=0.15\right)$ in this sample with power of .80 and an alpha of .05 for a hierarchical regression analysis with three control variables and one test variable, a prior power analysis using $\mathrm{G} *$ Power (Faul, Erdfelder, Buchner, \& Lang, 2009; Faul, Erdfelder, Lang, \& Buchner, 2007) determined that a sample size of 55 was needed. Therefore, this study included a sample size of 55 elderly residents, over the age of 65 years, who have been admitted to the veterans' long-term care facility at Sunnybrook Health Sciences Centre in Toronto, Ontario. The Sunnybrook Veterans Centre provides care for 500 veterans. Of the 500 beds, approximately half are dedicated for the care of veterans with moderate to severe dementia. The population is $94 \%$ male and the average age of residents is 90 years. Participants were recruited from the specialized cognitive support care units in the K-wing and L-wing only.

Each participant underwent a comprehensive diagnostic screening interview, as well as a review of his/her medical history to ensure that they met Diagnostic and Statistical Manual of Mental Disorders - Fourth Edition (DSM-IV; American Psychiatric Association, 2000) criteria for a primary degenerative dementia. This interview/review was conducted by Dr. Nathan Herrmann, $\mathrm{MD}, \operatorname{FRCP}(\mathrm{C})$, an experienced geriatric psychiatrist and associate scientist at the 
Brain Sciences Research Program at Sunnybrook Health Sciences Centre. Participants were automatically excluded if they lacked adequate eyesight or motor functioning to complete the test measures. A total of 88 participants were screened as potential participants. Of those, 55 were found to have adequate eyesight and motor functioning to participate. Demographic characteristics of this sample are discussed in the results.

\section{Measures}

Cognitive fluctuations. The presence of CFs was assessed using the Dementia Cognitive Fluctuations Scale (DCFS; Lee et al., 2013), discussed above. The DCFS is a recently developed scale that showed good levels of sensitivity, specificity, reliability, external validity, and internal consistency (Lee et al., 2013). The research version of the scale is based on four items from the original scale that were found to best discriminate between patients with and without CFs. The DCFS was administered to each patient's primary nurse, who responded to each item based on a five-point Likert scale. For example, the first item asks: "how great is the difference between the worst period of function and the best period of function on that day?” Potential responses include: "1. No difference (no impact on daily functioning);" "2. A slight difference (only a mild impact on daily functioning)," "3. A moderate difference (a clear impact on daily functioning),"

"4. A large difference (a severe impact on daily functioning)," and "5. A very large difference (a very severe impact on daily functioning." Nurses' ratings on each item were summed for a total score ranging from 4-20, with higher scores indicating more severe CFs. The internal consistency for the DCFS in the current study was poor $(\alpha=.52)$. Potential rationale and implications of this finding are discussed in the study's limitations.

To further validate the DCFS' ability to detect CFs, scores were compared to patients' variability on cognitive measures known to be sensitive to fluctuations that were administered on 
three occasions over a one-week period. These measures included the well-known Trail Making Test A (TMT A; Reitan \& Wolfson, 1985), Digit Span Forward (DSF; Wechsler, 2008), and the Clock Drawing Test (CDT; Mendez, Ala, \& Underwood, 1992). The TMT A requires participants to connect-the-dots on 25 consecutive targets (i.e., numbers) on a sheet of paper using a pencil. Performance on the TMT A can be scored in a number of ways. Typically, the amount of time taken to connect all the dots in order is used to determine participants' performance; however, this method has been judged to be less effective when administered to individuals with moderate-severe dementia because often time participants are unable to complete the entire task in the required time limit, if at all. For this reason, the current study examined participants' 'accuracy' on the test, which was based on the total number of correct lines completed. This method allows for partial points to be awarded to participants if they are able to connect at least two or more targets. Scores on this test can range from 0 to 24 . A similar modified scoring method has been used in previous research examining cognitive functioning in severe dementia (Binder, Storandt, \& Birge, 1999; Garbutt et al., 2008; Tartaglia et al., 2011).

The DSF task presents participants with a series of digits that they must immediately repeat back. If they do this successfully, they are given a longer digit list to recall. The total number of correctly repeated number sequences represents the total score on this task. The task is discontinued when participants respond incorrectly on two trials of the same digit length. Scores on this test can range from 0 to 16.

Finally, the CDT requires participants to draw a clock, put in all the numbers, and set the hands at ten past eleven. A wide variety of scoring systems have been published for this task (for a review, see Mainland \& Shulman, 2012). The current study used the scoring method described by Mendez et al. (1992), which rates participants' clock drawing on a series of 21 criteria. 
Sample scoring criteria include: "there is an attempt to indicate a clock in any way," "most symbols are distributed as a circle without major gaps," and "there are no repeated or duplicated number symbols." Scores on this measure can range from 0 to 21 .

Activities of daily living. Basic and IADLs were assessed using the Alzheimer's Disease Functional Assessment of Change Scale (ADFACS; Manero et al., 2014). The ADFACS is a functional assessment instrument widely used in clinical research. It is comprised of a 16-item scale measuring both basic ADLs and IADLs. Information can be collected from either the patient or the caregiver (or both). Each of the basic ADL items are scored based on a scale ranging from 0 (no impairment) to 4 (severe impairment) and each IADL item is scored on a scale ranging from 0 (no impairment) to 3 (severe impairment). The total score for the 16 -item scale ranges from 0 to 54, with higher scores indicating greater impairment. The present study found the internal consistency for the ADFACS scales to be excellent (ADL items, $\alpha=.92$; IADL items, $\alpha=.97)$

Quality of life. QOL was assessed using the 11-item Quality of Life in Late Stage Dementia scale (QUALID; Weiner et al., 2000), which was administered to each participant's primary nurse. The QUALID measures 11 observable behaviours indicating activity and emotional states. Ratings are made for observations made over the past seven days. Items are rated on a 5-point Likert scale (total score ranging from 11 to 55), with lower scores representing higher QOL. The measure takes approximately five minutes to administer. The QUALID has been demonstrated to be a reliable and valid scale for assessment of QOL (Weiner et al., 2000). The present study found the internal consistency for the QUALID scale to be excellent $(\alpha=.92)$.

Formal caregiver burden. Nursing care burden was assessed using the Modified Nursing Care Assessment Scale (M-NCAS; Kleinman et al., 2004). The M-NCAS is a 28-item 
nurse rating of burden associated with caring for institutionalized individuals with dementia. This measure assesses the extent to which certain behaviours and characteristics are present (attitude) and the extent to which these are a burden (strain; Herrmann et al., 2011). The MNCAS has been shown to be a valid and reliable means of obtaining care burden ratings from formal caregivers in long-term care settings (Kleinman et al., 2004). The present study found the internal consistency for the M-NCAS Attitude and Strain scales to be good to excellent $(\alpha=.84$ and $\alpha=.91$, respectively).

Cognitive deterioration over time. Longitudinal data assessing rate of cognitive decline over the past six months was analyzed retrospectively using previously collected scores on the Cognitive Performance Scale (CPS) from the Minimum Data Set (MDS; Morris et al., 1994). The CPS was initially developed to assess cognitive status of nursing home residents. It was developed using the Mini Mental Status Exam (MMSE; Folstein, Folstein, \& McHugh, 1975). The CPS assesses patient's awakening status (comatose or not) and patient's performance in four domains: short-term memory, ability to make decisions, ability to make self-understood, and ability to eat. Scores range from 0 to 6 , with high scores indicating worse cognition. The CPS is administered to patients at entry into the long-term care facility and at 6-month intervals following admission. Therefore, the two most recent CPS entries were examined for each patient as a method of investigating their level of recent cognitive decline.

Neuropsychological assessment. Participants completed the Severe Impairment Battery (SIB; Saxton, McGonigle-Gibson, Swihart, \& Miller, 1990). The SIB was developed to assess a range of cognitive abilities in patients who are too impaired to complete other standard neuropsychological assessment scales. Given that 30 minutes is considered the upper limit of the attention span of patients with severe dementia (Saxton et al., 1990), the SIB was designed to 
take approximately 20 minutes to administer. The test is designed to be well-structured and psychometrically reliable, while at the same time appearing to the patient as being more of an interview than a test in order to assist in maintaining the patient's attention for the duration of the 20-minute testing period. It is composed of simple one-step commands presented in conjunction with gestural cues and the measure allows for scoring credit for nonverbal and partially correct responses (Saxton et al., 1990). The SIB has been found to be a reliable and valid measure for objectively evaluating patients with dementia, particularly for those in the moderate to severe range of functioning (Panisset, Roudier, Saxton, \& Boiler, 1994; Schmidt, Wagner, \& Kiesler, 1999).

The SIB is divided into six subscales, including Attention, Orientation, Language, Memory, Visuoperception, and Construction. In addition, there are brief assessments of social skills, praxis, and responding to name. Examples of items from each subscale include: Attention - digit span, counting, auditory and visual stimuli; Orientation - name, date, city, time of day; Language - writing one's own name spontaneously, copying one's name, following simple verbal and written commands, naming shapes, naming photographs of objects, and naming real objects; Memory - immediate and delayed recall of previously presented objects, shapes, and colours, recalling a sentence and the examiner's name; Visuoperception - matching and identifying different colours and shapes; Construction - ability to draw shapes spontaneously, ability to copy and trace shapes. The total range of possible scores on the SIB is 0-152 (Saxton et al., 1990).

\section{Procedure}

This study was approved by the Research Ethics Boards at Sunnybrook Health Sciences Centre (REB \# 280-2013) and Ryerson University (REB \# 2013-278). Participants were 
recruited from the Dementia Veterans' Long-term Care facility at Sunnybrook Health Sciences Centre. They were identified as potential candidates for the study by Dr. Nathan Herrmann, MD, $\mathrm{FRCP}(\mathrm{C})$. If interested, patients were contacted by the primary researcher, who provided more information about the study. Informed consent was obtained from patients' legal representative by the primary investigator prior to the commencement of any study procedures.

Dr. Herrmann conducted an initial clinical interview and medical review with each patient in order to ensure that participants met DSM-IV criteria for a dementing disorder and to assign a specific dementia diagnosis (e.g., AD, VaD, DLB, etc.). The SIB took approximately 25 minutes to complete and was administered in the patient's unit. To compare the DCFS' ratings of CFs to variability in objective measures of fluctuations, three additional cognitive measures known to be sensitive to CFs (i.e., the TMT, DSF and CDT) were also administered during the first testing period, as well as on two additional days within one week following the initial appointment. These three tests took approximately 8 minutes to administer. The primary researcher and Dr. Gwen Li administered the cognitive measures. The primary researcher collected existing CPS data from participants' medical records in order to retrospectively assess rate of cognitive decline over the past six months.

Following the third testing session with each participant, the primary researcher met with the patient's primary nurse on call to complete the remaining study measures (i.e., ADFACS, QUALID, and M-NCAS). Completion of these questionnaires took approximately 20 minutes.

\section{Data Analysis}

The aforementioned measures yielded many variables of interest in the current analyses. CFs (as measured by the DCFS) were examined for their ability to predict: 1) cognitive performance (as measured by the SIB total score; Study Aim 1); 2) activities of daily living (as 
measured by the ADFACS-ADL [Study Aim 2a] score and the ADFACS-IADL score [Study Aim 2b]), 3) quality of life (as measured by the QUALID total score; Study Aim 3); and 4) caregiver burden (as measured by the M-NCAS Attitude score [Study Aim 4a] and the M-NCAS Strain score [Study Aim 4b]).

Missing data analysis. The extent and pattern of missing data are important factors for consideration when addressing missing data (Howell, 2007; Tabachnik \& Fidell, 2001). For example, the generalizability of results may suffer if the data are systematically missing (i.e., missing not at random [MNAR]). In contrast, data that is missing at random is seen as less problematic. For data to be considered missing completely at random (MCAR), it must satisfy the notion that the probability that an observation $\mathrm{X}_{\mathrm{i}}$ is missing is unrelated to the value of $\mathrm{X}_{\mathrm{i}}$ or to other variables in the analysis. If such a notion can be established, then various procedures can be undertaken to account for the missing data. Simple approaches, such as listwise deletion or pairwise deletion, are designed to deal with missing data, but these approaches have been criticized because the former results in a reduced sample size and the later results in the parameters of a model being based on different sets of data. Both situations lead to reduced statistical power (Howell, 2007). Mean substitution offers another approach for dealing with missing data, but this procedure adds no new information (the overall mean will be the same) and the process leads to an underestimation of error and biased estimates (Cohen, Cohen, West, \& Aiken, 2002).

Expectation maximization (EM) is an effective technique that is often used in data analysis to deal with missing data (for a detailed review of this technique, see Schafer, 1997; Schafer \& Olsen, 1998). EM overcomes many of the limitations of the above-mentioned techniques by using maximum likelihood equations that relate each variable to each other 
variable based on the means, variances, and covariances observed from the individuals whose data is completed. These formulas are then used to estimate the missing values (Cohen et al., 2002).

Given the importance of missing data for informing subsequent analysis decisions (Tabachnik \& Fidell, 2001), the dataset was examined for missing data points on all variables to be included in the analysis. The results confirmed that participants' number of years of education was the only variable of interest that contained missing data points (values missing for 17 of 55 participants). Participant's years of education were not recorded if the information was not contained within the patient's medical chart, if the patient's power-of-attorney was unable to provide the information, and if the patient was unable to provide a reliable description of their level of education. SPSS was used to conduct Little's (1988) MCAR test. This test was not statistically significant $\left(\chi^{2}[35]=17.45, p=.994\right)$, indicating that the data were likely missing completely at random and that estimating the missing values is unlikely to influence analysis results or their generalizability. Given this finding, missing data points were imputed using EM, which is a standard strategy for missing data imputation (Schlomer, Bauman, \& Card, 2010). This imputation and all subsequent analyses were conducted using SPSS v. 20.0.

Multiple Regression Analyses. The first aim of this study (Study Aim 1a) was to investigate the association between the presence and severity of CFs and cognitive test performance. As mentioned, cognitive test performance was evaluated using the SIB, which provides a global composite score, as well as subtest scores across various cognitive domains. A hierarchical multiple regression analysis was designed to predict SIB total scores. Predictors for this model included patients' age, education, and time since institutionalization, which are all variables that have previously been shown to influence cognitive test performance. Patients' 
ratings on the DCFS were also entered as a predictor. A theoretical entry order for potential predictor variables was predetermined as follows:

1. In the first block of this analysis, potential confounding variables that may be correlated with the outcome variable were entered. Specifically, research has demonstrated that differences in education (Ganguli et al., 1991), age (Ardila, Ostrosky-Solis, Rosselli, \& Gómez, 2000) and time since institutionalization (Wilson et al., 2007) may impact cognitive test scores. Entering each of these variables in Block 1 allowed for a less biased assessment of the incremental ability of CFs to predict cognitive test performance.

2. Next, to assess its incremental ability to predict cognitive test performance, patients' DCFS score was entered in Block 2.

The above model was constructed to systematically guide the regression analyses. After running the analyses, any variables that were not significantly correlated with the outcome variable were not included in the final regression model. Likewise, variables that did not incrementally predict SIB total score were removed before construction of the final model. Of note, many researchers run Pearson's correlations prior to regression analyses to determine which variables correlate with the outcome variable. This information is then used to preselect only those variables that correlate significantly with the outcome variable for entry into the regression model. However, this practice can result in missed relationships that are hidden by "suppressor effects." This occurs when a particular variable appears useless as a separate predictor, but may in fact change the prediction value of other variables and completely alter research outcomes (for a review on suppressor effects, see MacKinnon, Krull, \& Lockwood, 2000). For this reason, Pearson’s correlations were not used to preselect potential confounding variables in the current study. 
Following completion of the final model, assumptions of hierarchical regression were checked for potential violations.

This same method of model construction was used to determine if CFs predicted patients' ratings on measures assessing basic ADLs (as measured by ADFACS-ADL; Study Aim 2a), IADLs (as measured by ADFACS-IADL; Study Aim 2b), QOL (as measured by QUALID; Study Aim 3), and caregiver burden (as measured by M-NCAS Attitude and M-NCAS Strain; Study Aim 4a and Study Aim 4b). Potential confounding variables (i.e., age, education, and time since institutionalization) were entered into Block 1 of the model for each study only if prior research exists to suggest that they are associated with the outcome variable.

Bivariate Correlations. Bivariate correlations were examined to determine whether CFs were associated with variability in cognitive test performance over one week (Study Aim 1b). To determine patients' variability in cognitive test performance over time, coefficient of variation (CV) values were calculated for each test based on patients' scores on each of the three test days. The $\mathrm{CV}$ is a standardized measure of dispersion that is defined as the ratio of the standard deviation to the mean. The $\mathrm{CV}$ allows for a comparison of variability estimates (for a review of the use of CV to compare variability in performance, see Reed, Lynn, \& Meade, 2002) .

Bivariate correlations were also used to determine if CFs, as measured by the DCFS and by CV scores on the TMT, CDT, and DSF, are associated with scores on specific subtests of the SIB. Bivariate correlations were also used to determine whether CFs, as measured by patients' CV scores on the TMT, CDT, and DSF, are associated with ratings on measures of patients' ADLs, IADLs, QOL, and caregiver burden. In addition, retrospectively collected longitudinal data was examined to determine patients' degree of cognitive decline over six-month period, as measured by the difference in 
performance on the CPS at the patients' most recent administration compared to the administration completed six months prior. This difference was calculated by taking the patient's CPS score from their most recent administration (T2 score) and subtracting their CPS score from the administration conducted 6 months prior (T1 score). The resulting value was then divided by the T1 score to account for participants' baseline performance. Thus, the formula used for this calculation was as follows:

$$
(\mathrm{T} 2-\mathrm{T} 1) / \mathrm{T} 1
$$

Bivariate correlations were used to determine whether patients' CFs severity was associated with degree of change in CPS scores. 


\section{Results}

Demographic characteristics are provided in Table 1 . The majority of patients were male and Caucasian. The majority of the sample was born in Canada, with some participants born in Europe and one born in the Caribbean. Roughly half of the participants were married and onethird of the sample was widowed. The remainder was divorced, never married, or their marital status was unknown. The most common primary dementia diagnosis was $\mathrm{AD}$, followed by mixed dementia, $\mathrm{VaD}$, and PDD. After replacing missing data via EM, the median number of years of education was 12 , with a range of 3.02 to 26.91 years. The median age was 91 years, with a range of 81 to 97 years old. Finally, the median number of months since entering long-term care was 13.70 , with a range of less than one month to over 95 months.

\section{Descriptive Statistics}

Means, standard deviations, and range of scores for each variable of interest are provided in Table 2. In addition, standardized scores for skewness and kurtosis and results of significance testing for skewness and kurtosis are also displayed in Table 2. With the exception of some components of the SIB and DSF, all variables adequately approximated the normal distribution in the current sample. This information is provided to add to the description of each variable of interest. Importantly, hierarchical regression does not assume that the regressors have any distribution and, thus, non-normal variables do not need to be transformed. However, hierarchical regression does assume that the residuals in the model are random, normally distributed variables with a mean of zero (Cohen et al., 2002; Field, 2005). The degree to which this assumption was met in the current study will be discussed in detail in the Assumptions of Multiple Regression section below. 
Table 1

Demographic Characteristics of Sample

\begin{tabular}{|c|c|c|}
\hline \multirow[b]{2}{*}{ Characteristic } & \multicolumn{2}{|c|}{ Overall sample $(N=55)$} \\
\hline & $N$ & $\%$ \\
\hline Age $(M \pm \mathrm{SD})^{\mathrm{a}}$ & $90.41 \pm 2.84$ & \\
\hline Male & 49 & 89.10 \\
\hline \multicolumn{3}{|l|}{ Ethnicity } \\
\hline Caucasian & 52 & 94.55 \\
\hline Romanian & 1 & 1.82 \\
\hline Jamaican & 1 & 1.82 \\
\hline African-Canadian & 1 & 1.82 \\
\hline \multicolumn{3}{|l|}{ Birthplace } \\
\hline Canada & 48 & 87.27 \\
\hline Europe & 6 & 10.91 \\
\hline Caribbean & 1 & 1.82 \\
\hline Education in years actual $(M \pm \mathrm{SD})^{\mathrm{b}}$ & $12.30 \pm 3.00$ & \\
\hline Education in years $\mathrm{EM}(M \pm \mathrm{SD})^{\mathrm{c}}$ & $12.39 \pm 4.26$ & \\
\hline Months in long term care $(M \pm \mathrm{SD})^{\mathrm{d}}$ & $17.39 \pm 16.18$ & \\
\hline \multicolumn{3}{|l|}{ Marital Status } \\
\hline Married & 26 & 47.27 \\
\hline Widowed & 19 & 34.55 \\
\hline Divorced & 7 & 12.73 \\
\hline Never married & 1 & 1.82 \\
\hline Unknown & 2 & 3.64 \\
\hline \multicolumn{3}{|l|}{ Primary dementia diagnosis } \\
\hline Alzheimer's disease & 25 & 45.45 \\
\hline Vascular dementia & 8 & 14.55 \\
\hline Mixed dementia & 20 & 36.36 \\
\hline Parkinson's disease with dementia & 2 & 3.64 \\
\hline
\end{tabular}

Note: ${ }^{a}$ Range 81 to 97 years. Education information that was unavailable for 18 patients. ${ }^{b}$ Values represents sample prior to use of expectation maximization (EM) to estimate missing values $(n=$

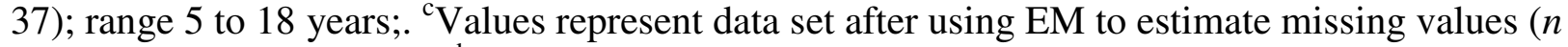
$=55$ ); range 3.02 to 26.91 . ${ }^{\mathrm{d}}$ Range 0.43 to 95.21 months. 
Table 2

Descriptive Statistics of Test Measures

\begin{tabular}{|c|c|c|c|c|}
\hline Measure & $\begin{array}{c}\text { Mean } \\
(S D)\end{array}$ & Range & Skewness & Kurtosis \\
\hline \multicolumn{5}{|l|}{ Cognitive function } \\
\hline SIB total score & $\begin{array}{c}86.65 \\
(13.77)\end{array}$ & $\begin{array}{l}15.00- \\
100.00\end{array}$ & $-3.09 * *$ & $13.21 * * *$ \\
\hline SIB social interaction & $\begin{array}{c}5.98 \\
(0.13)\end{array}$ & $\begin{array}{l}5.00- \\
6.00\end{array}$ & $-7.42 * * *$ & $55.00 * * *$ \\
\hline SIB memory & $\begin{array}{c}9.95 \\
(2.95)\end{array}$ & $\begin{array}{l}2.00- \\
14.00\end{array}$ & -0.64 & 0.32 \\
\hline SIB orientation & $\begin{array}{c}4.09 \\
(1.46)\end{array}$ & $\begin{array}{l}0.00- \\
6.00\end{array}$ & -0.35 & -0.31 \\
\hline SIB language & $\begin{array}{l}42.71 \\
(6.52)\end{array}$ & $\begin{array}{l}6.00- \\
48.00\end{array}$ & $-3.71 * * *$ & $18.45 * * *$ \\
\hline SIB attention & $\begin{array}{c}4.87 \\
(1.36)\end{array}$ & $\begin{array}{l}1.00- \\
6.00\end{array}$ & -1.27 & 1.13 \\
\hline SIB praxis & $\begin{array}{c}6.22 \\
(1.96)\end{array}$ & $\begin{array}{l}0.00- \\
8.00\end{array}$ & -1.02 & 0.76 \\
\hline SIB visuospatial & $\begin{array}{c}7.11 \\
(1.75)\end{array}$ & $\begin{array}{l}0.00- \\
8.00\end{array}$ & $-2.30 *$ & $5.33 * * *$ \\
\hline SIB construction & $\begin{array}{c}3.76 \\
(0.74)\end{array}$ & $\begin{array}{l}0.00- \\
4.00\end{array}$ & $-3.78 * * *$ & $15.03 * * *$ \\
\hline SIB orientation & $\begin{array}{c}1.96 \\
(0.27)\end{array}$ & $\begin{array}{l}0.00- \\
2.00\end{array}$ & $-7.42 * * *$ & $55.00 * * *$ \\
\hline TMT correct lines time 1 & $\begin{array}{l}17.33 \\
(9.12)\end{array}$ & $\begin{array}{l}0.00- \\
24.00\end{array}$ & -0.99 & -0.82 \\
\hline TMT correct lines time 2 & $\begin{array}{l}16.87 \\
(9.82)\end{array}$ & $\begin{array}{l}0.00- \\
24.00\end{array}$ & -0.96 & -0.92 \\
\hline TMT correct lines time 3 & $\begin{array}{c}16.52 \\
(10.39)\end{array}$ & $\begin{array}{l}0.00- \\
20.00\end{array}$ & -0.86 & -1.19 \\
\hline DSF total time 1 & $\begin{array}{l}7.47 \\
(2.64)\end{array}$ & $\begin{array}{l}0.00- \\
12.00\end{array}$ & -1.16 & 1.82 \\
\hline DSF time 2 & $\begin{array}{c}7.51 \\
(2.67)\end{array}$ & $\begin{array}{l}0.00- \\
15.00\end{array}$ & -0.37 & $2.12 *$ \\
\hline DSF time 3 & $\begin{array}{c}7.82 \\
(2.49)\end{array}$ & $\begin{array}{l}0.00- \\
15.00\end{array}$ & -0.68 & $3.12 * *$ \\
\hline
\end{tabular}


CDT total time 1

CDT total time 2

CDT total time 3
9.13

(6.13)

8.38

(6.66)

9.82

(6.75)

Quality of life

QUALID total score

18.25

(5.70)

Daily function

ADFACS-ADL

Nurse burden

M-NCAS Attitude total

51.22

Attention seeking

Autonomy

Difficulty

M-NCAS Strain total

Affect

Job satisfaction

Needy

Predictability

Self directed

Cognitive fluctuation

DCFS total score

18.02

(5.44)

14.73

(4.87)

18.47

(5.87)

46.35

(11.83)

9.91

(3.12)

7.53

(2.20)

11.29

(3.59)

9.02

(3.54)

8.60

(2.61)
$0.00-$

20.00

$0.00-$

20.00

$0.00-$

20.00
$-1.27$

$-1.28$

$-0.20$

$-1.32$

$11.00-$

35.00

1.23

1.33

$0.00-$

0.06

$-1.33$

30.00

$0.00-$

0.16

$-1.08$

23.00

$29.00-$ 79.00

10.00 -

30.00

$7.00-$

25.00

11.00 -

32.00

28.00 -

73.00

$6.00-$

18.00

$5.00-$

15.00

$7.00-$

19.00

$5.00-$

18.00

$5.00-$

15.00

0.40

$-0.71$

0.35

$-0.88$

0.22

$-0.88$

0.58

$-0.57$

0.08

$-0.80$

0.59

$-0.32$

0.95

1.36

0.58

$-0.67$

0.69

$-0.47$

0.26

$-0.70$

10.07

$5.00-$

0.25

$-0.88$ 
Note: SIB = Severe Impairment Battery (Saxton, McGonigle-Gibson, Swihart, \& Miller, 1990). TMT $=$ Trail Making Test (Reitan \& Wolfson, 1985). DSF $=$ Digit Span Forward (Wechsler, 2008). CDT $=$ Clock Drawing Test (Mendez, Ala, \& Underwood, 1992). QUALID = Quality of Life in Late Stage Dementia (Weiner et al., 2000). ADFACS = Alzheimer's Disease Functional Assessment and Change Scale; IADL = Instrumental Activities of Daily Living; ADL = Activities of Daily Living (Manero et al., 2014). M-NCAS = Modified Nursing Care Assessment Scale (Kleinman, Frank, Ciesla, Rupnow, \& Brodaty, 2004). DCFS = Dementia Cognitive Fluctuation Scale (Lee et al., 2013). ${ }^{*} p<.05 .{ }^{* *} p<.01 .{ }^{* * *} p<.00$ 


\section{Study Aim 1a: Predicting SIB Performance}

A series of hierarchical multiple regression models were conducted to determine whether CFs predicted level of cognitive functioning, as measured by the SIB.

\section{Model Construction}

Bivariate correlations between each potential predictor and SIB scores (in addition to all relevant variable intercorrelations) are reported in Table 3. The current hierarchical regression used SIB Total Score as the outcome variable, but correlations between each SIB subscale and each potential predictor are also reported in Table 3 for reference. Contrary to preliminary hypotheses, SIB Total Score was not significantly related to age $(r=.01, p$ $=.467)$, years of education $(r=-.04, p=.738)$, or number of months in long-term care $(r=.43$, $p=.087)$. SIB Total Score was, however, significantly correlated with cognitive fluctuations, as measured by the DCFS $(r=-.31, p=.010)$.

Table 4 displays the regression results for performance on the SIB. Age, years of education, and number of months in long-term care failed to account for unique variance in the outcome variable when entered into the hierarchical regression model at Step $1\left(R^{2}=0.03\right.$, $F(3,51)=0.05, p=.984)$. In Step 2, DCFS was added to the model and the variance in SIB Total scores accounted for by the model significantly increased, $\Delta R^{2}=0.10, F_{\text {change }}(1,50)=5.44, p$ $=.024$. In this model, DCFS emerged as the only significant predictor of SIB Total scores and the overall model again failed to reach significance, $R^{2}=0.10, F(4,50)=1.40, p=.247$. Because age, years of education, and number of months in long-term care did not significantly predict SIB Total scores when entered together in to the model they were eliminated as possible predictors and only DCFS was retained for the final regression model. 
Table 3

Study Aim 1a Variable Intercorrelations

\begin{tabular}{|c|c|c|c|c|c|c|c|}
\hline & 1 & 2 & 3 & 4 & 5 & 6 & 7 \\
\hline 1. Age & 1.00 & .04 & -.03 & -.09 & .01 & -.17 & -.08 \\
\hline 2. Education (years) ${ }^{\mathrm{a}}$ & & 1.00 & .19 & $.28 *$ & -.04 & .01 & -.03 \\
\hline 3. Months in long term care & & & 1.00 & .06 & .03 & .11 & .00 \\
\hline 4. DCFS Total & & & & 1.00 & $-.31 *$ & -.13 & -.21 \\
\hline 5. SIB Total Score & & & & & 1.00 & .12 & $.75 * * *$ \\
\hline 6. SIB Social Interaction & & & & & & 1.00 & $.28 *$ \\
\hline 7. SIB Memory & & & & & & & 1.00 \\
\hline 8. SIB Orientation & & & & & & & \\
\hline 9. SIB Language & & & & & & & \\
\hline 10. SIB Attention & & & & & & & \\
\hline 11. SIB Praxis & & & & & & & \\
\hline 12. SIB Visuospatial & & & & & & & \\
\hline 13. SIB Construction & & & & & & & \\
\hline 14. SIB Orientation to name & & & & & & & \\
\hline
\end{tabular}

Note: DCFS = Dementia Cognitive Fluctuation Scale (Lee et al., 2013). SIB = Severe Impairment Battery (Saxton, McGonigleGibson, Swihart, \& Miller, 1990). ${ }^{*} p<.05 .{ }^{* *} p<.01 .^{* * *} p<.001$. 
Table 3 (continued)

Study Aim la Variable Intercorrelations

\begin{tabular}{|c|c|c|c|c|c|c|c|}
\hline & 8 & 9 & 10 & 11 & 12 & 13 & 14 \\
\hline 1. Age & .24 & .02 & -.09 & .03 & -.07 & .13 & .07 \\
\hline 2. Education (years) & .11 & -.10 & .15 & -.15 & .02 & .01 & .00 \\
\hline 3. Months in long term care & .06 & -.04 & .05 & .14 & .10 & .03 & -.10 \\
\hline 4. DCFS Total & $-.43 * *$ & $-.28 *$ & -.08 & $-.27 *$ & -.21 & -.18 & -.22 \\
\hline 5. SIB Total Score & $.62 * * *$ & $.95 * * *$ & $.71 * * *$ & $.66 * * *$ & $.86 * * *$ & $.71 * * *$ & $.71 * * *$ \\
\hline 6. SIB Social Interaction & .10 & -.01 & -.01 & .16 & .09 & -.04 & -.02 \\
\hline 7. SIB Memory & $.39 * *$ & $.60 * * *$ & $.62 * * *$ & .24 & $.64 * * *$ & $.48 * * *$ & $.37 * *$ \\
\hline 8. SIB Orientation & 1.00 & $.52 * * *$ & $.34 *$ & $.43 * *$ & $.50 * * *$ & $.33^{*}$ & $.39 *$ \\
\hline 9. SIB Language & & 1.00 & $.57 * * *$ & $.58 * * *$ & $.77 * * *$ & $.72 * * *$ & $.77 * * *$ \\
\hline 10. SIB Attention & & & 1.00 & $.43^{* *}$ & $.58 * * *$ & $.46^{* * *}$ & $.39 * *$ \\
\hline 11. SIB Praxis & & & & 1.00 & $.56 * * *$ & $.38 * *$ & $.44 * *$ \\
\hline 12. SIB Visuospatial & & & & & 1.00 & $.52 * * *$ & $.56 * * *$ \\
\hline 13. SIB Construction & & & & & & 1.00 & $.69 * * *$ \\
\hline 14. SIB Orientation to name & & & & & & & 1.00 \\
\hline
\end{tabular}

Note: DCFS = Dementia Cognitive Fluctuation Scale (Lee et al., 2013). SIB = Severe Impairment Battery (Saxton, McGonigleGibson, Swihart, \& Miller, 1990). ${ }^{*} p<.05 .{ }^{* *} p<.01 .^{* * *} p<.001$. 
Table 4

Summary of Hierarchical Regression for Variables Predicting Changes in SIB Total by Cognitive Fluctuation Severity $(N=55)$

$B \quad S E$

\section{Step 1}

Constant

81.79

61.56

Education

$-1.59$

0.46

$-0.05$

Age at testing

0.07

0.68

0.01

Month in LTC

0.03

0.12

0.04

Step 2

Constant

Education

Age at testing

Months in LTC

DCFS Total
107.44

0.14

$-0.09$

0.03

$-1.48$
60.06

0.46

0.66

0.12

0.64
0.04

$-0.02$

0.04

$-0.33 *$

Note. $R^{2}=0.00$ for Step $1(p=.984), \Delta R^{2}=0.10$ for Step $2(p=.024)$. Model: $R^{2}=0.10$, $R_{\text {adj. }}^{2}=0.03 . * p<.05$. $* * p<.01 . * * * p<.001$. SIB $=$ Severe Impairment Battery (Saxton, McGonigle-Gibson, Swihart, \& Miller, 1990). LTC = Long term care. DCFS = Dementia Cognitive Fluctuation Scale. 


\section{Final Model}

A final simple linear regression model was conducted with DCFS as the predictor variable and SIB Total scores as the dependent variable. The overall model was significant, $R^{2}=$ $0.10, F(1,53)=5.69, p=.021$, and DCFS was found to significantly predict SIB Total scores, $\beta$ $=-0.31, t(54)=-2.39, p=.021$.

\section{Cross-Validation of Findings}

While the $R^{2}$ value presented above tells us how much of the variance in SIB Total scores is accounted for by the regression model in the current sample, the adjusted value estimates how much variance in SIB Total scores would be accounted for if the model had been derived from the population from which the sample was taken (derived via Wherry's equation for $R^{2}$; Field, 2005). This equation suggests that the final model predicting SIB Total scores would account for slightly less variance were the model built upon the population from which the sample was taken, $R_{\text {adj. }}^{2}=.08$. The model was further cross-validated using Stein's formula for a cross-validated $R^{2}$ (Field, 2005). This equation suggests that the final model of SIB Total scores would account for less variance were the model built upon population-wide data, $R_{\text {Stein adj. }}^{2}=0.05$.

\section{Assumptions of Regression}

In order to assess the validity and generalizability of these findings, variables included in the model were examined for violations of the assumptions of regression analysis. First, regression analysis assumes an absence of outliers and influential observations (Cohen, Cohen, West, \& Aiken, 2002; Field, 2005). In the above models, only one case had a value which represented an outlier (i.e., only one case had a standardized residual value > 3.27; Field, 2005). Similarly, only one case had extreme values for one or more of the predictor variables (i.e., had a leverage value $>[3[k+1\} / N]$; Field, 2005). However, review of the cases revealed that the data 
did not contain any influential observations (i.e., all Cook's $d$ values $<1.0$; Field, 2005). Thus, the case that represented an outlier on the predictor variables was retained in order to preserve the integrity of the regression parameters (Stevens, 2002).

In addition to an absence of outliers and influential observations, regression assumes an adequate sample size (Cohen et al., 2002; Field, 2005). A prior power analyses predicted that, given an anticipated medium effect size, 55 participants would be adequate (i.e., power $>0.80$; Field, 2005) for the four-predictor model outlined in the above model construction. To verify, a post hoc power analysis was conducted using G*Power (Faul et al., 2009, 2007) with the obtained sample size $(N=55)$, observed small-to-medium effect size $\left(R^{2}=0.101\right)$ and four predictors. The observed statistical power during the model construction was 0.68 . This indicates a slightly higher-than-anticipated possibility of Type II error in the first model and Type I error in the final model.

Further analyses explored possible violations of additional assumptions of regression. Intercorrelations between model predictors ranged from $r=-0.03$ to $r=0.28$. When considered together with collinearity diagnostic statistics (i.e., all tolerance values > .1 [range: .89 - .98]; all Variance Inflation Factor [VIF] values < 10 [range: 1.01 - 1.12], Field, 2005), the variables appear to demonstrate an acceptible absence of multicollinearity. Visual inspection of histograms revealed a slightly negatively skewed distribution of residuals; however, regression is quite robust to small violations of the normality assumption (McDonald, 2014).

Multiple regression also assumed homoscadasticity, which implies that the variance for residuals is equal across all values of the predictor variables, and that predictors are linearly related to the residuals (Field, 2005). Visual inspection of the bivariate scatter plots did not 
suggest any violations of these assumptions. Finally, the models also demonstrated indepedence of errors (Durbin-Watson statistics $=2.01$ ).

\section{Study Aim 1b: Cognitive Fluctuations and Variability in Cognitive Test Performance}

Bivariate correlations were examined to determine whether CFs are associated with variability in cognitive test performance over time. Two sets of cognitive test data were examined. The first set included coefficient of variation scores from the TMT, CDT, and DSF, which were administered to each patient on three separate occasions over a period of one week. Variables reflecting patients' age, total years of educations, and number of months in long-term care were also included in this analysis. Bivariate correlations between each variable of interest are presented in Table 5. The analysis revealed no significant associations between cognitive fluctuations, as measured by the DCFS, and coefficient of variation values for the TMT, CDT, or DSF. Higher years of education was found to be significantly associated with greater variability in TMT scores, $r=.28, p=.021$. In addition, greater coefficient of variation values for TMT scores was significantly associated with greater coefficient of variation values on the CDT, $r$ $=.571, p<.001$. Interestingly, coefficient of variation values for DSF scores was not significantly associated variation values for TMT or CDT scores.

The second set of cognitive test scores reflected changes in patients' performance on the CPS from the Minimum Data Set across the two most recent administrations of this measure. Bivariate correlations between each variable of interest and this change value are presented in Table 5. The analysis revealed no significant association between CFs, as measured by the DCFS, and change in CPS performance. In addition, change in CPS performance was not associated with CV values for the TMT or DSF, nor was it associated with patients' age, years of education, 
Table 5

Study Aim $1 b$ Variable Intercorrelations

\begin{tabular}{|c|c|c|c|c|c|c|c|c|}
\hline & 1 & 2 & 3 & 4 & 5 & 6 & 7 & 8 \\
\hline 1. Age & 1.00 & .04 & -.03 & -.09 & .00 & .05 & .10 & -.10 \\
\hline 2. Education (years) ${ }^{\mathrm{a}}$ & & 1.00 & .19 & $.28 *$ & -.09 & $-.31 *$ & .00 & .19 \\
\hline 3. Months in long term care & & & 1.00 & .06 & -.11 & -.04 & -.14 & -.22 \\
\hline 4. DCFS Total & & & & 1.00 & .09 & .09 & .08 & -.10 \\
\hline 5. CDT CV & & & & & 1.00 & $.57 * *$ & .17 & $.29 *$ \\
\hline 6. TMT CV & & & & & & 1.00 & .16 & .25 \\
\hline 7. DSF CV & & & & & & & 1.00 & .04 \\
\hline 8. MDS Change & & & & & & & & 1.00 \\
\hline
\end{tabular}

Note : DCFS = Dementia Cognitive Fluctuation Scale (Lee et al., 2013). TMT = Trail Making Test (Reitan \& Wolfson, 1985). DSF $=$ Digit Span Forward (Wechsler, 2008). CDT = Clock Drawing Test (Mendez, Ala, \& Underwood, 1992).. ${ }^{*} p<.05 .{ }^{* * *} p<.01 .{ }^{* * *} p$

$<.001$. MDS Change = Minimum Data Set Cognitive Performance Scale (Morris et al., 1994); change in score over past six months. 
or number of months in long-term care. Greater change in CPS performance was, however, significantly associated with higher CV values for the CDT, $r=.29, p=.035$.

\section{Study Aim 2a: Predicting ADFACS-ADL Score}

A hierarchical multiple regression model was constructed to determine whether CFs predicted patients' ability to engage in activities of daily living, as measured by the ADFACSADL.

\section{Model Construction}

Bivariate correlations between each potential predictor and ADFACS-ADL scores are presented in Table 6. Contrary to preliminary hypotheses, ADFACS-ADL score was not significantly related to age $(r=-.11, p=.222)$, years of education $(r=.08, p=.278)$, or number of months in long-term care $(r=-.18, p=.097)$. ADFACS-ADL scores were, however, significantly correlated with cognitive fluctuations, as measured by the DCFS $(r=.45, p<.001)$.

Table 7 displays the regression results for scores on the ADFACS-ADL. Age, years of education, and number of months in long-term care failed to account for unique variation in the outcome variable when entered into the hierarchical regression at Step $1\left(R^{2}=0.06, F(3,51)=\right.$ $1.06, p=.374)$. In Step 2, DCFS was added to the model and the variance in ADFACS-ADL scores accounted for by the model significantly increased, $\Delta R^{2}=0.19, F_{\text {change }}(1,50)=12.38, p$ $=.001$. Although DCFS emerged as the only significant predictor of ADFACS-ADL scores in

this model, the overall model was significant, $R^{2}=0.25, F(4,50)=4.07, p=.006$. Because age, years of education, and number of months in long-term care did not significantly predict ADFACS-ADL scores when entered together in to the model they were eliminated as possible predictors and only DCFS was retained for the final regression model. 
Table 6

Study Aim 2 Variable Intercorrelations

\begin{tabular}{|c|c|c|c|c|c|c|}
\hline & 1 & 2 & 3 & 4 & 5 & 6 \\
\hline 1. Age & 1.00 & .04 & -.03 & -.09 & -.11 & -.10 \\
\hline 2. Education (years) ${ }^{a}$ & & 1.00 & .19 & $.28^{*}$ & .08 & -.05 \\
\hline 3. Months in long term care & & & 1.00 & .06 & -1.78 & -.18 \\
\hline 4. DCFS Total & & & & 1.00 & $.45 * * *$ & .12 \\
\hline 5. ADFACS ADL & & & & & 1.00 & $.60 * * *$ \\
\hline 6. ADFACS IADL & & & & & & 1.00 \\
\hline
\end{tabular}

Note $:$ DCFS = Dementia Cognitive Fluctuation Scale (Lee et al., 2013). ADFACS = Alzheimer's Disease Functional Assessment and Change Scale; IADL = Instrumental Activities of Daily Living; ADL = Activities of Daily Living (Manero et al., 2014). ${ }^{*} p<.05 .{ }^{* *} p<.01 .{ }^{* * *} p<.001$. 
Table 7

Summary of Hierarchical Regression for Variables Predicting Changes in ADFACS-ADL

Total by Cognitive Fluctuation Severity $(N=55)$

\begin{tabular}{|c|c|c|c|}
\hline & $B$ & $S E$ & $\beta$ \\
\hline \multicolumn{4}{|l|}{ Step 1} \\
\hline Constant & 33.69 & 27.64 & \\
\hline Education & 0.19 & 0.21 & 0.12 \\
\hline Age at testing & -0.26 & 0.31 & -0.12 \\
\hline Month in LTC & -0.08 & 0.05 & -0.21 \\
\hline \multicolumn{4}{|l|}{ Step 2} \\
\hline Constant & 17.30 & 25.43 & \\
\hline Education & -0.01 & 0.20 & -0.01 \\
\hline Age at testing & -0.16 & 0.28 & -0.07 \\
\hline Months in LTC & -0.08 & 0.05 & -0.21 \\
\hline DCFS Total & 0.95 & 0.27 & $0.45 * *$ \\
\hline \multicolumn{4}{|c|}{$\begin{array}{l}\text { Note. } R^{2}=.06 \text { for Step } 1(p=.374), \Delta R^{2}=0.19 \text { for Step } 2(p=.001), \text { Model: } R^{2}=0.25, R_{\text {adj. }}^{2} \\
=0.19 . * p<.05 . * * p<.01 . * * * p<.001 . \text { LTC }=\text { Long term care. DCFS = Dementia Cognitive } \\
\text { Fluctuation Scale (Lee et al., 2013). ADFACS = Alzheimer's Disease Functional Assessment } \\
\text { and Change Scale; ADL = Activities of Daily Living (Manero et al., 2014). }\end{array}$} \\
\hline
\end{tabular}




\section{Final Model}

A final simple linear regression was conducted with DCFS as the only predictor variable and ADFACS-ADL scores as the outcome variable. The overall model was significant, $R^{2}=0.20$, $F(1,53)=13.12, p=.001$, and DCFS was found to significantly predict ADFACS-ADL scores, $\beta$ $=0.45, t(54)=3.62, p=.001$.

\section{Cross-Validation of Findings}

To supplement the $R^{2}$ value reported in the final model above, the adjusted $R^{2}$ was examined (derived from Wherry's equations). This equation suggests that the final model predicting ADFACS-ADL scores would account for slightly less variance were the model built upon the population from which the sample was taken, $R_{\text {adj. }}^{2}=.18$. The model was further crossvalidated using Stein's formula for a cross-validated $R^{2}$ (Field, 2005). This equation suggests that the final model of ADFACS-ADL scores would account for slightly less variance were the model built upon population-wide data, $R_{\text {Stein adj. }}^{2}=0.15$.

\section{Assumptions of Regression}

In testing adherence of these data to the assumptions of multiple regression, procedures and cut-off values applied were identical to those used in Study 1a. For the models in Study 2a, no variables had outliers. Additionally, while one case had extreme values for one or more of the predictor variables, this case (and all others) did not contain influential observations and was therefore not removed.

To determine the observed power for the three-predictor model outlined in the above model construction, a post hoc power analysis was run using $\mathrm{G}^{*}$ Power with the obtained sample size $(N=55)$, observed medium-to-large effect size $\left(R^{2}=0.25\right)$ and four predictors. The observed statistical power during model construction was 0.99 , indicating a low likelihood that 
the null hypothesis was incorrectly rejected in the detection of a significant effect for the model (i.e., risk of Type 1 error is low).

Intercorrelations between model predictors ranged from $r=-0.03$ to $r=0.28$. When considered together with collinearity diagnostic statistics (i.e., all tolerance values $>.1$ [range: .89 - .98]; all Variance Inflation Factor [VIF] values < 10 [range: $1.01-1.12$ ], Field, 2005), the variables appear to demonstrate an acceptible absence of multicollinearity. Visual inspection of histograms revealed that the residuals were adequately normally distributed. Bivariate scatterplots suggested adequate homoscedasticity. The assumption of independence of errors was not violated (Durbin-Watson statistic $=1.90$ ).

\section{Study Aim 2b: Predicting ADFACS-IADL Score}

A hierarchical multiple regression model was constructed to determine whether CFs predicted patients' ability to engage in activities of daily living, as measured by the ADFACSIADL.

\section{Model Construction}

Bivariate correlations between each potential predictor and ADFACS-IADL scores are presented in Table 6. Contrary to preliminary hypotheses, ADFACS-IADL score was not significantly related to age $(r=-0.10, p=.233)$, years of education $(r=-0.05, p=.351)$, number of months in long-term care $(r=-0.18, p=.092)$ or CFs, as measured by the DCFS $(r=0.12, p$ $=.184)$.

Table 8 displays the regression results for scores on the ADFACS-IADL. Age, years of education, and number of months in long-term care failed to account for unique variation in the outcome variable when entered into the hierarchical regression at Step $1\left(R^{2}=0.05, F(3,51)=\right.$ $0.79, p=.504)$. In Step 2, DCFS was added to the model and the variance in ADFACS-IADL 
Table 8

Summary of Hierarchical Regression for Variables Predicting Changes in ADFACS-IADL Total by Cognitive Fluctuation Severity $(N=55)$

$B \quad S E$

Step 1

Constant

51.09

41.74

Education

$-0.03$

0.31

0.11

Age at testing

$-0.36$

0.46

$-0.11$

Month in LTC

$-0.11$

0.08

$-0.18$

Step 2

Constant

Education

Age at testing

Months in LTC

DCFS Total
43.38

$-0.12$

$-0.31$

$-0.11$

0.45
42.47

0.33

0.46

0.08

0.45
$-0.06$

$-0.09$

$-0.18$

0.14

Note. $R^{2}=0.05$ for Step $1(p=.504), \Delta R^{2}=0.02$ for Step $2(p=.327)$. Model: $R^{2}=0.06, R_{\text {adj. }}^{2}$ $=-0.01 . * p<.05 . * * p<.01 . * * * p<.001$. LTC $=$ Long term care. DCFS $=$ Dementia Cognitive Fluctuation Scale. ADFACS-IADL $=$ ADFACS $=$ Alzheimer's Disease Functional Assessment and Change Scale; IADL = Instrumental Activities of Daily Living (Manero et al., 2014). 
scores accounted for by the model was not significantly increased, $\Delta R^{2}=0.02, F_{\text {change }}(1,50)=$ $0.98, p=.327$. The overall model was not significant, $R^{2}=0.06, F(4,50)=0.84, p=.507$.

Assumptions of regression were checked and, similar to Study 2a, no violations were found. As there were no variables that significantly predicted ADFACS-IADL, no additional analyses were necessary for Study $2 b$.

\section{Study Aim 3: Predicting QUALID Score}

A hierarchical multiple regression model was constructed to determine whether CFs predicted patients' quality of life, as measured by the QUALID.

\section{Model Construction}

Bivariate correlations between each potential predictor and QUALID scores are presented in Table 9. Contrary to preliminary hypotheses, QUALID total score was not significantly related to age $(r=-.08, p=.283)$ or number of months in long-term care $(r=-.19, p=.081)$. QUALID total score was, however, significantly correlated with CFs, as measured by the DCFS $(r=.29, p=.016)$.

Table 10 displays the regression results for scores on the QUALID. Age and number of months in long-term care failed to account for unique variation in the outcome variable when entered into the hierarchical regression at Step $1\left(R^{2}=0.04, F(2,52)=1.19, p=.311\right)$. In Step 2, DCFS was added to the model and the variance in QUALID scores accounted for by the model significantly increased, $\Delta R^{2}=0.09, F_{\text {change }}(1,51)=5.07, p=.029$. In this model, DCFS emerged

as the only significant predictor of QUALID scores and the overall model was not significant, $R^{2}$ $=0.13, F(3,51)=2.55, p=.066$. Because age and number of months in long-term care did not significantly predict QUALID scores when entered together into the model they were eliminated as possible predictors and only DCFS was retained for the final regression model. 
Table 9

Study Aim 3 Variable Intercorrelations

\begin{tabular}{lcccc}
\hline & 1 & 2 & 3 & 4 \\
\hline 1. Age & 1.00 & -.03 & -.09 & -.08 \\
2. Months in long term care & & 1.00 & .06 & -.19 \\
3. DCFS Total & & & 1.00 & $.29 *$ \\
4. QUALID & & & & 1.00
\end{tabular}

Note: DCFS = Dementia Cognitive Fluctuation Scale (Lee et al., 2013). QUALID = Quality of Life in Late Stage Dementia (Weiner et al., 2000). ${ }^{*} p<.05 .{ }^{* *} p<.01 .{ }^{* * *} p<.001$. 
Table 10

Summary of Hierarchical Regression for Variables Predicting Changes in QUALID Total by

Cognitive Fluctuation Severity $(N=55)$

$B \quad S E$

\section{Step 1}

Constant

34.99

24.67

Age at testing

$-0.17$

0.27

$-0.09$

Month in LTC

$-0.07$

0.05

$-0.19$

Step 2

Constant

Age at testing

Months in LTC

DCFS Total
24.83

$-0.12$

$-0.07$

0.55

24.18

0.26

$-0.06$

0.05

$-0.21$

0.25

Note. $R^{2}=.04$ for Step $1(p=.311), \Delta R^{2}=0.09$ for Step $2(p=.029)$, Model: $R^{2}=0.13, R_{\text {adj. }}^{2}$ $=0.08 . * p<.05 .{ }^{* *} p<.01 .{ }^{* * *} p<.001$. LTC $=$ Long term care. DCFS $=$ Dementia Cognitive Fluctuation Scale (Lee et al., 2013). QUALID = Quality of Life in Late Stage Dementia (Weiner et al., 2000). 


\section{Final Model}

A final simple linear regression was conducted with DCFS as the only predictor variable and QUALID total score as the outcome variable. The overall model was significant, $R^{2}=0.08$, $F(1,53)=4.82, p=.033$, and DCFS was found to significantly predict QUALID scores, $\beta=0.29$, $t(54)=2.19, p=.033$.

\section{Cross-Validation of Findings}

To supplement the $R^{2}$ value reported in the final model above, the adjusted $R^{2}$ was examined (derived from Wherry's equations). This equation suggests that the final model predicting QUALID scores would account for slightly less variance were the model built upon the population from which the sample was taken, $R_{\text {adj. }}^{2}=.29$. The model was further crossvalidated using Stein's formula for a cross-validated $R^{2}$ (Field, 2005). This equation suggests that the final model of QUALID total scores would account for much less variance were the model built upon population-wide data, $R_{\text {Stein adj. }}^{2}=0.03$.

\section{Assumptions of Regression}

In testing adherence of these data to the assumptions of multiple regression, procedures and cut-off values applied were identical to those used in Study 1a. For the models in Study 3, no variables had outliers. Additionally, while one case had extreme values for one or more of the predictor variables, this case (and all others) did not contain influential observations and were therefore not removed. In the above models, only one case had a value which represented an outlier on Y (i.e., only one case had a standardized residual value > 3.27; Field, 2005). However, no cases were identified as having extreme values for one or more of the predictor variables and no cases contained influential observations. Therefore, no cases were removed from the analyses. 
To determine the observed power for the three-predictor model outlined in the above model construction, a post hoc power analysis was run using G*Power with the obtained sample size $(N=55)$, observed small to medium effect size $\left(R^{2}=0.08\right)$ and three predictors. The observed statistical power during model construction was 0.59 . This indicates a slightly higherthan-anticipated possibility of Type II error in the first model and Type I error in the final model.

Intercorrelations between model predictors again ranged from $r=-0.09$ to $r=0.06$. When considered together with collinearity diagnostic statistics (i.e., all tolerance values $>.1$ [range: .98 - .99]; all Variance Inflation Factor [VIF] values $<10$ [range: $1.00-1.01$ ], Field, 2005), the variables appear to demonstrate an acceptible absence of multicollinearity. Visual inspection of histograms revealed that the residuals were adequately normally distributed. Bivariate scatterplots suggested adequate homoscedasticity. The assumption of independence of errors was not violated (Durbin-Watson statistic $=1.63$ ). Visual inspection of histograms revealed a slightly positively skewed distribution of residuals; however, as mentioned, regression is quite robust to small violations of the normality assumption (McDonald, 2014). Bivariate scatterplots likewise suggested adequate homoscedasticity.

\section{Study Aim 4a: Predicting M-NCAS Attitude Score}

A hierarchical multiple regression model was constructed to determine whether CFs predicted the presence of disruptive behaviours or characteristics, as measured by the M-NCAS Attitude score.

\section{Model Construction}

Bivariate correlations between each potential predictor and M-NCAS Attitude scores are presented in Table 11. Contrary to preliminary hypotheses, N-NCAS Attitude score was not significantly related to age $(r=-.17, p=.109)$. However, M-NCAS Attitude score was 
Table 11

Study Aim 4 Variable Intercorrelations

\begin{tabular}{|c|c|c|c|c|c|c|c|}
\hline & 1 & 2 & 3 & 4 & 5 & 6 & 7 \\
\hline 1. Age & 1.00 & -.03 & -.09 & -.17 & -.17 & -.04 & -.26 \\
\hline 2. Months in long term care & & 1.00 & .06 & -.23 & -.11 & $-.36 * *$ & -.06 \\
\hline 3. DCFS Total & & & 1.00 & $.31 *$ & $.33^{*}$ & .11 & $.41 * *$ \\
\hline 4. M-NCAS Attitude Domain Total & & & & 1.00 & $.72 * * *$ & $.75^{* * *}$ & $.69 * * *$ \\
\hline 5. M-NCAS Strain Domain Total & & & & & 1.00 & $.63 * * *$ & $.27 *$ \\
\hline \multicolumn{8}{|l|}{ M-NCAS Subscales } \\
\hline 6. Attention Seeking Subscale & & & & & & 1.00 & .21 \\
\hline 7. Autonomy Subscale & & & & & & & 1.00 \\
\hline 8. Difficulty Subscale & & & & & & & \\
\hline 9. Affect Subscale & & & & & & & \\
\hline 10. Job Satisfaction Subscale & & & & & & & \\
\hline 11. Needy Subscale & & & & & & & \\
\hline 12. Predictability Subscale & & & & & & & \\
\hline 13. Self-directed Subscale & & & & & & & \\
\hline
\end{tabular}


Table 11 (continued)

Study Aim 4 Variable Intercorrelations

\begin{tabular}{|c|c|c|c|c|c|c|}
\hline & 8 & 9 & 10 & 11 & 12 & 13 \\
\hline 1. Age & -.11 & -.24 & $-.30 *$ & .06 & .17 & -.09 \\
\hline 2. Months in long term care & -.10 & $-.32 * *$ & -.11 & -.11 & .12 & -.05 \\
\hline 3. DCFS Total & .22 & $.35^{* *}$ & $.30 *$ & .16 & $.32 *$ & .18 \\
\hline 4. M-NCAS Attitude Domain Total & $.85 * * *$ & $.68 * * *$ & $.50 * * *$ & $.55 * * *$ & $.54 * * *$ & $.55 * * *$ \\
\hline 5. M-NCAS Strain Domain Total & $.72 * * *$ & $.78 * * *$ & $.77 * * *$ & $.82 * * *$ & $.79 * * *$ & $.75 * * *$ \\
\hline 6. Attention Seeking & $.49 * * *$ & $.61 * * *$ & $.34 *$ & $.66 * * *$ & $.29 *$ & $.56 * * *$ \\
\hline 7. Autonomy Subscale & $.44 * *$ & $.37 * *$ & .19 & .05 & .23 & .24 \\
\hline 8. Difficulty Subscale & 1.00 & $.57 * * *$ & $.59 * * *$ & $.50 * * *$ & $.70 * * *$ & $.44 * *$ \\
\hline 9. Affect Subscale & & 1.00 & $.52 * * *$ & $.54 * * *$ & $.48 * * *$ & $.55 * * *$ \\
\hline 10. Job Satisfaction Subscale & & & 1.00 & $.55 * * *$ & $.61 * * *$ & $.44 * *$ \\
\hline 11. Needy Subscale & & & & 1.00 & $.51 * * *$ & $.53 * * *$ \\
\hline 12. Predictability Subscale & & & & & 1.00 & $.46^{* * *}$ \\
\hline 13. Self-directed Subscale & & & & & & 1.00 \\
\hline
\end{tabular}

Note: DCFS = Dementia Cognitive Fluctuation Scale (Lee et al., 2013). M-NCAS = Modified Nursing Care Assessment Scale (Kleinman, Frank, Ciesla, Rupnow, \& Brodaty, 2004). ${ }^{*} p<.05 .{ }^{* *} p<.01 .{ }^{* * *} p<.001$. 
significantly related to number of months in long-term care $(r=-.23, p=.045)$, as well as with cognitive fluctuations, as measures by the DCFS $(r=.31, p=.010)$. Education was not included as variable in the construction on this model because no previous research suggests that education level may be a relevant a prior covariate.

Table 12 displays the regression results for M-NCAS Attitude scores. Age and number of months in long-term care failed to account for unique variation in the outcome variable when entered into the hierarchical regression at Step $1\left(R^{2}=0.08, F(2,52)=2.40, p=.101\right)$. In Step 2, DCFS was added to the model and the variance in M-NCAS Attitude scores accounted for by the model significantly increased, $\Delta R^{2}=0.10, F_{\text {change }}(1,51)=6.05, p=.017$. In this model, DCFS emerged as the only significant predictor of M-NCAS Attitude scores; however, the number of months in long-term care variable approached significance as a predictor of M-NCAS scores $(\beta$ $=-0.24, \mathrm{t}(54)=-2.00, p=.051)$. The overall model was significant, $R^{2}=0.18, F(3,51)=3.77, p$ $=.017$. Because age did not significantly predict M-NCAS Attitude scores when entered into the model this variable was eliminated as a possible predictor. Patients' DCFS score and number of months in long-term care were retained for the final regression model.

\section{Final Model}

A final hierarchical regression was conducted with patients' number of months in longterm care entered in Step 1 and DCFS score entered in Step 2. Number of months in long-term care failed to account for unique variation in the outcome variable when entered in Step $1\left(R^{2}=\right.$ $0.05, F(1,53)=2.98, p=.090)$. In Step 2, DCFS was added to the model and the variance in MNCAS Attitude scores accounted for by the model significantly increased, $\Delta R^{2}=0.11$, $F_{\text {change }}(1,52)=6.57, p=.013$. The overall model was significant, $R^{2}=0.16, F(2,52)=4.93, p$ $=.011$ DCFS was found to significantly predict M-NCAS Attitude scores, $\beta=0.33, t(54)=2.66$, 
Table 12

Summary of Hierarchical Regression for Variables Predicting Changes in M-NCAS Attitude

Total by Cognitive Fluctuation Severity $(N=55)$

$B \quad S E$

Step 1

Constant

124.48

52.76

Age at testing

$-0.78$

0.58

$-0.18$

Month in LTC

$-0.18$

0.10

$-0.24$

Step 2

Constant

Age at testing

Months in LTC

DCFS Total
24.83

$-0.66$

$-0.20$

1.28

24.18

0.56

$-0.15$

$-0.25$

Note. $R^{2}=0.08$ for Step $1(p=.101), \Delta R^{2}=0.10$ for Step $2(p=.017)$, Model: $R^{2}=0.18, R^{2}$ adj. $=0.13$. $* p<.05$. $* * p<.01$. $* * * p<.001$. LTC $=$ Long term care. DCFS = Dementia Cognitive Fluctuation Scale (Lee et al., 2013). M-NCAS = Modified Nursing Care Assessment Scale (Kleinman, Frank, Ciesla, Rupnow, \& Brodaty, 2004). 
$p=.013$. The number of months in long-term care variable again approached significance as a predictor of M-NCAS Attitude scores $(\beta=-0.25, t(54)=-1.96, p=.055)$. Because patients' number of months in long-term care did not significantly account for unique variation in the outcome variable, a simple linear regression was conducted with DCFS scores as the only predictor. The effect size (i.e., $R^{2}$ ) of the model produced in this analysis was 0.10 , which is slightly less than the effect size of the model that retained number of months in long-term care as a variable $\left(R^{2}=0.16\right)$. Thus, patients' number of months in long-term care was retained as a predictor variable in the final model.

\section{Cross-Validation of Findings}

To supplement the $R^{2}$ value reported in the final model above, the adjusted $R^{2}$ was examined (derived from Wherry's equations). This equation suggests that the final model predicting M-NCAS Attitude scores would account for slightly less variance were the model built upon the population from which the sample was taken, $R_{\text {adj. }}^{2}=0.13$. The model was further cross-validated using Stein's formula for a cross-validated $R^{2}$ (Field, 2005). This equation suggests that the final model of M-NCAS Attitude scores would account for slightly less variance were the model built upon population-wide data, $R_{\text {Stein adj. }}^{2}=0.11$.

\section{Assumptions of Regression}

In testing adherence of these data to the assumptions of multiple regression, procedures and cut-off values applied were identical to those used in Study 1a. For the models in Study 4a, no variables had outliers on Y. Additionally, while one case had extreme values for one or more of the predictor variables, this case (and all others) did not contain influential observations and was therefore not removed. 
To determine the observed power for the three-predictor model outlined in the above model construction, a post hoc power analysis was run using G*Power with the obtained sample size $(N=55)$, observed medium-to-large effect size $\left(R^{2}=0.18\right)$ and three predictors. The observed statistical power during model construction was 0.93 , indicating a low likelihood that the null hypothesis was incorrectly rejected in the detection of a significant effect for the model (i.e., risk of Type 1 error is low).

Intercorrelations between model predictors ranged from $r=-0.09$ to $r=0.06$. When considered together with collinearity diagnostic statistics (i.e., all tolerance values $>.1$ [range: .98 - .99]; all Variance Inflation Factor [VIF] values < 10 [range: 1.00 - 1.01], Field, 2005), the variables appear to demonstrate an acceptible absence of multicollinearity. Visual inspection of histograms revealed that the residuals were adequately normally distributed. Bivariate scatterplots likewise suggested adequate homoscedasticity. The assumption of independence of errors was not violated (Durbin-Watson statistic $=2.00$ ). Visual inspection of histograms revealed that the residuals were adequately normally distributed. Bivariate scatterplots suggested adequate homoscedasticity.

\section{Study Aim 4b: Predicting M-NCAS Strain Score}

A hierarchical multiple regression model was constructed to determine whether CFs predicted nurse strain, as measured by the M-NCAS Strain score.

\section{Model Construction}

Contrary to preliminary hypotheses, M-NCAS Strain score was not significantly related to age $(r=-.17, p=.106)$ or number of months in long-term care $(r=-.11, p=.207)$. M-NCAS Strain score was, however, significantly correlated with CFs, as measured by the DCFS ( $r=.33$, $p=.007)$. 
Table 13 displays the regression results for scores on the M-NACS Strain domain. Age and number of months in long-term care failed to account for unique variation in the outcome variable when entered into the hierarchical regression at Step $1\left(R^{2}=0.04, F(2,52)=1.17, p\right.$ $=.319)$. In Step 2, DCFS was added to the model and the variance in M-NCAS Strain scores

accounted for by the model significantly increased, $\Delta R^{2}=0.11, F_{\text {change }}(1,51)=6.33, p=.015$. In this model, DCFS emerged as the only significant predictor of M-NCAS Strain scores and the overall model was significant, $R^{2}=0.15, F(3,51)=2.97, p=.040$. Because age and number of months in long-term care did not significantly predict M-NCAS Strain scores when entered together into the model they were eliminated as possible predictors and only DCFS was retained for the final regression model.

\section{Final Model}

A final simple linear regression was conducted with DCFS as the only predictor variable and M-NCAS Strain total score as the dependent variable. The overall model was significant, $R^{2}$ $=0.11, F(1,53)=6.56, p=.013$, and DCFS was found to significantly predict M-NCAS Strain scores, $\beta=0.33, t(54)=2.56, p=.013$.

\section{Cross-Validation of Findings}

To supplement the $R^{2}$ value reported in the final model above, the adjusted $R^{2}$ was examined (derived from Wherry's equations). This equation suggests that the final model predicting M-NCAS Strain scores would account for slightly less variance were the model built upon the population from which the sample was taken, $R_{\text {adj. }}^{2}=0.09$. The model was further cross-validated using Stein's formula for a cross-validated $R^{2}$ (Field, 2005). This equation suggests that the final M-NCAS Strain score model would account for less variance were the model built upon population-wide data, $R_{\text {Stein adj. }}^{2}=0.06$. 
Table 13

Summary of Hierarchical Regression for Variables Predicting Changes in M-NCAS Strain

Total by Cognitive Fluctuation Severity $(N=55)$

$B \quad S E$

Step 1

Constant

113.59

51.24

Age at testing

$-0.73$

0.57

$-0.18$

Month in LTC

$-0.09$

0.10

$-012$

Step 2

Constant

Age at testing

Months in LTC

DCFS Total
90.26

$-0.61$

$-0.10$

1.27

49.68

0.54

$-0.15$

$-0.14$

0.10

$0.32 *$

Note. $R^{2}=0.04$ for Step $1(p=.319), \Delta R^{2}=0.11$ for Step $2(p=.0154)$, Model: $R^{2}=0.15$. $* p<.05$. $* * p<.01$. $* * * p<.001$. LTC $=$ Long term care. DCFS = Dementia Cognitive Fluctuation Scale (Lee et al., 2013). M-NCAS = Modified Nursing Care Assessment Scale (Kleinman, Frank, Ciesla, Rupnow, \& Brodaty, 2004). 


\section{Assumptions of Regression}

In testing adherence of these data to the assumptions of multiple regression, procedures and cut-off values applied were identical to those used in Study 1a. For the models in Study 4b, no variables had outliers. Additionally, while one case had extreme values for one or more of the predictor variables, this case (and all others) did not contain influential observations and was therefore not removed.

To determine the observed power for the three-predictor model outlined in the above model construction, a post hoc power analysis was run using $\mathrm{G}^{*}$ Power using the obtained sample size $(N=55)$, observed medium to large effect size $\left(R^{2}=0.15\right)$ and three predictors. The observed statistical power during model construction was 0.85 , indicating a low likelihood that the null hypothesis was incorrectly rejected in the detection of a significant effect for the model (i.e., risk of Type 1 error is low).

Intercorrelations between model predictors ranged from $r=-0.09$ to $r=0.06$. When considered together with collinearity diagnostic statistics (i.e., all tolerance values $>.1$ [range: .98 - .99]; all Variance Inflation Factor [VIF] values < 10 [range: 1.00 - 1.01], Field, 2005), the variables appear to demonstrate an acceptible absence of multicollinearity. Visual inspection of histograms revealed that the residuals were adequately normally distributed. Bivariate scatterplots suggested adequate homoscedasticity. The assumption of independence of errors was not violated (Durbin-Watson statistic $=2.01$ ).

\section{Association Between M-NCAS Subtest Scores and Cognitive Fluctuations}

Bivariate correlations were examined to determine whether CFs are associated with specific M-NCAS subscales. Patients' age, total years of education, and number of months in long-term care were also included in this analysis. Bivariate correlations between each variable 
of interest are presented in Table 11. The analysis revealed that greater severity of CFs, as measured by the DCFS, is associated with increased scores on the Autonomy $(r=.41, p=.002)$, Affect $(r=.35, p=.008)$, Job Satisfaction $(r=.30, p=.027)$, and Predictability $(r=.32, p$ $=.019)$ M-NCAS subscales. In addition, increased patient age is associated with a decrease in strain score on the Job Satisfaction subscale $((r=-.30, p=.027)$ and greater months spent in long-term care is associated with a decrease in strain score on the Attention Seeking $(r=-.36, p$ $=.006)$ and Affect $(r=-.32, p=.018)$ subscales.

\section{Association Between Cognitive Test Variability and Other Study Measures}

Bivariate correlations were examined to determine whether variability in TMT, CDT, and DSF scores across three testing sessions is associated with level of cognitive function as assessed by the various SIB subscales. Results of these analyses are presented in Table 14. Higher CV values for the DSF was significantly associated with lower performance on the Memory $(r=-.30$, $p=.026)$, Attention $(r=-.41, p=.002)$, and Visuospatial $(r=-.39, p=.003) \mathrm{SIB}$ subscales. Also, higher CV values for the CDT was significantly associated with lower performance on the Orientation $(r=-.31, p=.022)$, Attention $(r=-.28, p=.038)$, and Praxis $(r=-.27, p=.046)$ subscales. Finally, higher CV values for the TMT was significantly associated with lower performance on the Orientation $(r=-.27, p=.049)$, Attention $(r=-.41, p=.002)$, and Praxis $(r=$ $-.28, p=.042)$ subscales.

Bivariate correlations were also examined to determine whether variability in TMT, CDT, and DSF scores across three testing sessions is associated with scores on the M-NCAS, ADFACS, and QUALID. Results of these analyses are also presented in Table 14. Higher CV values for the CDT was significantly associated with greater impairment in IADLs $(r=.36, p$ $=.006)$, as well as ADLs $(r=.28, p=.041)$, as assessed by the ADFACS. Also, higher CV 


\begin{tabular}{|c|c|c|c|c|c|c|c|c|c|}
\hline & 1 & 2 & 3 & 4 & 5 & 6 & 7 & 8 & 9 \\
\hline 1. CDT Variability & 1.00 & $.57 * * *$ & .17 & .10 & .08 & $.36^{* *}$ & $.27 *$ & .03 & .04 \\
\hline 2. TMT Variability & & 1.00 & .16 & -.03 & -.10 & $.28 *$ & $.29 *$ & .09 & -.02 \\
\hline 3. DSF Variability & & & 1.00 & -.09 & -.18 & -.17 & .18 & -.12 & .02 \\
\hline 4. M-NCAS Attitude Domain & & & & 1.00 & $.72 * * *$ & $.43^{* *}$ & $.38 * *$ & $.55 * * *$ & $-.27 *$ \\
\hline 5. M-NCAS Strain Domain & & & & & 1.00 & .12 & .14 & $.43 * *$ & $-.31 *$ \\
\hline 6. ADFACS IADL & & & & & & 1.00 & $.60 * * *$ & $.29 *$ & -.19 \\
\hline 7. ADFACS ADL & & & & & & & 1.00 & .26 & -.13 \\
\hline 8. QUALID & & & & & & & & 1.00 & .05 \\
\hline SIB Subscale Scores & & & & & & & & & 1.00 \\
\hline 9. Social Interaction & & & & & & & & & \\
\hline 10. Memory & & & & & & & & & \\
\hline 11. Orientation & & & & & & & & & \\
\hline 12. Language & & & & & & & & & \\
\hline 13. Attention & & & & & & & & & \\
\hline 14. Praxis & & & & & & & & & \\
\hline 15. Visuospatial & & & & & & & & & \\
\hline 16. Construction & & & & & & & & & \\
\hline 17. Orientation to Name & & & & & & & & & \\
\hline
\end{tabular}

Note: TMT $=$ Trail Making Test (Reitan \& Wolfson, 1985). DSF = Digit Span Forward (Wechsler, 2008). CDT = Clock Drawing Test (Mendez, Ala, \& Underwood, 1992). SIB = Severe Impairment Battery (Saxton, McGonigle-Gibson, Swihart, \& Miller, 1990). QUALID = Quality of Life in Late Stage Dementia (Weiner et al., 2000). ADFACS = Alzheimer's Disease Functional Assessment and Change Scale; IADL = Instrumental Activities of Daily Living; ADL = Activities of Daily Living (Manero et al., 2014). M-NCAS $=$ Modified Nursing Care Assessment Scale (Kleinman, Frank, Ciesla, Rupnow, \& Brodaty, 2004). ${ }^{*} p<.05$. $^{* *} p<.01{ }^{* * * *} p<.001$. 
Table 14 (continued)

Correlation of Variance Values and Scale Scores

\begin{tabular}{|c|c|c|c|c|c|c|c|c|}
\hline & 10 & 11 & 12 & 13 & 14 & 15 & 16 & 17 \\
\hline 1. CDT Variability & -.19 & $-.31 *$ & -.16 & $-.28 *$ & $-.27 *$ & -.12 & -.10 & .13 \\
\hline 2. TMT Variability & -.15 & $-.27 *$ & -.14 & $-.41 * *$ & $-.28 *$ & -.19 & .04 & .01 \\
\hline 3. DSF Variability & $-.30 *$ & -.13 & -.17 & $-.41 * *$ & -.15 & $-.39 * *$ & -.15 & .10 \\
\hline 4. M-NCAS Attitude Domain & -.13 & -.25 & -.01 & -.01 & $-.36 * *$ & -.09 & -.04 & -.03 \\
\hline 5. M-NCAS Strain Domain & -.12 & -.19 & .04 & .14 & $-.29 *$ & .01 & -.03 & -.02 \\
\hline 6. ADFACS IADL & $-.34 *$ & $-.41 * *$ & $-.28 *$ & -.25 & -.24 & -.18 & -.18 & -.19 \\
\hline 7. ADFACS ADL & $-.43 * *$ & $-.44 * *$ & $-.50 * * *$ & $-.39 * *$ & $-.49 * * *$ & $-.42 * *$ & -.23 & -.26 \\
\hline 8. QUALID & .07 & -.25 & .04 & .02 & -.21 & -.02 & .03 & .03 \\
\hline \multicolumn{9}{|l|}{ SIB Subscale Scores } \\
\hline 9. Social Interaction & $.28 *$ & .10 & -.01 & -.01 & .16 & .09 & -.04 & -.02 \\
\hline 10. Memory & 1.00 & $.39 * *$ & $.60 * * *$ & $.62 * * *$ & .24 & $.63 * * *$ & $.48^{* * *}$ & $.37 * *$ \\
\hline 11. Orientation & & 1.00 & $.52 * * *$ & $.34 *$ & $.43 * *$ & $.50 * * *$ & $.33^{*}$ & $.39 * *$ \\
\hline 12. Language & & & 1.00 & $.57 * * *$ & $.58 * * *$ & $.77 * * *$ & $.72 * * *$ & $.77 * * *$ \\
\hline 13. Attention & & & & 1.00 & $.43^{* *}$ & $.58 * * *$ & $.46^{* * *}$ & $.39 * *$ \\
\hline 14. Praxis & & & & & 1.00 & $.56 * * *$ & $.38^{* *}$ & $.44 * *$ \\
\hline 15. Visuospatial & & & & & & 1.00 & $.52 * * *$ & $.56^{* * *}$ \\
\hline 16. Construction & & & & & & & 1.00 & $.69 * * *$ \\
\hline 17. Orientation to Name & & & & & & & & 1.00 \\
\hline
\end{tabular}

Note: TMT $=$ Trail Making Test (Reitan \& Wolfson, 1985). DSF = Digit Span Forward (Wechsler, 2008). CDT = Clock Drawing Test (Mendez, Ala, \& Underwood, 1992). SIB = Severe Impairment Battery (Saxton, McGonigle-Gibson, Swihart, \& Miller, 1990).

QUALID = Quality of Life in Late Stage Dementia (Weiner et al., 2000). ADFACS = Alzheimer's Disease Functional Assessment and Change Scale; IADL = Instrumental Activities of Daily Living; ADL = Activities of Daily Living (Manero et al., 2014). M-NCAS $=$ Modified Nursing Care Assessment Scale (Kleinman, Frank, Ciesla, Rupnow, \& Brodaty, 2004). ${ }^{*} p<.05 .^{* *} p<.01 .{ }^{* * *} p<.001$. 
values for the TMT was significantly associated with greater impairment in IADLs $(r=.28, p$ $=.041)$ and $\operatorname{ADLs}(r=.29, p=.030)$.

\section{Post hoc Analysis: Correlations Between Psychotropic Medications, Medical Comorbidity Ratings, and Study Variables; Controlling for SIB Total Score}

In the above models, patients' medication use and comorbid medical diagnoses were not included as potential predictor variables. However, these factors may be important considerations as they could impact the outcome variables examined in this study. Therefore, post hoc point biserial and Pearson's correlations were conducted to determine whether psychotropic medication use and comorbid medical diagnoses significantly correlated with our main study variables. Results of these analyses are presented in Table 15. Psychotropic medications were examined in the sample, which revealed that 41 participants $(76.4 \%)$ were on acetylcholine esterase inhibitors, 12 participants $(21.8 \%)$ were on antipsychotics, 6 participants (10.9\%) were on benzodiazepines, and 24 participants (43.6\%) were on antidepressants. Patients' medical comorbidities were categorized using the Charlson Comorbidity Index (for a review of this index see Charlson, Pompei, Ales, \& MacKenzie, 1987; Quan et al., 2005; Quan, Parsons, \& Ghali, 2002). The mean score on this index was 2.84 and the standard deviation was 1.68 . The rationale for the post hoc examination of psychotropic medications and the use of the Charlson Comorbidity Index is described in the discussion below.

Patients' ratings on the Charlson Comorbidity Index was not found to be significantly correlated with CFs or any of the current study's outcome variables. The use of antidepressant medication was significantly associated with the presence of greater disruptive behaviours or characteristics, as measured by the M-NCAS Attitude score, $r=.30, p=.026$. Also, the use of antidepressant medication was significantly associated with greater impairment in IADLS, as 
Table 15

Variable Intercorrelations for Medication Use and Charlson Comorbidity Index Rating

\begin{tabular}{|c|c|c|c|c|c|c|}
\hline & 1 & 2 & 3 & 4 & 5 & 6 \\
\hline 1. Charlson Comorbidity Index & 1.00 & .02 & $-.35 *$ & .07 & -.11 & -.20 \\
\hline 2. Acetylcholine Esterase Inhibitors ${ }^{1}$ & & 1.00 & -.02 & -.08 & .14 & .10 \\
\hline 3. Antipsychotics ${ }^{1}$ & & & 1.00 & -.04 & .25 & .09 \\
\hline 4. Benzodiazepines ${ }^{1}$ & & & & 1.00 & $-.31 *$ & -.18 \\
\hline 5. Antidepressants ${ }^{1}$ & & & & & 1.00 & .10 \\
\hline 6. DCFS & & & & & & 1.00 \\
\hline 7. SIB Total Score & & & & & & \\
\hline 8. M-NCAS Attitude Total & & & & & & \\
\hline 9. M-NCAS Strain Total & & & & & & \\
\hline 10. ADFACS IADL & & & & & & \\
\hline 11. ADFACS ADL & & & & & & \\
\hline 12. QUALID & & & & & & \\
\hline
\end{tabular}

Note: DCFS = Dementia Cognitive Fluctuation Scale (Lee et al., 2013). SIB = Severe Impairment Battery (Saxton, McGonigle-Gibson, Swihart, \& Miller, 1990). M-NCAS = Modified Nursing Care Assessment Scale (Kleinman, Frank, Ciesla, Rupnow, \& Brodaty, 2004). ADFACS = Alzheimer's Disease Functional Assessment and Change Scale; IADL $=$ Instrumental Activities of Daily Living; ADL = Activities of Daily Living (Manero et al., 2014). QUALID = Quality of Life in Late Stage Dementia (Weiner et al., 2000). ${ }^{1}$ Point-biserial correlation. ${ }^{*} p<.05 .{ }^{* *} p<.01 .{ }^{* * * *} p<.001$. 
Table 15 (continued)

Variable Intercorrelations for Medication Use and Charlson Comorbidity Index Rating

\begin{tabular}{|c|c|c|c|c|c|c|}
\hline & 7 & 8 & 9 & 10 & 11 & 12 \\
\hline 1. Charlson Comorbidity Index & .15 & .09 & -.13 & -.04 & .04 & $\begin{array}{l}-.02 \\
\end{array}$ \\
\hline 2. Acetylcholine Esterase Inhibitors ${ }^{1}$ & .11 & -.12 & -.14 & -.06 & -.13 & -.21 \\
\hline 3. Antipsychotics ${ }^{1}$ & -.15 & .03 & .14 & .19 & .08 & .14 \\
\hline 4. Benzodiazepines ${ }^{1}$ & .15 & .04 & -.02 & -.14 & -.17 & .09 \\
\hline 5. Antidepressants ${ }^{1}$ & -.23 & $.30 *$ & .24 & $.34 *$ & .06 & .17 \\
\hline 6. DCFS & $-.31 *$ & $.31 *$ & $.33 *$ & .12 & $.45^{* *}$ & $.29 *$ \\
\hline 7. SIB Total Score & 1.00 & -.13 & -.06 & $-.35^{*}$ & $-.56^{* *}$ & -.02 \\
\hline 8. M-NCAS Attitude Total & & 1.00 & $.72 * * *$ & $.43 * *$ & $.38 * *$ & $.55^{* * *}$ \\
\hline 9. M-NCAS Strain Total & & & 1.00 & .12 & .14 & $.43 * *$ \\
\hline 10. ADFACS IADL & & & & 1.00 & $.60 * * *$ & $.29 *$ \\
\hline 11. ADFACS ADL & & & & & 1.00 & .26 \\
\hline 12. QUALID & & & & & & 1.00 \\
\hline
\end{tabular}

Note: DCFS = Dementia Cognitive Fluctuation Scale (Lee et al., 2013). SIB = Severe Impairment Battery (Saxton, McGonigle-Gibson, Swihart, \& Miller, 1990). M-NCAS = Modified Nursing Care Assessment Scale (Kleinman, Frank, Ciesla, Rupnow, \& Brodaty, 2004). ADFACS = Alzheimer's Disease Functional Assessment and Change Scale; IADL $=$ Instrumental Activities of Daily Living; ADL = Activities of Daily Living (Manero et al., 2014). QUALID = Quality of Life in Late Stage Dementia (Weiner et al., 2000). ${ }^{1}$ Point-biserial correlation. ${ }^{*} p<.05 .{ }^{* *} p<.01 .{ }^{* * * *} p<.001$. 
measures by the ADFACS-IADL score, $r=.34, p=.012$. No other significant correlations between study outcome variables and patients' use of psychotropic medications and medical comorbidity ratings were found.

Finally, post-hoc multiple hierarchical regressions were run with patients' SIB Total Score added to Step 1 to determine if the same pattern of results occurs when controlling for patients' level of cognitive function. The results of these analyses are summarized in Table 16. For all analyses, the pattern of results remained the same when level of cognitive function was added as an additional predictor variable. 
Table 16

Changes in Hierarchical Regression Models When 'SIB Total Score' is Added to Step 1 of Regression $(N=55)$.

$B \quad S E \quad \beta$

Model

\begin{tabular}{|c|c|c|c|}
\hline \multicolumn{4}{|l|}{ ADFACS_ADL } \\
\hline \multicolumn{4}{|l|}{ Step 1} \\
\hline Constant & 54.30 & 23.51 & \\
\hline Education & 0.15 & 0.17 & 0.10 \\
\hline Age at testing & -0.25 & 0.26 & -0.11 \\
\hline Months in LTC & -0.07 & 0.05 & -0.19 \\
\hline SIB Total Score & -0.25 & 0.05 & $-0.55 * * *$ \\
\hline \multicolumn{4}{|l|}{ Step 2} \\
\hline Constant & 39.85 & 23.01 & \\
\hline Education & 0.02 & 0.17 & 0.02 \\
\hline Age at testing & -0.18 & 0.24 & -0.08 \\
\hline Months in LTC & -0.08 & 0.04 & -0.19 \\
\hline SIB Total Score & -0.21 & 0.05 & $-0.45 * * *$ \\
\hline DCFS Total Score & 0.64 & 0.25 & $0.30 *$ \\
\hline \multicolumn{4}{|l|}{ ADFACS_IADL } \\
\hline \multicolumn{4}{|l|}{ Step 1} \\
\hline Constant & 70.50 & 40.17 & \\
\hline Education & -0.70 & 0.30 & -0.31 \\
\hline Age at testing & -0.34 & 0.44 & -0.10 \\
\hline Months in LTC & -0.10 & 0.08 & -0.17 \\
\hline SIB Total Score & -0.24 & 0.09 & $-0.34 *$ \\
\hline \multicolumn{4}{|l|}{ Step 2} \\
\hline Constant & 68.15 & 41.83 & \\
\hline Education & -0.09 & 0.31 & -0.40 \\
\hline Age at testing & -0.33 & 0.44 & -0.10 \\
\hline Months in LTC & 0.10 & 0.08 & -0.17 \\
\hline SIB Total Score & -0.23 & 0.10 & $-0.33^{*}$ \\
\hline DCFS Total Score & 0.10 & 0.45 & 0.03 \\
\hline \multicolumn{4}{|l|}{ QUALID } \\
\hline \multicolumn{4}{|l|}{ Step 1} \\
\hline Constant & 35.52 & 25.33 & \\
\hline Age at testing & -0.17 & 0.28 & -0.09 \\
\hline Months in LTC & -0.07 & 0.05 & -0.19 \\
\hline SIB Total Score & -.01 & 0.06 & 0.02 \\
\hline \multicolumn{4}{|l|}{ Step 2} \\
\hline Constant & 21.07 & 25.10 & \\
\hline Age at testing & -0.12 & 0.27 & -0.06 \\
\hline Months in LTC & -0.08 & -.05 & -0.21 \\
\hline SIB Total Score & 0.04 & 0.06 & 0.09 \\
\hline DCFS Total Score & 0.60 & 0.26 & $0.32 *$ \\
\hline MNCAS Attitude & & & \\
\hline
\end{tabular}




\begin{tabular}{|c|c|c|c|}
\hline \multicolumn{4}{|l|}{ Step 1} \\
\hline Constant & 133.13 & 53.75 & \\
\hline Age at testing & -0.77 & 0.58 & -0.18 \\
\hline Months in LTC & -0.18 & 0.10 & -0.23 \\
\hline SIB Total Score & -0.11 & 0.12 & -0.12 \\
\hline \multicolumn{4}{|l|}{ Step 2} \\
\hline Constant & 103.14 & 53.38 & \\
\hline Age at testing & -0.66 & 0.56 & -0.15 \\
\hline Months in LTC & -0.20 & 0.10 & -0.25 \\
\hline SIB Total Score & -0.02 & 0.12 & -0.02 \\
\hline DCFS Total Score & 1.25 & 0.55 & $0.31 *$ \\
\hline \multicolumn{4}{|l|}{ MNCAS Strain } \\
\hline \multicolumn{4}{|l|}{ Step 1} \\
\hline Constant & 117.38 & 52.53 & \\
\hline Age at testing & -0.72 & 0.57 & -0.17 \\
\hline Months in LTC & -0.09 & 0.10 & -0.12 \\
\hline SIB Total Score & -0.05 & 0.12 & -0.06 \\
\hline \multicolumn{4}{|l|}{ Step 2} \\
\hline Constant & 85.42 & 51.66 & \\
\hline Age at testing & -0.61 & 0.55 & -0.15 \\
\hline Months in LTC & -0.10 & 0.10 & -0.14 \\
\hline SIB Total Score & 0.05 & 0.12 & 0.05 \\
\hline DCFS Total Score & 1.34 & 0.54 & $0.34 *$ \\
\hline \multicolumn{4}{|c|}{$\begin{array}{l}\text { Note. } * p<.05 . * * p<.01 . * * * p<.001 . \text { LTC }=\text { Long term care. DCFS = Dementia Cognitive } \\
\text { Fluctuation Scale (Lee et al., 2013). ADFACS = Alzheimer's Disease Functional Assessment } \\
\text { and Change Scale (Manero et al., 2014); ADL = Activities of Daily Living; IADL = Instrumental } \\
\text { Activities of Daily Living; QUALID = Quality of Life in Late Stage Dementia (Weiner et al., } \\
\text { 2000); M-NCAS = Modified Nursing Care Assessment Scale (Kleinman, Frank, Ciesla, Rupnow, } \\
\text { \& Brodaty, 2004) }\end{array}$} \\
\hline
\end{tabular}




\section{Discussion}

The present study sought to determine whether the presence and severity of CFs predicts a series of functional features in a sample of individuals with dementia living in a long-term care facility. It was hypothesized that CFs, as measured by the DCFS (Lee, McKeith, Mosimann, Ghosh-Nodial, et al., 2013), would predict greater impairment on neuropsychological function. In addition, it was hypothesized that CFs would predict degree of nurse-reported caregiver strain, as well as patients' ability to complete activities of daily living and affect their general quality of life.

Hierarchical multiple regression analyses largely supported these hypotheses. A model emerged predicting neuropsychological test performance, as measured by the SIB, based on the severity of patients' CFs. Similar models emerged with CFs predicting scores on measures of activities of daily living, as measured by the ADFACS, quality of life, as measured by the QUALID, and nurse strain, as measured by the M-NCAS. Importantly, post-hoc analyses suggest that these patterns hold true even when accounting for patients' level of cognitive function.

\section{CFs and Level of Cognitive Functioning}

The first aim of this study was to examine the association between CFs and cognitive test performance. Specifically, this study sought to determine whether patients' scores on the DCFS predicted performance on the SIB, a measure designed to assess cognitive function in patients with advanced-stage dementia. Consistent with Study Aim 1a hypotheses, the severity of CFs significantly predicted cognitive functioning in that greater severity of CFs predicted poorer cognitive functioning in the areas of orientation, language, and praxis. Similar findings have been demonstrated in other studies that tested the association between CFs and 
neuropsychological performance; however, these studies used different assessment measures and focused on community-dwelling individuals with less severe dementia.

Escandon et al. (2010) examined the association between CFs and cognitive function in a sample of community-dwelling individuals with AD. Similar to the findings of the current study, the researchers found that the presence of CFs was associated with significantly reduced performance on measures of cognitive function. Specifically, they found an inverse correlation between the presence of CFs and composite scores reflecting global function, memory, visuospatial skills, and working memory, with the presence of CFs explaining $3-11 \%$ of the variance in performance. The authors also found significant inverse correlations between the presence of CFs and individual neuropsychological measures. Among these, the authors report that poorer performance on measures of verbal fluency and confrontation naming was correlated with the presence of CFs. Similarly, the current study found that CFs predicted language subtest scores on the SIB (Panisset et al., 1994); however, unlike Escandon et al.'s (2010) study, the current study found that CFs did not predict memory or visuospatial subtest scores. There are several possible explanations for the differing pattern of associations observed between studies.

First, Escandon et al. (2010) assessed for the presence of CFs using a modified four-item version of the MCFS. This version requires informants to rate patients by responding yes or no to the following four questions: "1) drowsiness and lethargy all the time or several times a day despite getting enough sleep the night before (daytime somnolence); 2) daytime sleep of 2 or more hours before 7 PM (sleeps $>2$ hours); 3) times when the patient's flow of ideas seems disorganized, unclear or not logic (illogical, disorganized thinking); and 4) staring into space for long periods (staring spells)." The authors grouped patients based on the number of affirmative responses provided to these items. Those patients with affirmative informant-based responses to 
three or four of the items were categorized as having CFs. Those with two or fewer affirmative responses were categorized as not having CFs. While this method has been demonstrated to effectively differentiate patients with and without CFs in previous studies (Ballard et al., 2001; Varanese, 2010), this approach fails to account for the severity of the fluctuations. The DCFS accounts for this shortcoming by requiring informant-based indications of the severity of each specific symptom assessed by each of the four questions. As a result, the research question examined by Escandon et al. (2010) reflected group differences in cognitive test performance between those patients who experience, and those who do not experience, CFs. In contrast, the current study placed emphasis on the severity of CF symptoms rather than grouping patients based on their presence. This approach was favoured for this study because our sample was comprised primarily of patients with moderate to severe dementia. Previous research has demonstrated that the presence and severity of CFs increases with increasing dementia severity (Escandon et al., 2010) and, thus, we predicted that the majority of our sample would demonstrate at least some degree of CFs. This prediction was supported by the finding that only two patients in our sample were rated as having no CFs (i.e., a score of 5/20 on the DCFS. This difference could certainly account for the differences in outcome between the current study and the Escandon et al. (2010) study.

Second, Escandon et al. (2010) assessed patients' general level of cognitive function using a selection of measures that were normed using "normal adults" or "healthy older adults." Such measures included tests from well-known batteries such as the Wechsler Memory Scale (WMS; Wechsler, 1993) and the Wechsler Adult Intelligence Scale (WAIS; Wechsler, 1993). These measures provided a comprehensive assessment that was suitable for their sample, which was comprised almost entirely of individuals with "very mild" dementia ratings on the CDR. 
However, previous research has demonstrated that the progression of $\mathrm{AD}$ and related dementias invariably results in global and severe impairment across all spheres of cognitive functioning. Therefore, as dementia progresses, many neuropsychological and mental status assessments have only limited ability to measure cognitive functions because many patients perform at floor levels (Schmitt et al., 1997). In addition, a major difficulty in measuring cognition in moderate to severe stages of dementia is the continued dissolution of expressive and receptive language skills (Panisset et al., 1994). For this reason, the current study used the SIB (Panisset et al., 1994) to assess global cognitive function because this measure relies on tasks that minimize the need for expressive language skills and uses gestures, pointing, and limited verbalizations for patient responses (Panisset et al., 1994). While this measure has been proven to be a valid measure of cognitive function in patients with moderate to severe dementia (Ferris, Karantzoulis, Somogyi, \& Meng, 2013; Pélissier, Roudier, \& Boller, 2001; Schmitt et al., 1997), it does differ qualitatively from the measures used by Escandon et al. (2010). Thus, it is difficult to determine whether SIB subscales (i.e., memory, language, etc.) can be directly compared to tests designed to measure similar constructs but that rely more heavily on expressive language skills. Further research is needed to confirm the concurrent validity of the SIB, as it is possible that the observed differences between the current study and Escandon et al. (2010) may be due to differences in the underlying cognitive abilities assessed by the measures used in each study.

Third, as mentioned, the sample examined by Escandon et al. (2010) was comprised primarily of patients with "very mild" dementia, with a very small percentage $(16 \%)$ of their sample being classified as having "mild" dementia. No cases were identified as having moderate or severe dementia. As previously mentioned, impairments in cognitive function become more global as dementia progresses (Shuster, 2000). For this reason, differential diagnosis of dementia 
types (e.g., AD vs. DLB) can become more challenging in patients with late stage dementia because global functioning declines to a point where symptom profiles become more similar across dementia types (Folstein, 1997). No studies have specifically investigated whether the relationship between CFs and profiles of cognitive impairment change with increasing dementia severity. One potential factor that could explain the different profiles of cognitive deficits associated with CFs observed in the current study versus the Escandon et al. (2010) study is the difference in dementia severity between the study samples, at is possible that the relationship between CFs and cognitive ability changes as a function of dementia severity. Thus, future longitudinal studies are needed to explore potential characteristic differences between CFs in early versus late-stage dementia.

Similar issues arise when attempting to draw comparisons between the current study and that of Varanese et al. (2010). In their study, the authors compared performance on cognitive test measures between patients with Parkinson's disease and no associated CFs to those with Parkinson's disease with associated CFs. The authors used the CAF and classified patients with a score of 3/6 as possessing CFs. Thus, similar to the study by Escandon et al. (2010), the severity of the CFs was not considered in this study. Consistent with the findings of the current study, Varanese et al. (2010) found that the presence of CFs was associated with significantly reduced performance on a global measure of cognitive function. Specifically, the authors found that, compared to patients without CFs, those with CFs had significantly lower Attention, Initiation and Perseveration, and Memory subscores on the Dementia Rating Scale - Version 2 (DRS-2; Jurica, Leitten, \& Mattis, 2001). The results of this study further support the importance of CFs in predicting cognitive test performance, but the pattern of deficits differs from those reported by Escandon et al. (2010) and the findings of the current study. Potential contributing factors 
accounting for these differences again include differences in dementia type and severity, as well as different measures for assessment of $\mathrm{CFs}$ and cognitive function.

Interestingly, when degree of variability in cognitive test performance across three administrations during a one-week period was used as the measure of CFs in the current study, the pattern of cognitive deficits associated with greater severity of CFs matched the findings of previous studies. Specifically, greater variability in DSF performance was associated with poorer performance on the Memory, Attention and Visuospatial subscales of the SIB, which is a pattern very similar to the deficits associated with the presence of CFs identified by Escandon et al. (2010) and Varanese et al. (2010). In addition, the current study found that greater variability in CDT and TMT performance was associated with significantly poorer performance on the Attention, Orientation, and Praxis subscales of the SIB. Of note is that participants' orientation and praxis abilities were not assessed in the Escandon et al. (2010) and Varanese et al. (2010) studies and therefore comparison of performance in these domains across studies is not possible. Importantly, variability in test performance across three trials was not found to be significantly associated with DCFS scores in the current study. This may suggest that the underlying constructs assessed by the DCFS differ from those captured by the MCFS and CAF, and therefore the DCFS is not a good predictor of short-term variability on brief cognitive tests.

Further evidence that the underlying constructs assessed by the DCFS may differ from those assessed by the DCFS' predecessors (i.e., MCFS and CAF) is found when drawing comparisons to previous research that similarly implemented the use of variability in cognitive test performance across multiple time points as a measure of CFs. For example, Ballard et al. (2001) examined a sample of 30 dementia sufferers (15 AD, 15 DLB) and found that CFs, as measured by the CAF, were associated with variability in cognitive test performance across three 
trials within a period of one week. Specifically, they found that participants with identified CFs demonstrated more variable performance when their functioning was monitored over time using the cognitive drug research (CDR) computerized neuropsychological test battery (Simpson, Surmon, Wesnes, \& Wilcock, 1991). Measures of attention, reaction time, and vigilance were reported to be most strongly associated with CAF scores. This finding supports the current study's conclusions that CFs can be detected using simple cognitive measures administered over multiple trials. In addition, this variability in performance is significantly positively associated with scores on the informant-based CAF. Further research is needed to determine if the lack of an association between the DCFS and cognitive test variability observed in the current study is due to differences between studies in the cognitive test measures used, or if the difference is attributable to a dissimilarity in underlying constructs assessed by the DCFS compared to its predecessors.

In addition to cognitive performance at the time of the assessment, the current study also examined retrospective data from the CPS of the MDS (Morris et al., 1994) to determine if the presence of CFs is associated with a recent decline in cognitive functioning (Study Aim 1b). When assessed using the DCFS, CFs were not associated with a significant change in CPS scores. However, greater CFs as measured by variability in CDT performance over a one-week period was significantly associated with greater decline in CPS scores. This suggests that short-term fluctuations on the CDT may represent an indicator of recent changes in cognitive function, which in turn may assist in identifying patients whose care needs are actively changing.

In summary, CFs appear to be associated with cognitive test performance, with more severe CFs predicting greater cognitive impairment. However, when compared to previous studies, the current results suggest that the pattern of cognitive deficits associated with the 
presence of CFs and the ability to predict retrospective decline in cognitive function differs depending on the method used to identify them. A higher rating on the DCFS is associated with poorer performance on measures of language, orientation, and praxis. This profile of cognitive deficits differs from studies that measured CFs using the MCFS and CAF. This difference is unexpected given that the DCFS was developed to incorporate the best features of both the MCFS and CAF into an easy-to-administer measure. However, these differences could be related to different patient samples and cognitive test measures used across studies.

\section{CFs and Activities of Daily Living}

The second aim of the present study was to examine the association between CFs and patients' ability to engage in activities of daily living. Specifically, this study sought to determine whether patients' scores on the DCFS predicted scores on the ADFACS, a measure designed to assess both ADLs and IADLs (Manero et al., 2014). Consistent with Study Aim 2a hypotheses, increased severity of CFs significantly predicted greater impairment in patients' ability to perform ADLs. This finding is consistent with Ballard et al. (2001) who, to the author's knowledge, completed the only other study examining this relationship and similarly found a significant association between the presence of CFs and patients' ability to perform ADLs.

Contrary to preliminary hypotheses for Study Aim 2b, patients' scores on the DCFS failed to significantly predict IADLs. A possible explanation for this unexpected result may relate to a scoring issue created by a combination of the measure's scoring protocol and the setting that the data was collected in. Since the current sample was collected from an inpatient veteran's long-term care facility, all of the participants lived in units that were attended by nurses and other hospital staff. In this facility, patients' meals and medications are prepared for them and if patients choose to go shopping in the community they are usually accompanied by family 
or other caregivers. As a result, patients are not required to complete many of the IADLS that seniors living in the community would engage in on a regular basis. The ADFACS response form allows informants to indicate "not assessable" when a certain item (e.g., "does the patient prepare meals for him/herself") cannot be assessed. When scoring, any items that received a "not accessible" rating are replaced with the patients' average score on all remaining items. In the current study, this meant that some patient's had multiple IADL items rated as "not assessable" simply because their living situation does not require them to complete certain tasks. Thus, the total IADL score for some patients was based on the average of the few IADL-specific items that did receive ratings. For obvious reasons, this may have influenced the quality of the data collected by this specific portion of the ADFACS measure.

To date, no study has examined the relationship between ADLs and variability in cognitive test performance across multiple trials. As mentioned previously, the current study included a secondary measure of CFs that was based on variability in performance on the DSF, CDT, and TMT administered across three separate trials. Interestingly, we found that variability on the CDT and TMT was significantly associated with ADFACS ADL scores, as well as IADL scores. This seems to suggest that certain characteristics of CFs may in fact be associated with IADLs, even in patients with moderate to severe dementia who are living in an inpatient facility. However, this finding needs to be interpreted with caution since, as mentioned, the IADL portion of the ADFACS contains items that were not relevant to some study participants, which reduced the validity of this measure.

Although very limited research to date has examined the relationship between CFs and ADLs, the current findings are consistent with Ballard et al. (2001) and provide further support for the importance of CFs in predicting patients' ability to effectively complete basic self-care 
tasks. Furthermore, results of the current study suggest that CFs may also play an important role in determining patients' ability to engage in activities that may let an individual live independently in a community, such as basic housework, preparing meals, and taking medications as prescribed.

\section{CFs and Quality of Life}

The third aim of this study was to examine the association between CFs and patients' overall quality of life. Specifically, this study sought to determine whether patients' scores on the DCFS predicted scores on the QUALID, a valid and reliable instrument for rating quality of life in persons with late-stage $\mathrm{AD}$ and other dementing illnesses (Weiner et al., 2000). Consistent with Study Aim 3 hypotheses, increased severity of CFs significantly predicted lower overall quality of life. Although previous research has suggested that individuals with DLB experience more severe CFs and also report lower quality of life than individuals with other forms of dementia (Boström, Jonsson, Minthon, \& Londos, 2007), to the author's knowledge, no study to date has examined the impact of CFs on quality of life in patients with dementia.

In their study of community-dwelling patients with mild to moderate dementia, Boström et al. (2007) found that a DLB diagnosis predicted an almost 3-fold increase in resource utilization and a significantly lower quality of life compared to AD. The authors identified a number of contributors to poorer quality of life in their sample, which included a greater presence of behavioural disturbances, lower independence in IADLs, and the presence of apathy and delusions. The current study hypothesized that another potential contributing factor to lower quality of life in patients with DLB could be the presence of more frequent and severe CFs.

Although the current study did not include patients with DLB, the presence of CFs in the sample was still significant. This is most likely due to the sample being comprised of patients 
with moderate to severe dementia and, as mentioned previously, the rate and severity of CFs increases with increasing dementia severity (Escandon et al., 2010). The finding that increasing severity of CFs predicts lower quality of life ratings, even when the effects of age and length of time in long-term care is accounted for, provides support for the earlier hypothesis that CFs may be accounting for quality of life differences seen in early DLB. Of course, this hypothesis requires further investigation in group-based research studies. The inclusion of a CFs rating system in a study similar to the one conducted by Boström et al. (2007) may help to better characterize differences between dementia types that contribute to greater declines in quality of life.

\section{CFs and Nursing Strain}

The fourth and final aim of the current study was to examine the association between the severity of patients' CFs and caregiver distress. To explore this relationship, the present study sought to determine whether patients' scores on the DCFS predicted scores on the M-NCAS, a valid and reliable means of obtaining care burden ratings from formal caregivers in long-term care (Kleinman et al., 2004). Consistent with Study Aim 4 hypotheses, increased severity of CFs significantly predicted increased self-reported strain in patients' primary nurses. This finding is important, as the provision of care for institutionalized patients with dementia is known to be a daunting task that is often associated with substantial strain and burnout (Beck et al., 1999;

Kleinman et al., 2004; Mahoney et al., 1999), but there remains a scarcity of research examining this issue. To date, only one other study has explored the specific impact of CFs on burden in caregivers of persons with dementia (Lee, McKeith, Mosimann, Ghosh-Nodial, et al., 2013). In their study, the authors also used the DCFS as an assessment of CFs and, similar to the present study, they found a significant association between DCFS scores and caregiver burden. They 
concluded that the emergence of CFs is associated with a significantly increased level of burden for the caregiver. Furthermore, the authors stressed the need for further work in this area, particularly considering the increased rate at which carers in their study reported the occurrence of CFs. Although the general findings of their study match well with the current study, there are important methodological differences to consider. First, Lee et al. (2013) collected an outpatient sample of patients with dementia and the patients' caregivers were typically their spouses or children. Therefore, the relationship between CFs and caregiver burden in an institutionalized setting has not been previously examined. As mentioned, the current study found that increasing severity of CFs predicts greater self-reported strain in patients' primary nurses. This finding adds further support to the notion that CFs are of critical importance when considering factors associated with caring for patients with dementia. In addition, this finding sheds light on a potential contributing factor to the high rate of nursing turnover that is currently impacting health care in Canadian long-term care facilities (Chu et al., 2013).

In their study, Lee et al. (2013) acknowledged that their use of the CDS from NPI limited their ability to characterize the relationship between CFs and carer distress. This is because the CDS focuses primarily on symptoms related to mood and psychotic symptoms, which reduced the study's ability to adequately examine the contribution of factors other than neuropsychiatric symptoms (i.e., mood and psychotic symptoms). Therefore, the current study sought to more accurately identify the association between CFs and caregiver distress by using a more comprehensive measure of nurse-reported caregiver strain. In so doing, we found patients with more severe CFs were rated as possessing lower autonomy and predictability. In addition, patients with more severe CFs were perceived by caregivers to have more dysfunctional affect. Importantly, patients with more severe CFs were reported to provide less job satisfaction by their 
caregiver, which presumably reflects the combined influence of the above-mentioned factors (i.e., autonomy, predictability, and affect).

Although current research examining the relationship between CFs and caregiver strain is sparse, especially among inpatient populations, there appears to be support for the importance of CFs in predicting caregiver strain. Not only do CFs impact upon the quality of life of spouses and other loved ones, possibly leading to earlier admittance to long-term care facilities, but once admitted to institutionalized care the presence of CFs may negatively impact nursing staff.

\section{Study Implications}

The current study represents a comprehensive examination of the association between CFs and other factors contributing to patients' psychosocial and cognitive wellbeing. The first major implication of this study relates to the conceptualization of CFs in dementia. As highlighted by previous research in this area, the term 'CFs' remains elusive, despite a number of attempts to operationally define and assess the phenomenon (Bradshaw et al., 2004; Ferman et al., 2004; Lee et al., 2013, 2012; McKeith, Perry, Fairbairn, Jabeen, \& Perry, 1992; Walker, Ayre, et al., 2000; Walker, Ballard, et al., 2000). As a result, there remains considerable difficulty in characterizing and assessing the core features of CFs in dementia (Lee et al., 2012; Mega et al., 1996). The accurate identification of CFs represents a major clinical issue as the presence of CFs is considered to be an important distinguishing factor between patients with early-stage DLB versus those with early-stage $\mathrm{AD}$. This differential diagnosis is an important consideration when clinicians are deciding on treatment plans. For example, there is evidence to suggest that patients with DLB respond better to cholinesterase inhibitors than those with $\mathrm{AD}$, and that these patient groups appear to have different disease trajectories (McKeith et al., 2000; Serrano \& GarcíaBorreguero, 2004). 
To better characterize CFs from a cognitive perspective, a primary goal of the current study was to determine the impact of CFs on general cognitive function. Interestingly, the cognitive profile associated with CFs was found to vary greatly depending on the method used to identify CFs. When assessed using the DCFS, as in the current study, increased severity of CFs was found to predict greater impairment on measures of orientation, language, and praxis. In contrast, when measured using the MCFS, the presence of CFs was found to be associated with decreased performance on measures of language, memory, visual spatial ability, and working memory (Escandon et al., 2010). Yet another profile of cognitive deficits emerged when the presence of $\mathrm{CFs}$ is determined using the CAF; specifically, CFs were found to be associated with deficits in attention, initiation and perseveration, and memory (Varanese, 2010). Similar to Ballard et al. (2001) and Walker et al. (2000), the current study sought to further explore the influence of CFs using a more objective assessment of CFs. Correlation of variance scores reflected variability in patients' performance on the TMT, CDT, and DSF tasks. When correlation of variance scores were used as a measure of the severity of CFs, additional patterns of associated performance deficits emerged. Overall, the results of the current study add to the growing literature suggestions that $\mathrm{CFs}$ are associated with a general decline in cognitive test performance. However, the specific pattern of cognitive deficits remains unclear and highly dependent on the specific method used to detect CFs.

The second major implication of this study relates to the clinical importance of identifying individuals who are experiencing CFs. This study found that CFs exert a broad influence over other aspects of patients psychosocial wellbeing. Specifically, CFs were found to significantly predict a decreased ability to engage in activities of daily living, as well as lower reported quality of life. These specific associations have been alluded to in previous research, but few studies 
have directly examined this in patient populations and no previous research has explored these associations in a sample of patients residing in a long-term care facility. These findings are clinically important because they suggest that patients with more severe CFs should be flagged as individuals who will likely require a higher level of care and assistance with completion of self-care tasks (e.g., bathing, feeding, etc.). For individuals with dementia who continue to live in the community or who have entered institutionalized care, the early presence of CFs could alert clinicians to the increased risk of developing psychological and behavioural issues that lead to a reduction in the patient's quality of life. This knowledge is important when working with patients at all stages of dementia because it could help guide decisions about entry into various targeted therapeutic programs for patients and their families.

A third major implication of this study relates to the finding that CFs impact nurses' selfreported burden or strain. As mentioned previously, nursing staff turnover in long-term care homes has been attributed to a variety of factors, including organization leadership practices and behaviours, supervisory support, burnout, job satisfaction, and work environment satisfaction (Chu et al., 2013).

Taken together, these findings suggest that CFs are associated with a significant amount of caregiver strain, which may partially explain high turn-over rates of nursing staff in inpatient clinics. From an administrative perspective, identifying which patients experience CFs may help with staffing decisions and patient placement planning. In addition, now that this relationship has been identified, methods of training patient care staff could potentially be implemented that accommodate for cognitive fluctuations. Movement towards a greater recognition of the importance of accommodating for fluctuations when caring for patients in inpatient facilities has already begun. Guidelines published by the British National Collaborating Centre for Mental 
Health provide information on supporting people with dementia and their carers in health and social care (National Collaborating Centre for Mental Health, 2007). In these guidelines, the importance of including "flexibility to accommodate fluctuating abilities" is highlighted as a method of promoting and maintaining independent activity, as well as helping patients to enhance function, adapt and develop skills, and minimize the need for support. Of course, such flexibility adds to the complexity of caring for patients in inpatient settings, especially in settings comprised of patients with highly variable care needs. However, identifying which patients experience CFs could play an important role in developing individualized care plans for dementia sufferers.

\section{Study Limitations}

First, as mentioned in the study methods, the DCFS had poor internal reliability in the current study. Cronbach's alpha is used to represent a coefficient of reliability (or consistency) of a psychometric test. Most statistical guidelines suggest that reliable measures should be characterized by Cronbach's alpha values equal to or greater than .70 (DeVellis, 2003; Field, 2005; Tavakol \& Dennick, 2011). The Cronbach's alpha for the DCFS was found to be .52, which falls in the "poor" range. Fortunately, reliability scores for all other study measures were at or above the acceptable level, with the majority falling in the "excellent" range (i.e., Cronbach's alpha greater than .90). Although the reliability of the DCFS appears questionable, it is important to consider how the alpha value is calculated. The calculation for Cronbach's alpha assumes that each test item measures the same latent trait on a scale. Therefore, if multiple factors/traits underlie the items on a scale, the assumption is violated and alpha underestimates the reliability of the test (Tavakol \& Dennick, 2011). As mentioned previously, 'CFs' is a broad term that remains poorly characterized. In an attempt to facilitate the identification of CFs, Lee et 
al. (2013) developed a research version based on four items from their original scale that loaded onto three factors. Therefore, the authors knowingly created a scale based on multiple factors/traits because these four items were found to show good levels of sensitivity (range: 78.6\%-80.3\%) and specificity (range: 73.9\%-79.3\%) in detecting CFs. Another explanation for the lower-than-anticipated Cronbach's alpha score relates to the fact that the DCFS is comprised of only four items, and alpha is known to underestimate the reliability of a scale when the number of scale items is small (Tavakol \& Dennick, 2011).

A second limitation of the current study concerns the use of the same versions of the TMT, CDT, and DSF for each of the three test administrations over the one-week observation period. This procedure raises concern about the potential confounding effect of practice effects and whether this may influence the study's findings. Previous research has suggested that practice effects on tests administered to patients with dementia tend to be negligible, especially as the disease progresses into late stages (Goldberg, Harvey, Wesnes, Snyder, \& Schneider, 2015). Nonetheless, these potential effects are important methodological considerations regardless of the nature of the participant sample. In deciding whether or not to use multiple variants of these measures across trials, several considerations were made. First, the purpose of the inclusion of these measures was to explore variability in performance, rather than using these measures to track patients' best possible performance in a one-week period. Therefore, even if practice effects were to emerge, we would still expect to see more variability in performance in patients with CFs versus those without. However, a conceivable outcome could be that practice effects occur differentially in those with CFs versus those without. For this reason, future research should explore the relationship between CFs and practice effects. Second, although the CDT has a plethora of scoring options, the production of variants for use in serial assessments is 
made challenging by the fact that the "time setting" criteria is the only aspect of the task that can be altered. Therefore, practice effects are likely to occur on this task even when multiple versions are used.

It is important to note, however, that the use of test variants to avoid practice effects has been challenged by some researchers who suggest that changing subtle features of a test, such as the placement of stimuli on a page, does little to reduce the effect. Previous research suggests that although alternate test forms have been shown to minimize practice effects on tests of declarative memory and spatial processing (Fastenau, Hankins, McGinnis, Moy, \& Richard, 2002; Zgaljardic \& Benedict, 2001), they do little to minimize these effects on procedural tests (Beglinger et al., 2005). In fact, practice effects have been observed in studies involving serial assessment using alternate versions of the DSF (Collie, Maruff, Darby, \& McStephen, 2003) and the TMT (Buck, Atkinson, \& Ryan, 2008). Therefore, any research that incorporates the use of serial testing to assess CFs is likely to be influenced by practice effects; however, it is important to be aware of these effects when interpreting study findings.

A third limitation of the current study is the use of a sample that was comprised of patients with a variety of dementia types. While this sample accurately represents the type of patients currently living in Canadian long-term care facilities, comparisons could not be made across dementia types due to sample size restrictions. As mentioned, previous research has acknowledged that 'CFs' is a term that remains elusive despite several attempts to identify, quantify, and assess the phenomenon (McKeith et al., 2005). While it is known that the frequency and severity of CFs differ depending on dementia subtype (Ballard et al., 2001; Lee et al., 2012), one particular issue concerning the current study is the potential that other qualitative characteristics of CFs also differ depending on dementia subtype. For example, do the 
fluctuations occurring in patients with $\mathrm{AD}$ have a particular quality or set of characteristics that differs from the type of fluctuations seen in patients with DLB? Very recently, this question was examined in a study that used a measure of dysgraphia to determine whether CFs affect test performance differentially in patients with AD versus patients with DLB (Onofri, Mercuri, Donato, \& Ricci, 2015). The authors found a significant association between CFs and handwriting ability in AD that was not present in DLB. This finding suggests that CFs may differentially impact cognitive test performance depending on a patient's particular dementia type. However, more research is needed to determine whether this finding of differential cognitive impairment holds true when using tests assessing a broader range cognitive domains.

A fourth limitation of the current study concerns methodological issues that affect the generalizability of the findings. First, the sample was predominantly male. This was due to the sample being collected from a veteran's long-term care facility, which contains a predominantly male population of patients. Unfortunately, this means that the results and implications of this study need to be interpreted with caution when considering their impact on female patients with dementia, who comprise the majority of residents in most long-term care settings. In addition, because this study involved testing patients directly in their rooms, it was not possible to control for certain variables that may have influenced test performance. For example, some patients were tested in shared room and, on occasion, other staff members were present in the area during testing. However, due to the number of potential contributors present in inpatients settings, attempting to control for all potential variables was not feasible. However, future research could examine individual contributors, such as medications, to determine whether they are associated with the presence or characteristics of CFs in dementia. 
A fifth limitation of the current study concerns the restricted number of variables that were identified and entered into the first step of the various regression analyses. As with all research that is correlational and exploratory in design, it was not possible to control for the wide range of potential variables that could impact participants' performance on cognitive testing and their informant-based scores on the questionnaires. Based on previous research (e.g., Ardila et al., 2000; Ganguli et al., 1991; Wilson et al., 2007), participants' age, level of education, and length of time spent in long-term care were identified as variables that are likely to be associated with the study's main variables of interest. However, the current study did not include variables related to patients' medication or comorbid diagnoses in the model construction. Omitting these variables from the analyses certainly reduces the generalizability of the current findings.

Given that this study was designed to be exploratory in nature it was felt that maintaining focus on several key variables that have been identified in previous studies for their potential impact on patients' CFs, general cognitive function, ability to engage in ADLs and/or QOL was most crucial. Of course, it is not possible to identify and include all potential contributing factors. Due to the sample size available for the current study, care needed to be taken to balance the reduced generalizability created by excluding certain predictive variables with the reduction in statistical power associated with including too many predictive variables. For this reason, the inclusion of variables related to medical comorbidity and medication use was not feasible in the main analyses. Nonetheless, the contribution of medications and comorbidity is an important consideration that should be explored. To accomplish this, the current study conducted post hoc correlational analyses to explore any potential associations between the study's main variables of interest and participants' use of psychotropic medications, as well as their level of medical comorbidity. 
Psychotropic medications were specifically targeted for investigation because previous research suggests that they may interfere with cognitive functioning (for a review see Chew et al., 2008; Moore \& O'Keeffe, 2012). The results of these analyses found that participants who were taking anti-depressant medications were rated as engaging in a significantly greater number of disruptive behaviours on a measure of caregiver burden. In addition, those participants who were taking antidepressant medications were also rated as being less able to complete IADLs. Other types of medications (i.e., benzodiazepines, acetylcholine esterase inhibitors, antipsychotics) were not found to be associated with any of the outcome variables or cognitive fluctuations in the current sample.

Medical comorbidity was assessed using the Charlson Comorbidity Index (Charlson et al., 1987). The Charlson Comorbidity Index is a method of categorizing comorbidities of patients based on the International Classification of Diseases (World Health Organization, 1992) found in administrative data, such as hospital health records. Each comorbidity has an associated weight that is based on the adjusted risk of mortality or resource use and the sum of all the weights results in a single comorbidity score for a patient. A score of zero indicates that no comorbidities were found. Participants' level of medical comorbidity was not associated with any of the outcome variables or cognitive fluctuations in the current sample.

Given that participants' severity of medical comorbidity was not found to be significantly associated with any key variables of interest in the current study, it is unlikely that including this particular variable in the construction of the regression models would have influenced the results of this study. However, as mentioned previously, predictor variables can sometimes be associated with "suppressor effects." Suppressor effects occur when certain variables appear uncorrelated with outcome variables when examined as separate predictors, but may in fact 
change the prediction value of other variables when entered as part of a group of predictors (MacKinnon et al., 2000). Therefore, future research using a larger sample size should more thoroughly investigate the role of patients' comorbid diagnoses and their role in mediating the relationship between CFs and other clinical factors. Also, antidepressant medications were found to interact with some of the variables examined in this study (i.e., caregiver strain and IADLs). As a result, future research should also include an examination of the impact of medication use on CFs, as well as whether certain medications impact the relationship between CFs and other clinical factors.

\section{Future Directions}

In addition to addressing the study limitations highlighted above, there are aspects of CFs that would benefit from future research. One area that has received little attention to date is the long-term outcome associated with the presence of CFs. However, this issue was partially addressed in recent work that builds on the findings of the current study using the same patient sample. Li Sin, Mainland, Lee, Ornstein, Shulman, and Herrmann (2015) hypothesized that CFs may contribute to higher morbidity and mortality rates in patients with dementia. To investigate this association, patients were followed for six months following their initial assessment. Ratings of CFs, collected during the initial assessment, were correlated with variables relating to hospitalizations or death. Four participants required hospitalization and four died during the follow-up period. Although the study was limited by the very small number of patients who experienced significant health-related events during the follow-up period, hospitalization and death was not found to be significantly associated with initial ratings of CFs. However, future research is needed to expand on this preliminary finding through use of longer follow-up periods and a larger sample. 
As mentioned previously, the interaction between cognitive-enhancing medications and CFs remains largely unexamined. There is some evidence to suggest that cholinesterase inhibitors are most effective in treating dementia when patients are presenting with fluctuating cognition (Onofrj et al., 2003); however, to date this association has only been examined in patients with very mild dementia and over a relatively short period of time (i.e., six months). Therefore, additional research is needed to determine whether this pattern holds true throughout the course of the disease and across different types of dementia.

Another intriguing avenue for future research in this area involves the use of neuroimaging techniques to better capture and characterize CFs. Recently, Franciotti et al. (2013) examined default mode network resting state activity in AD and DLB to determine if such activity was altered in patients with CFs. Although they found that functional connectivity was reduced in dementia patients, they found no particular association between CFs and the default mode network. Peraza et al. (2014) found similar results in a study comparing patients with DLB to healthy controls. However, in Peraza et al.'s study, the authors did find that desynchronization of a number of cortical and subcortical areas related to the left fronto-parietal network was associated with the severity and frequency of CFs. Thus, there appears to be empirical evidence for the potential role of attention-executive networks in the aetiology of CFs (Peraza et al., 2014). However, future research is needed to determine whether this pattern of neural disconnectivity plays a role in presence of CFs in other dementia types.

Finally, legal issues associated with CFs have become the focus of a small body of recent research that is in need of further investigation. The 'lucid interval' is a long held legal concept widely accepted in case law as a possible means of countering a challenge to testamentary and related capacities (Shulman et al., in press). Essentially, the term implies that patients with 
dementia have periodic intervals when their level of function returns to 'normal' or 'near normal' levels. Lawyers have long argued that, during these intervals, patients may possess adequate reasoning to make important legal decisions. Researchers have suggested that the legal concept of a lucid interval is perhaps best understood using the medical phenomenon of CFs (Shulman et al., in press). However, based on the limited medical research on the subject of CFs, the application of the lucid interval to dementia appears to be invalid. This invalidity is related to the fact that fluctuations are generally not so large as to render a previously incapable person to be temporarily able to execute a will (Shulman et al., in press.). The influence of CFs on decision making ability specifically related to medical treatments has also received recent attention (Trachsel, Hermann, \& Biller-Andorno, 2014). Thus, an interesting avenue for future research could involve a longitudinal study of decision-making ability in patients with dementia and whether CFs predict which patients will be deemed incompetent in terms of their decisionmaking capacity. 


\section{Conclusion}

The purpose of the current study was to investigate the nature of CFs in a sample of individuals with dementia living in a long-term care facility. Results of the current study suggest that CFs exert a broad range of influence over patients' functional abilities and wellbeing. Specifically, increasing severity of CFs is associated with lower global performance on cognitive measures. The specific profile of cognitive deficits associated with CFs differs depending on the method used to identify CFs, but a profile of reduced language, attention and memory appears to be most consistently related to CFs across the limited number of studies that have investigated this phenomenon. Importantly, nurse-based ratings of patients' ability to engage in activities of daily living and their general quality of life were significantly lower in patients with more severe CFs. The implication of this finding is of particular relevance when planning care for individuals with dementia, as the presence of CFs may represent a 'red flag' identifying patients in need of additional access to supervision and treatments targeting mood and behaviours. Finally, the severity of CFs appears strongly associated with levels of caregiver burden in nurses working in long-term care facilities. In addition, working with patients with severe CFs is associated with lower levels of job satisfaction among nursing staff. This finding may partially explain the high turn-over rates of nursing staff in inpatient dementia clinics. From an administrative perspective, identifying which patients experience CFs may help with staffing decisions and patient placement planning. Overall, the characterization of CFs remains a challenging task that is strongly influenced by the particular tool selected to classify them. However, identifying which patients experience CFs could play an important role in developing individualized treatment plans best suited for patients specific care needs. 


\section{References}

Alzheimer Society of Canada. (2010). Rising tide: The impact of dementia on Canadian society. Ottawa, ON: Author.

American Psychiatric Association. (2000). Diagnostic and Statistical Manual of Mental Disorders, Fourth Edition: DSM-IV-TR. Washington, D. C.: American Psychiatric Association.

Ardila, A., Ostrosky-Solis, F., Rosselli, M., \& Gómez, C. (2000). Age-Related Cognitive Decline During Normal Aging: The Complex Effect of Education. Archives of Clinical Neuropsychology, 15(6), 495-513. http://doi.org/10.1016/S0887-6177(99)00040-2

Ballard, C. G., Aarsland, D., McKeith, I., O’Brien, J., Gray, A., Cormack, F., ... Tovee, M. (2002). Fluctuations in attention: PD dementia vs DLB with parkinsonism. Neurology, 59(11), 1714-1720. doi:10.1212/01.WNL.0000036908.39696.FD

Ballard, C., O’Brien, J., Gray, A., \& Cormack, F. (2001). Attention and fluctuating attention in patients with dementia with Lewy bodies and alzheimer disease. Archives of Neurology, 58(6), 977. doi:10.1001/archneur.58.6.977

Ballard, C., Walker, M., O’Brien, J., \& Rowan, E. (2001). The characterisation and impact of "fluctuating" cognition in dementia with Lewy bodies and Alzheimer's disease. International Journal of Geriatric Psychiatry, 16(5), 494-498. doi:10.1002/gps.368

Beck, C., Ortigara, A., Mercer, S., \& Shue, V. (1999). Enabling and empowering certified nursing assistants for quality dementia care. International Journal of Geriatric Psychiatry, 14(3), 197-211. doi:10.1002/(SICI)1099-1166(199903)14:3<197::AIDGPS972>3.0.CO;2-Q 
Beglinger, L. J., Gaydos, B., Tangphao-Daniels, O., Duff, K., Kareken, D. A., Crawford, J., ... Siemers, E. R. (2005). Practice effects and the use of alternate forms in serial neuropsychological testing. Archives of Clinical Neuropsychology, 20(4), 517-529. http://doi.org/10.1016/j.acn.2004.12.003

Binder, E. F., Storandt, M., \& Birge, S. J. (1999). The relation between psychometric test performance and physical performance in older adults. The Journals of Gerontology. Series A, Biological Sciences and Medical Sciences, 54(8), M428-432. doi:10.1093 /gerona/54.8.M428

Boström, F., Jonsson, L., Minthon, L., \& Londos, E. (2007). Patients with dementia with lewy bodies have more impaired quality of life than patients with Alzheimer disease. Alzheimer Disease and Associated Disorders, 21, 150-154. doi: 10.1186/alzrt251

Boström, F., Jönsson, L., Minthon, L., \& Londos, E. (2007). Patients with Lewy body dementia use more resources than those with Alzheimer's disease. International Journal of Geriatric Psychiatry, 22(8), 713-719. http://doi.org/10.1002/gps.1738

Bradshaw, J., Saling, M., Hopwood, M., Anderson, V., \& Brodtmann, A. (2004). Fluctuating cognition in dementia with Lewy bodies and Alzheimer's disease is qualitatively distinct. Journal of Neurology, Neurosurgery, and Psychiatry, 75(3), 382-387. http://doi.org/10.1136/jnnp.2002.002576

Briel, R., McKeith, I., Barker, W., \& Hewitt, Y. (1999). EEG findings in dementia with Lewy bodies and Alzheimer's disease. Journal of Neurology, Neurosurgery, and Psychiatry, 66(3), 401-403. doi:10.1136/jnnp.66.3.401 
Buck, K. K., Atkinson, T. M., \& Ryan, J. P. (2008). Evidence of practice effects in variants of the Trail Making Test during serial assessment. Journal of Clinical and Experimental Neuropsychology, 30(3), 312-318. http://doi.org/10.1080/ 13803390701390483

Buckner, L., \& Yeandle, S. (2011). Valuing Carers: Calculating the Value of Unpaid Care. London, UK: Carers UK.

Charlson, M. E., Pompei, P., Ales, K. L., \& MacKenzie, C. R. (1987). A new method of classifying prognostic comorbidity in longitudinal studies: Development and validation. Journal of Chronic Diseases, 40(5), 373-383. http://doi.org/10.1016/0021$9681(87) 90171-8$

Chew, M. L., Mulsant, B. H., Pollock, B. G., Lehman, M. E., Greenspan, A., Mahmoud, R. A., ... Gharabawi, G. (2008). Anticholinergic Activity of 107 Medications Commonly Used by Older Adults. Journal of the American Geriatrics Society, 56(7), 1333-1341. http://doi.org/10.1111/j.1532-5415.2008.01737.x

Chu, C. H., Wodchis, W. P., \& McGilton, K. S. (2013). Turnover of regulated nurses in longterm care facilities. Journal of Nursing Management, 22(5), 553-562. http://doi.org /10.1111/jonm.12031

Cohen, J., Cohen, P., West, S. G., \& Aiken, L. S. (2002). Applied Multiple Regression/Correlation Analysis for the Behavioral Sciences, 3rd Edition (Third edition). Mahwah, N.J: Routledge.

Collie, A., Maruff, P., Darby, D. G., \& McStephen, M. (2003). The effects of practice on the cognitive test performance of neurologically normal individuals assessed at brief testretest intervals. Journal of the International Neuropsychological Society: JINS, 9(3), 419-428. http://doi.org/10.1017/S1355617703930074 
Cummings, J. L., Mega, M., Gray, K., Rosenberg-Thompson, S., Carusi, D. A., \& Gornbein, J. (1994). The Neuropsychiatric Inventory: comprehensive assessment of psychopathology in dementia. Neurology, 44(12), 2308-2314. doi:10.1212/ WNL.44.12.2308

DeVellis, R. F. (2003). Scale Development: Theory and Applications. CA, USA: Sage Publication.

Escandon, A., Al-Hammadi, N., \& Galvin, J. E. (2010). Effect of cognitive fluctuation on neuropsychological performance in aging and dementia. Neurology, 74(3), 210-217. http://doi.org/10.1212/WNL.0b013e3181ca017d

Fastenau, P. S., Hankins, W. T., McGinnis, C. M., Moy, T., \& Richard, M. (2002). Effects of alternate forms on retest effects in clinical test [abstract]. Journal of the International Neuropsychological Society, 7, 151.

Faul, F., Erdfelder, E., Buchner, A., \& Lang, A.-G. (2009). Statistical power analyses using G*Power 3.1: tests for correlation and regression analyses. Behavior Research Methods, 41(4), 1149-1160. http://doi.org/10.3758/BRM.41.4.1149

Faul, F., Erdfelder, E., Lang, A.-G., \& Buchner, A. (2007). G*Power 3: a flexible statistical power analysis program for the social, behavioral, and biomedical sciences. Behavior Research Methods, 39(2), 175-191. doi:10.3758/BF03193146

Ferman, T. J., Smith, G. E., Boeve, B. F., \& Ivnik, R. J. (2004). DLB fluctuations: specific features that reliably differentiate DLB from AD and normal aging. Neurology, 62(2), 181. doi:10.1212/WNL.62.2.181

Ferris, S., Karantzoulis, S., Somogyi, M., \& Meng, X. (2013). Rivastigmine in moderately severe-to-severe Alzheimer's disease: Severe Impairment Battery factor analysis. Alzheimer's Research \& Therapy, 5(6), 63. http://doi.org/10.1186/alzrt229 
Field, A. (2005). Discovering Statistics Using SPSS (Second). CA, USA: Sage Publications Ltd. Folstein, M. F. (1997). Differential diagnosis of dementia: the clinical process. Psychiatric Clinics of North America, 20(1), 45-57. http://doi.org/10.1016/S0193-953X(05)70392-0

Folstein, M. F., Folstein, S. E., \& McHugh, P. R. (1975). "Mini-mental state". A practical method for grading the cognitive state of patients for the clinician. Journal of Psychiatric Research, 12(3), 189-198. doi:10.1016/0022-3956(75)90026-6

Franciotti, R., Falasca, N. W., Bonanni, L., Anzellotti, F., Maruotti, V., Comani, S., ... Onofrj, M. (2013). Default network is not hypoactive in dementia with fluctuating cognition: an Alzheimer disease/dementia with Lewy bodies comparison. Neurobiology of Aging, 34(4), 1148-1158. http://doi.org/10.1016/j.neurobiolaging. 2012.09.015

Ganguli, M., Ratcliff, G., Huff, J., Belle, S., Kancel, M. J., Fischer, L., ... Kuller, L. H. (1991). Effects of age, gender, and education on cognitive tests in a rural elderly community sample: norms from the Monongahela Valley Independent Elders Survey. Neuroepidemiology, 10(1), 42-52. http://doi.org/10.1159/000110246

Garbutt, S., Matlin, A., Hellmuth, J., Schenk, A. K., Johnson, J. K., Rosen, H., ... Boxer, A. L. (2008). Oculomotor function in frontotemporal lobar degeneration, related disorders and Alzheimer's disease. Brain, 131(5), 1268-1281. http://doi.org/ 10.1093/brain/awn047

Goldberg, T. E., Harvey, P. D., Wesnes, K. A., Snyder, P. J., \& Schneider, L. S. (2015). Practice effects due to serial cognitive assessment: implications for preclinical Alzheimer's disease randomized controlled trials. Alzheimer's \& Dementia: Diagnosis, Assessment \& Disease Monitoring, 1(1), 103-111. http://doi.org/ 10.1016/j.dadm.2014.11.003 
Hely, M. A., Reid, W. G. J., Halliday, G. M., \& McRitchie, D. A. (1996). Diffuse Lewy body disease: clinical features in nine cases without coexistent Alzheimer's disease. Journal of Neurology, Neurosurgery and Psychiatry, 60(5), 531-538. doi:10.1136/jnnp.60.5.531

Herrmann, N., Cappell, J., Eryavec, G. M., \& Lanctôt, K. L. (2011). Changes in nursing burden following memantine for agitation and aggression in long-term care residents with moderate to severe Alzheimer's disease: an open-label pilot study. CNS Drugs, 25(5), 425-433. http://doi.org/10.2165/11588160-000000000-00000

Hirst, M. (2005). Carer distress: A prospective, population-based study. Social Science \& Medicine, 61(3), 697-708. http://doi.org/10.1016/j.socscimed.2005.01.001

Howell, D. C. (2007). The analysis of missing data. In In Outhwaite, W. \& Turner, S. Handbook of Social Science Methodology. London, UK: Sage.

Imamura, T., Hirono, N., Hashimoto, M., Shimomura, T., Tanimukai, S., Kazui, H., ... Mori, E. (1999). Clinical diagnosis of dementia with Lewy bodies in a Japanese dementia registry. Dementia and Geriatric Cognitive Disorders, 10(3), 210-216. doi:10.1159/000017121

Inouye, S. K., van Dyck, C. H., Alessi, C. A., Balkin, S., Siegal, A. P., \& Horwitz, R. I. (1990). Clarifying confusion: the confusion assessment method. Annals of Internal Medicine, 113(12), 941 - 948. doi:10.7326/0003-4819-113-12-941

Jefferson, A. L., Paul, R. H., Ozonoff, A., \& Cohen, R. A. (2006). Evaluating elements of executive functioning as predictors of instrumental activities of daily living (IADLs). Archives of Clinical Neuropsychology: The Official Journal of the National Academy of Neuropsychologists, 21(4), 311-320. http://doi.org/10.1016/j.acn.2006.03.007

Jönsson, L., Andreasen, N., Kilander, L., Soininen, H., Waldemar, G., Nygaard, H., ... Wimo, A. (2006). Patient- and proxy-reported utility in Alzheimer disease using the EuroQoL. 
Alzheimer Disease and Associated Disorders, 20(1), 49-55. http://doi.org/ 10.1097/01.wad.0000201851.52707.c9

Jurica, P. J., Leitten, C. L., \& Mattis, S. (2001). Dementia Rating Scale-2 (DRS-2). Montreal: Psychological Research Institute.

Keating, N., \& Gaudet, N. (2012). Quality of life of persons with dementia. The Journal of Nutrition, Health \& Aging, 16(5), 454-456. http://doi.org/10.1007/s12603-011-0346-4

Kleinman, L., Frank, L., Ciesla, G., Rupnow, M., \& Brodaty, H. (2004). Psychometric performance of an assessment scale for strain in nursing care: The M-NCAS. Health and Quality of Life Outcomes, 2(1), 62-62. http://doi.org/10.1186/1477-7525-2-62

Kolbeinsson, H., \& Jónsson, A. (1993). Delirium and dementia in acute medical admissions of elderly patients in Iceland. Acta Psychiatrica Scandinavica, 87(2), 123-127.

Lee, D. R., McKeith, I., Mosimann, U., Ghosh-Nodial, A., Grayson, L., Wilson, B., \& Thomas, A. J. (2013). The Dementia Cognitive Fluctuation Scale, a new psychometric test for clinicians to identify cognitive fluctuations in people with dementia. The American Journal of Geriatric Psychiatry. http://doi.org/10.1016/j. jagp.2013.01.072

Lee, D. R., McKeith, I., Mosimann, U., \& Ghosh-Nodyal, A. (2013). Examining carer stress in dementia: the role of subtype diagnosis and neuropsychiatric symptoms. International Journal of Geriatric Psychiatry, 28(2), 135-141. http://doi.org/ 10.1002/gps.3799

Lee, D. R., Taylor, J.-P., \& Thomas, A. J. (2012). Assessment of cognitive fluctuation in dementia: a systematic review of the literature. International Journal of Geriatric Psychiatry, 27(10), 989-998. doi:10.1002/gps.2823 
Li Sin, G., Mainland, B. J., Lee, J., Ornstein, T. J., Shulman, K. I., \& Herrmann, N. (2015). Outcomes of cognitive fluctuations in dementia patients. Journal of the American Geriatrics Society, 63 (6), 1258-1260. 10.1111/jgs.13506

Little, R. J. (1988). A test of missing completely at random for multivariate data with missing values. Journal of the American Statistical Association, 83(404), 1198-1202. doi:10.1080/01621459.1988.10478722

Logsdon, R. G., Gibbons, L. E., McCurry, S. M., \& Teri, L. (2002). Assessing quality of life in older adults with cognitive impairment. Psychosomatic Medicine, 64(3), 510-519. doi:10.1097/00006842-200205000-00016

Luttenberger, K., Schmiedeberg, A., \& Gräßel, E. (2012). Activities of daily living in dementia: revalidation of the E-ADL test and suggestions for further development. BMC Psychiatry, 12(1), 208-218. http://doi.org/10.1186/1471-244X-12-208

MacKinnon, D. P., Krull, J. L., \& Lockwood, C. M. (2000). Equivalence of the mediation, confounding and suppression effect. Prevention Science : The Official Journal of the Society for Prevention Research, 1(4), 173. doi: 10.1023/A:1026595011371

Mahoney, E. K., Hurley, A. C., Volicer, L., Bell, M., Gianotis, P., Hartshorn, M., ... Novakoff, L. (1999). Development and testing of the Resistiveness to Care Scale. Research in Nursing \& Health, 22(1), 27-38. doi:10.1002/(SICI)1098-240X(199902)22:1<27::AIDNUR4>3.0.CO;2-T

Mahoney, P., Wood, V., \& Wade, D. (1965). Functional evaluation: The Barthel Index. Maryland State Medical Journal, 14, 61-65. doi:10.1161/01.str.30.8.1538

Mainland, B. J., \& Shulman, K. I. (2012). Clock Drawing Test. In Cognitive Screening Instruments: A Practical Approach (pp. 79-109). London, UK: Springer. 
Manero, R. M., Casals-Coll, M., Sánchez-Benavides, G., Rodríguez-de los Reyes, O. N., Aguilar, M., Badenes, D., ... Neuronorma Study Team. (2014). Diagnostic validity of the Alzheimer's disease functional assessment and change scale in mild cognitive impairment and mild to moderate Alzheimer's disease. Dementia and Geriatric Cognitive Disorders, 37(6), 366-375. http://doi.org/10.1159/000350800

McDonald, J. H. (2014). Handbook of Biological Statistics - Third Edition. Baltimore: Sparky House Publishing.

McKeith, I., Del Ser, T., Spano, P., Emre, M., Wesnes, K., Anand, R., ... Spiegel, R. (2000). Efficacy of rivastigmine in dementia with Lewy bodies: a randomised, double-blind, placebo-controlled international study. Lancet, 356(9247), 2031-2036. http://doi.org /10.1016/S0140-6736(00)03399-7

McKeith, I. G., Dickson, D. W., Lowe, J., Emre, M., O’Brien, J. T., Feldman, H., ... Consortium on DLB. (2005). Diagnosis and management of dementia with Lewy bodies: third report of the DLB Consortium. Neurology, 65(12), 1863-1872. http://doi.org/10.1212/01.wnl. 0000187889.17253.b1

McKeith, I. G., Galasko, D., Kosaka, K., Perry, E. K., Dickson, D. W., Hansen, L. A., ... Perry, R. H. (1996). Consensus guidelines for the clinical and pathologic diagnosis of dementia with Lewy bodies (DLB): report of the consortium on DLB international workshop. Neurology, 47(5), 1113-1124. doi:10.1212/WNL.47.5.1113

McKeith, I. G., Perry, R. H., Fairbairn, A. F., Jabeen, S., \& Perry, E. K. (1992). Operational criteria for senile dementia of Lewy body type (SDLT). Psychological Medicine, 22(4), 911-922. doi:10.1017/S0033291700038484 
Mega, M. S., Masterman, D. L., Benson, D. F., Vinters, H. V., Tomiyasu, U., Craig, A. H., ... Cummings, J. L. (1996). Dementia with Lewy bodies Reliability and validity of clinical and pathologic criteria. Neurology, 47(6), 1403-1409. http://doi.org/10.1212/ WNL.47.6. 1403

Mendez, M. F., Ala, T., \& Underwood, K. L. (1992). Development of scoring criteria for the clock drawing task in Alzheimer's disease. Journal of the American Geriatrics Society, 40 (11), 1095-1099. doi:10.1111/j.1532-5415.1992.tb01796.x

Mollenkopf, H., \& Walker, A. (2007). Quality of Life in Old Age: International and Multidisciplinary Perspectives. Dordrecht, The Netherlands: Springer.

Molyneux, G. J., McCarthy, G. M., McEniff, S., \& Cryan, M. (2008). Prevalence and predictors of carer burden and depression in carers of patients referred to an old age psychiatric service. International Psychogeriatrics, 20(6), 1193-1202. http://doi.org/10.1017/ S1041610208007515

Moore, A. R., \& O’Keeffe, D. S. T. (2012). Drug-induced cognitive impairment in the elderly. Drugs \& Aging, 15(1), 15-28. http://doi.org/10.2165/00002512-199915010-00002

Morris, J. N., Fries, B. E., Mehr, D. R., Hawes, C., Phillips, C., Mor, V., \& Lipsitz, L. A. (1994). MDS Cognitive Performance Scale@. Journal of Gerontology, 49(4), M174-M182. http://doi.org/10.1093/geronj/49.4.M174

Naglie, G., Tomlinson, G., Tansey, C., Irvine, J., Ritvo, P., Black, S. E., ... Krahn, M. (2006). Utility-based Quality of Life measures in Alzheimer's disease. Quality of Life Research: An International Journal of Quality of Life Aspects of Treatment, Care and Rehabilitation, 15(4), 631-643. http://doi.org/10.1007/s11136-005-4364-8 
National Collaborating Centre for Mental Health. (2007). Dementia: supporting people with dementia and their carers in health and social care. Great Britain: National Institute for Health and Clinical Excellence. Retrieved from https://www.nice.org.uk/guidance/cg42

Neil, W., \& Bowie, P. (2008). Carer burden in dementia--assessing the impact of behavioural and psychological symptoms via self-report questionnaire. International Journal of Geriatric Psychiatry, 23(1), 60-64. http://doi.org/10.1002/gps.1839

Onofri, E., Mercuri, M., Donato, G., \& Ricci, S. (2015). Cognitive fluctuations in connection to dysgraphia: a comparison of Alzheimer's disease with dementia Lewy bodies. Clinical Interventions in Aging, 625. http://doi.org/10.2147/CIA.S79679

Onofrj, M., Thomas, A., Iacono, D., Luciano, A. L., \& Di Iorio, A. (2003). The effects of a cholinesterase inhibitor are prominent in patients with fluctuating cognition: a part 3 study of the main mechanism of cholinesterase inhibitors in dementia. Clinical Neuropharmacology, 26(5), 239-251. doi: 10.1097/00002826-200309000-00008

Panisset, M., Roudier, M., Saxton, J., \& Boiler, F. (1994). Severe impairment battery: a neuropsychological test for severely demented patients. Archives of Neurology, 51(1), 41-45. doi:10.1001/archneur.1994.00540130067012

Pélissier, C., Roudier, M., \& Boller, F. (2001). Factorial validation of the Severe Impairment Battery for patients with Alzheimer's disease. A pilot study. Dementia and Geriatric Cognitive Disorders, 13(2), 95-100. doi: 10.1159/000048640

Peraza, L. R., Kaiser, M., Firbank, M., Graziadio, S., Bonanni, L., Onofrj, M., .. Taylor, J.-P. (2014). fMRI resting state networks and their association with cognitive fluctuations in dementia with Lewy bodies. NeuroImage. Clinical, 4, 558-565. http://doi.org/ 10.1016/j.nicl.2014.03.013 
Quan, H., Parsons, G. A., \& Ghali, W. A. (2002). Validity of information on comorbidity derived rom ICD-9-CCM administrative data. Medical Care, 40(8), 675-685. http://doi.org/ 10.1097/01.MLR.0000020927.46398.5D

Quan, H., Sundararajan, V., Halfon, P., Fong, A., Burnand, B., Luthi, J.-C., ... Ghali, W. A. (2005). Coding algorithms for defining comorbidities in ICD-9-CM and ICD-10 administrative data. Medical Care, 43(11), 1130-1139. doi:10.1097/01.mlr. 0000182534.19832 .83

Reed, G. F., Lynn, F., \& Meade, B. D. (2002). Use of coefficient of variation in assessing variability of quantitative assays. Clinical and Diagnostic Laboratory Immunology, 9(6), 1235-1239. http://doi.org/10.1128/CDLI.9.6.1235-1239.2002

Reitan, R. M., \& Wolfson, D. (1985). The Halstead-Reitan Neuropsychological Test Battery: Therapy and Clinical Interpretation. Tuscan, AZ: Neuropsychological Press.

Robert, P., Ferris, S., Gauthier, S., Ihl, R., Winblad, B., \& Tennigkeit, F. (2010). Review of Alzheimer's disease scales: is there a need for a new multi-domain scale for therapy evaluation in medical practice? Alzheimer's Research \& Therapy, 2(4), 24. http://doi.org/10.1186/alzrt48

Robertsson, B., Blennow, K., Gottfries, C. G., \& Wallin, A. (1998). Delirium in dementia. International Journal of Geriatric Psychiatry, 13(1), 49-56. doi:10.1002/(SICI)10991166(199801)13:1<49::AID-GPS733>3.0.CO;2-4

Rongve, A., Brønnick, K., Ballard, C., \& Aarsland, D. (2010). Core and suggestive symptoms of dementia with lewy bodies cluster in persons with mild dementia. Dementia and Geriatric Cognitive Disorders, 29(4), 317. doi:10.1159/000295111 
Sands, L. P., Ferreira, P., Stewart, A. L., Brod, M., \& Yaffe, K. (2004). What explains differences between dementia patients' and their caregivers' ratings of patients' quality of life? The American Journal of Geriatric Psychiatry: Official Journal of the American Association for Geriatric Psychiatry, 12(3), 272-280. doi:10.1097/00019442-20040500000006

Saxton, J., McGonigle-Gibson, K. L., Swihart, A. A., \& Miller, V. J. (1990). Assessment of the severely impaired patient: Description and validation of a new neuropsychological test battery. Psychological Assessment, 2(3), 298-303. doi:10.1037/1040-3590.2.3.298

Schafer, J. L. (1997). Analysis of Incomplete Multivariate Data (1 edition). London; New York: Chapman and Hall/CRC.

Schafer, J. L., \& Olsen, M. K. (1998). Multiple imputation for multivariate missing-data problems: a data analyst's perspective. Multivariate Behavioral Research, 33, 545-571. doi:10.1207/s15327906mbr3304_5

Schlomer, G. L., Bauman, S., \& Card, N. A. (2010). Best practices for missing data management in counseling psychology. Journal of Counseling Psychology, 57(1), 1-10. http://doi. $\operatorname{org} / 10.1037 / \mathrm{a} 0018082$

Schmidt, J. A., Wagner, C. C., \& Kiesler, D. J. (1999). Psychometric and circumplex properties of the octant scale Impact Message Inventory (IMI-C): A structural evaluation. Journal of Counseling Psychology, 46(3), 325-334. doi:10.1037/0022-0167.46.3.325

Schmitt, F. A., Ashford, W., Ernesto, C., Saxton, J., Schneider, L. S., Clark, C. M., ... Thal, L. J. (1997). The severe impairment battery: concurrent validity and the assessment of longitudinal change in Alzheimer's disease. Alzheimer Disease \& Associated Disorders, 11, 51-56. doi:10.1097/00002093-199700112-00008 
Serrano, C., \& García-Borreguero, D. (2004). Fluctuations in cognition and alertness in Parkinson's disease and dementia. Neurology, 63(8), S31-S34. doi:10.1212/ WNL.63.8_suppl_3.S31

Shulman, K. I., Hull, I. M., DeKoven, S., Amodeo, S., Mainland, B. J., \& Herrmann, N. (In press.). Cognitive fluctuations and the lucid interval in dementia: Medio-legal implications for testamentary capacity. Journal of the American Academy of Psychiatry and the Law.

Shuster Jr, J. L. (2000). Palliative care for advanced dementia. Clinics in Geriatric Medicine, 16(2), 373-386. http://doi.org/10.1016/S0749-0690(05)70062-8

Sikkes, S. A. M., de Lange-de Klerk, E. S. M., Pijnenburg, Y. A. L., \& Gillissen, F. (2012). A new informant-based questionnaire for instrumental activities of daily living in dementia. Alzheimer's \& Dementia: The Journal of the Alzheimer's Association, 8(6), 536-543. doi:10.1016/j.jalz.2011.08.006

Simpson, P. M., Surmon, D. J., Wesnes, K. A., \& Wilcock, G. K. (1991). The cognitive drug research computerized assessment system for demented patients: A validation study. International Journal of Geriatric Psychiatry, 6(2), 95-102. http://doi.org/10.1002/ gps.930060208

Statistics Canada. (2010). An aging population. Retrieved from http://www.statcan.gc.ca/pub/11402-x/2010000/chap/pop/pop02-eng.htm

Stevens, J. (2002). Applied Multivariate Statistics for the Social Sciences. Mahjah, NJ: Lawrence Erlbaum Associates.

Tabachnik, B. G., \& Fidell, L. S. (2001). Using multivariate statistics (4th ed.). Boston, MA: Allyn \& Bacon. 
Tartaglia, M. C., Zhang, Y., Racine, C., Laluz, V., Neuhaus, J., Chao, L., ... Weiner, M. (2011). Executive dysfunction in frontotemporal dementia is related to abnormalities in frontal white matter tracts. Journal of Neurology, 259(6), 1071-1080. http://doi.org/10.1007/ s00415-011-6300-x

Tavakol, M., \& Dennick, R. (2011). Making sense of Cronbach's alpha. International Journal of Medical Education, 2, 53-55. http://doi.org/10.5116/ijme.4dfb.8dfd

The EuroQol Group. (1990). EuroQol--a new facility for the measurement of health-related quality of life. The EuroQol Group. Health Policy (Amsterdam, Netherlands), 16(3), 199-208. doi: 10.1016/0168-8510(90)90421-9

Trachsel, M., Hermann, H., \& Biller-Andorno, N. (2014). Cognitive Fluctuations as a Challenge for the Assessment of Decision-Making Capacity in Patients With Dementia. American Journal of Alzheimer's Disease and Other Dementias. http://doi.org/10.1177/ 1533317514539377

Varanese, S. (2010). Fluctuating cognition and different cognitive and behavioural profiles in Parkinson's disease with dementia: comparison of dementia with Lewy bodies and Alzheimer's disease. Journal of Neurology, 257(6), 1004-1011. doi:10.1007/s00415-010$5453-3$

Wagner, M. T., \& Bachman, D. L. (1996). Neuropsychological features of diffuse Lewy body disease. Archives of Clinical Neuropsychology, 11(3), 175-184. http://doi.org/10.1016/ 0887-6177(95)00022-4

Walker, E. A., Unutzer, J., Rutter, C., Gelfand, A., Saunders, K., VonKorff, M., ... Katon, W. (1999). Costs of health care use by women HMO members with a history of childhood 
abuse and neglect. Archives of General Psychiatry, 56(7), 609. doi:10.1001/ $\operatorname{archpsyc.56.7.609}$

Walker, M. P., Ayre, G. A., Perry, E. K., Wesnes, K., McKeith, I. G., Tovee, M., ... Ballard, C. G. (2000). Quantification and Characterisation of Fluctuating Cognition in Dementia with Lewy Bodies and Alzheimer\&rsquo;s Disease. Dementia and Geriatric Cognitive Disorders, 11(6), 327-335. http://doi.org/10.1159/000017262

Walker, M. P., Ballard, C. G., Ayre, G. A., Wesnes, K., Cummings, J. L., McKeith, I. G., \& O’Brien, J. (2000). The Clinician Assessment of Fluctuation and the One Day Fluctuation Assessment Scale Two methods to assess fluctuating confusion in dementia. The British Journal of Psychiatry, 177(3), 252-256. doi:10.1192/bjp.177.3.252

Wechsler, D. (1993). WAIS-III/WMS-III Technical Manual. San Antonio, TX: The Psychological Corporation.

Wechsler, D. (2008). Wechsler Adult Intelligence Scale - Fourth Edition. San Antonio, TX: Pearson Assessment.

Weiner, M. F., Martin-Cook, K., Svetlik, D. A., Saine, K., Foster, B., \& Fontaine, C. S. (2000). The quality of life in late-stage dementia (QUALID) scale. Journal of the American Medical Directors Association, 1(3), 114-116. doi:10.4172/2161-0460.1000104

Weintraub, S., Baratz, R., \& Mesulam, M.-M. (1982). Daily living activities in the assessment of dementia. In Alzheimer's disease: a report of progress in research, edited by Corkin S, Davis K, Crowden J, et al. (pp. 189-192). NY, USA: Raven.

Wilson, R. S., McCann, J. J., Li, Y., Aggarwal, N. T., Gilley, D. W., \& Evans, D. A. (2007). Nursing home placement, day care use, and cognitive decline in Alzheimer's disease. The American Journal of Psychiatry, 164(6), 910-915. doi: 10.1176/appi.ajp.164.6.910 
World Health Organization. (1992). International Statistical Classification of Diseases and Related Health Problems, 10th Revision (ICD-10). Geneva: World Health Organization.

Zgaljardic, D. J., \& Benedict, R. H. (2001). Evaluation of practice effects in language and spatial processing test performance. Applied Neuropsychology, 8(4), 218-223. http://doi.org/ 10.1207/S15324826AN0804_4 